\title{
Nonergodic Delocalized States for Efficient Population Transfer within a Narrow Band of the Energy Landscape
}

\author{
Vadim N. Smelyanskiy $\odot,{ }^{1, *}$ Kostyantyn Kechedzhi, ${ }^{1,2,3}$ Sergio Boixo, ${ }^{1}$ \\ Sergei V. Isakov, ${ }^{4}$ Hartmut Neven, ${ }^{1}$ and Boris Altshuler ${ }^{5}$ \\ ${ }^{1}$ Google, Venice, California 90291, USA \\ ${ }^{2}$ QuAIL, NASA Ames Research Center, Moffett Field, California 94035, USA \\ ${ }^{3}$ University Space Research Association, 615 National Avenue, Mountain View, California 94043, USA \\ ${ }^{4}$ Google, 8002 Zurich, Switzerland \\ ${ }^{5}$ Physics Department, Columbia University, 538 West 120th Street, New York, New York 10027, USA
}

(Received 28 May 2018; revised manuscript received 20 August 2019; published 24 January 2020)

We address the long-standing problem of the structure of the low-energy eigenstates and long-time coherent dynamics in quantum spin-glass models. Below the spin-glass freezing transition, the energy landscape of the spin system is characterized by a proliferation of local minima where classical dynamics gets trapped. A theoretical description of quantum dynamics in this regime is challenging due to the complex nature of the distribution of the tunneling matrix elements between the local minima of the energy landscape. We study the transverse-field-induced quantum dynamics of the following "impurity band" (IB) spin model: zero energy of all spin configurations except for a small fraction of spin configurations ("marked states") that form a narrow band at a large negative energy. At a zero transverse field, the IB model demonstrates the freezing transition at inverse temperature $\beta_{f} \sim 1$ characterized by a nonzero value of the Edwards-Anderson order parameter. At a finite transverse field, the low-energy dynamics can be described by the effective downfolded Hamiltonian that acts in the Hilbert subspace involving only the marked states. We obtain in an explicit form the heavy-tailed probability distribution of the off-diagonal matrix elements of the down-folded Hamiltonian. This Hamiltonian is dense and belongs to the class of preferred basis Levy matrices. Analytically solving nonlinear cavity equations for the ensemble of down-folded Hamiltonians allows us to describe the statistical properties of the eigenstates. In a broad interval of transverse fields, they are nonergodic, albeit extended. It means that the band of marked states splits into a set of narrow minibands. Accordingly, the quantum evolution that starts from a particular marked state leads to a linear combination of the states belonging to a particular miniband. An analytical description of this qualitatively new type of quantum dynamics is a key result of our paper. Based on our analysis, we propose the population transfer (PT) algorithm: The quantum evolution under constant transverse field $B_{\perp}$ starts at a low-energy spin configuration and ends up in a superposition of $\Omega$ spin configurations inside a narrow energy window. This algorithm crucially relies on the nonergodic nature of delocalized low-energy eigenstates. In the considered model, the run-time of the best classical algorithm (exhaustive search) is $t_{\mathrm{cl}}=2^{n} / \Omega$. For $\sqrt{n} \gg B_{\perp} \gg 1$, the typical runtime of the quantum PT algorithm $\sqrt{t_{\mathrm{cl}}} e^{n /\left(2 B_{\perp}^{2}\right)}$ scales with $n$ and $\Omega$ as that of Grover's quantum search, except for the small correction to the exponent. Unlike the Hamiltonians proposed for analog quantum unstructured search algorithms, the model we consider is nonintegrable and the transverse field delocalizes the marked states. As a result, our PT protocol does not require fine-tuning of the transverse field and may be initialized in a computational basis state. We find that the run-times of the PT algorithm are distributed according to the alpha-stable Levy law with tail index 1. We argue that our approach can be applied to study the PT protocol in other transverse-field spin-glass models, with a potential quantum advantage over classical algorithms.

DOI: 10.1103/PhysRevX.10.011017

Subject Areas: Condensed Matter Physics,

Quantum Physics, Quantum Information

\footnotetext{
*Corresponding author. smelyan@google.com

Published by the American Physical Society under the terms of the Creative Commons Attribution 4.0 International license. Further distribution of this work must maintain attribution to the author(s) and the published article's title, journal citation, and DOI.
}

\section{INTRODUCTION}

The idea to use quantum computers for the solution of search and discrete optimization problems has been actively pursued for decades, most notably in connection to Grover's algorithm [1], quantum annealing [2-10], and, more recently, quantum approximate optimization [11]. 
Hard optimization problems have their counterparts in spinglass models of statistical physics $[12,13]$. The energy function of a hard optimization problem is characterized by a large number of spurious local minima separated from each other by a large Hamming distance (number of bit flips transforming one to another). At a low temperature, the rate of transition between the local minima is exponentially suppressed, and, therefore, the classical dynamics (realized, for example, via the single-spin-flip Metropolis algorithm) remains constrained to the vicinity of a single local minimum and is, therefore, inefficient at exploring the configuration space, i.e., is nonergodic. Consider an application to such a nonergodic classical regime of a quantum computer (QC) realized using a system of interacting qubits (quantum spins $1 / 2$ ) in a transverse field:

$$
\begin{gathered}
H=H_{\mathrm{cl}}+H_{D}, \quad H_{D}=-B_{\perp} \sum_{k=0}^{n} \sigma_{x}^{k}, \\
H_{\mathrm{cl}}=\sum_{z} \mathcal{E}(z)|z\rangle\langle z|,
\end{gathered}
$$

where the energy function $\mathcal{E}(z)$ is defined over the set of $2^{n}$ configurations of $n$ bits (bit strings) $z=\left(z^{1}, z^{2}, \ldots, z^{n}\right)$, where $z^{k}=\{0,1\}$, computational basis, and $\sigma_{\alpha}^{k}, \alpha=x, y, z$, are Pauli matrices. Off-diagonal matrix elements connect states separated by one bit flip; therefore, Eq. (1) describes the Hamiltonian of the tight-binding model with diagonal disorder on the Boolean hypercube [14]. It is a generalization of the Anderson model initially introduced in the context of transport in finite-dimensional lattices [15-17].

An important insight into the output of quantum operations realized in the model Eq. (2) is provided by the structure of its eigenstates in the computation basis which also fully determines the quantum dynamics in the model. For a sufficiently weak transverse field, the eigenstates are localized in the space of bit strings; i.e., each one has significant amplitudes on a few bit strings which form its support set. An increasing transverse field results in the amplitude spreading over a subset of bit strings which increases exponentially with the number of qubits $n$. Such eigenstates are called extended. The transition between the extended and localized phases of a QC is an example of the many-body localization (MBL) transition, recently identified in models of mean-field spin glass $[5,18,19]$, with important implications for the efficiency of the quantum annealing algorithm $[7,14,20]$.

At a sufficiently strong transverse field, eigenstates could be extended and ergodic (EE), meaning that their amplitudes are spread over the entire Hilbert space, and probabilities to detect any particular bit string is of the order of the inverse number of bit strings $N^{-1}=2^{-n}$. EE states arise, for example, as a result of applying a random unitary to a product state or as eigenstates of a random Hamiltonian drawn from a Gaussian random matrix ensemble. However, in general, the extended state could be spread over a much smaller number of bit strings $N_{S} \propto N^{D}=2^{n D}$. The corresponding exponent $D$ is the fractal dimension of the eigenstate's support set. Localized states correspond to $D=0$ and EE to $D=1$, and the case $0<D<1$ we call nonergodic extended (NEE).

NEE eigenstates have been discovered in random matrix models such as the Rosenzweig-Porter model [21]. It has been proposed that Anderson models on random regular graphs [22] also demonstrate a NEE phase; however, this question remains open and an active topic of research [23-25]. In this paper, we demonstrate that the existence of NEEs is not limited to abstract matrix models. On the contrary, we show conclusively that NEEs arise naturally in a broad class of quantum spin-glass Hamiltonians of the form Eq. (2). A transverse field applied to a nonergodic classical model in Eq. (1) gives rise to tunneling matrix elements between its deep local minima. In this regime, NEE eigenstates could be formed by coherent superpositions of local minima separated by large Hamming distances. Such coherent superpositions form minibands of states with shared support sets.

A theoretical description of such nonergodic extended states for quantum spin-glass models is very challenging. A key challenge is the calculation of the statistics of the tunneling matrix elements between deep local minima separated by large Hamming distances $d$. On the one hand, the many-body delocalized regime is associated with the divergence of the so-called locator expansion [16], which is related to the transverse-field perturbation theory in the case of Eq. (1), which, therefore, is no longer a well-controlled expansion for the tunneling matrix element. Moreover, in general, the tunneling matrix element is given by a sum over a large number of virtual trajectories which interfere with each other, which is the case for the model considered in this paper as we show below. As a result, the perturbative expansion of the matrix element in $B_{\perp}$ must include multiple looped trajectories and, therefore, cannot be approximated by the leading-order term, also called the forward scattering approximation (FSA) [26]. On the other hand, a direct numerical solution of the Schrödinger equation is possible only for a very small system size, and, therefore, the results are strongly affected by finite size effects and are inconclusive in the NEE regime. Therefore, up to this point, there is no well-controlled theory of a NEE phase in a quantum spin glass.

A key technical achievement of this paper is the novel theoretical approach that allows an asymptotically exact analytical calculation of the matrix elements and their statistics as well as a well-controlled description of the structure of NEE eigenstates and the associated quantum dynamics. We apply our approach to a sufficiently simple yet nontrivial impurity band model of $\mathcal{E}(z)$ :

$$
H_{\mathrm{cl}}=\sum_{j=1}^{M} \mathcal{E}\left(z_{j}\right)\left|z_{j}\right\rangle\left\langle z_{j}\right| .
$$


Here, $M \gg 1$ marked states $\left|z_{j}\right\rangle$ ( $n$-bit strings $z_{j}$ ) are chosen uniformly at random from all bit strings of length $n$, with energies $\mathcal{E}\left(z_{j}\right)$ independently distributed around $-n$ within a narrow band of width $W \ll B_{\perp}$. All other states $z$ have energies $\mathcal{E}(z)=0$ and are separated by a large gap $\simeq n$ from the very narrow band of marked states (see Fig. 1). This model is inspired by the impurity band model in doped semiconductors [31]. It also corresponds to a classical unstructured search problem with multiple marked states. Each marked state $\mathcal{E}(z)$ is a deep local minimum of the classical energy landscape: A single spin flip with high probability raises the energy to $\mathcal{E}(z)=0$ and is, therefore, strongly suppressed. For $M \ll 2^{n}$, randomly chosen marked states are separated by Hamming distances that scale with $n$. A classical IB model at low temperature $\beta>$ $\beta_{f}=\ln 2-(1 / n) \ln M$ captures the frozen phase of a spin glass at the tail of the density of states, which, as we show below, is characterized by a nonzero Edwards-Anderson order parameter.

We develop a microscopic analytical theory of the "minibands" of nonergodic delocalized states in the IB model. We derive an effective down-folded Hamiltonian in the energy strip associated with the marked states. Its matrix elements correspond to the tunneling between the deep local minima described by the Wentzel-Kramers-Brillouin (WKB)

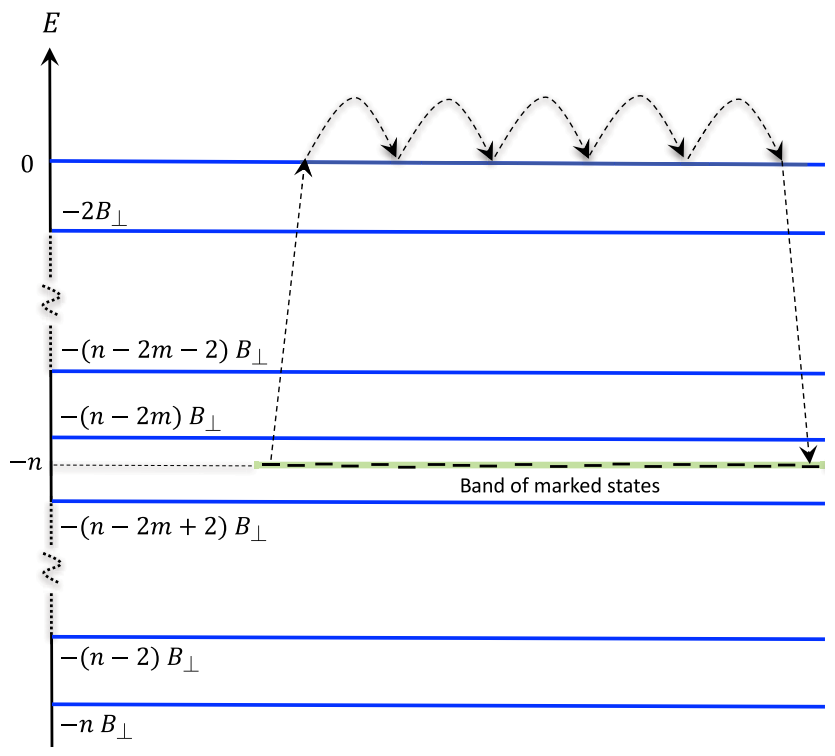

FIG. 1. Cartoon of the level diagram. Horizontal blue lines depict the energy levels $-B_{\perp}(n-2 m)$ of the driver Hamiltonian $H_{D}$ in Eq. (1) separated by $2 B_{\perp}$. A narrow impurity band of width $W \ll B_{\perp}$ is marked in light green. The sequence of short black lines depicts the energies of marked states $\mathcal{E}\left(z_{i}\right)$. Dashed lines depict the elementary path to the leading-order perturbation theory in $B_{\perp}$ for the tunneling matrix element $c_{i j}(E)$ given in Eq. (19). In this paper, we focus on the case of relatively large transverse fields $B_{\perp}>1$ so that the IB energies lie above the ground state of the total Hamiltonian (1) that corresponds to nearly all qubits polarized in the $x$ direction. analysis. Remarkably, the distribution of the matrix elements is heavy tailed. The ensemble of down-folded Hamiltonians for the marked state subspace corresponds to the preferred basis Levi matrices. We use the cavity method for Levi matrices [32-37] to find analytically the fractal dimension of the delocalized minibands and the probability distribution of their spectral width. This method also determines the probability distribution and the scaling with $n$ of the set of characteristic timescales that describe the quantum dynamics of the NEE minibands.

Our asymptotically exact analysis gives a number of predictions that cannot be reproduced within the leadingorder perturbation theory in a transverse field, called the forward scattering approximation (FSA) in the literature. Most notably, we uncover an asymptotic orthogonality of the subspace of marked states to the rest of the Hilbert space that extends, rather counterintuitively, to large transverse-field regime $B_{\perp} \gg 1$. As shown in detail below, accounting for this orthogonality catastrophe is critically important for the correct calculation of fractal dimensions of the support set and the miniband width statistics in the spectrum and, therefore, for the prediction for quantum dynamics of the IB model Eq. (3). In other words, the FSA gives a qualitatively incorrect prediction for the quantum dynamics of the NEE phase of the IB.

It is crucial that the dynamics within the IB of model (3) in the transverse field can be nonergodic yet delocalized in the computational basis. The model is by no means unique from this point of view. We believe that extended but nonergodic quantum states exist for quantum extensions of any problem Hamiltonian which is characterized by a classical spin-glass behavior: for the random energy model [30], Sherrington-Kirkpatrick model [38], $p$-spin model [39], $K$ satisfiability [40], etc.

Indeed, the main difference between classical and quantum spin-glass models is the existence of the MBL phase in the latter case. However, we see no reason to expect a direct transition between the MBL and ergodic phases without an intermediate nonergodic phase similar to the case of ordinary Anderson localization in finitedimensional space. This difference is due to the fact that the number of relevant bit strings at a given Hamming distance $d$ from a given one increases for spin-glass models exponentially with $d$, or even quicker, whereas for finitedimensional models this increase is only polynomial.

The formation of minibands of NEE eigenstates has important implications for quantum search and optimization algorithms. Quantum tunneling of collective spin excitations was proposed and studied experimentally as a mechanism for moving between states in the energy landscape that can lead to shorter transition timescales compared to classical simulated annealing approaches under certain conditions [4]. Experimental evidence of the faster timescales was later corroborated numerically using an imaginary-time quantum Monte Carlo (QMC) 
algorithm [41,42]. Furthermore, recent studies [43,44] show that, in QMC, the tunneling corresponds to the Kramers escape through the free-energy barrier in an extended spin system that includes spin replicas in an imaginary time direction. As a result, the incoherent quantum tunneling rate does not have a scaling advantage over such a QMC simulation. This result happens because incoherent tunneling dynamics corresponded to sequential transitions connecting individual minima, where each transition is dominated by a single tunneling path [43]. NEE eigenstates give rise to a qualitatively different tunneling dynamics, where a large number of tunneling paths interfere constructively, giving rise to minibands of the nonergodic many-body states delocalized in the computational basis. We demonstrate that transport within the minibands can be used for an efficient quantum search in spin-glass problems and propose a population transfer (PT) protocol based on this theoretical insight. In the case of a search in the space of marked states in the IB model, PT asymptotically approaches the run-time of the multitarget Grover which is optimal for unstructured problems such as IB.

The paper is organized as follows. Section II contains an analysis of the classical IB model, a qualitative discussion of the main results, and a detailed description of the population transfer protocol and its run-time. In Sec. III, we develop a down-folding procedure to reduce the original problem to the nonlinear eigenproblem in the marked state subspace. In Sec. IV, we calculate the off-diagonal (tunneling) matrix elements of the down-folded Hamiltonian and studied their dependence on $n$ and the Hamming distance using the WKB theory. In Sec. V, we develop an expansion of the nonlinear eigenproblem near the center of the IB shifted by a transverse field and obtain the effective Hamiltonian $\mathcal{H}$ of the PT problem. In Sec. VI, we study the statistical ensemble of Hamiltonians $\mathcal{H}$. Section VII discusses numerical results. In Sec. VIII, we study the PT within the Born approximation. In Sec. IX, we estimate the number of states in the miniband. In Sec. X, we provide an overview of the cavity method for dense random matrices. In Sec. XI, we solve the cavity equations and obtain the distributions of the real and imaginary parts of self-energy. In Sec. XII, we discuss the complexity of the PT algorithm. In Sec. XIII, we provide a comparison between PT and Grover's algorithm with multiple target states and systematic errors in the oracle phase and driver weight. In Sec. XIV, we provide a summary and concluding remarks.

\section{BACKGROUND AND QUALITATIVE SUMMARY OF RESULTS}

\section{A. Impurity band model: Classical spin-glass freezing transition}

It is instructive to discuss spin-glass characteristics of the classical IB model $H_{\mathrm{cl}}$ in Eq. (3). Its free energy demonstrates a discontinuity:

$$
f \approx\left[\begin{array}{ll}
-\frac{1}{\beta} \ln 2, & \beta<\beta_{f}, \\
-\frac{1}{\beta} \ln M-1, & \beta>\beta_{f},
\end{array}\right.
$$

at the inverse temperature of the freezing transition $\beta_{f}=$ $\ln 2-(1 / n) \ln M$ which separates the high-temperature regime $\beta<\beta_{f}$ with the statistical weight concentrated on $2^{n}-M$ states with $\mathcal{E}(z)=0$ and the low-temperature regime $\beta>\beta_{f}$ with the statistical weight concentrated in the band of marked states $-n+W \leq \mathcal{E}(z) \leq-n-W$. Below the transition temperature for any of the marked states, the rate of a single bit flip is exponentially small $\exp [-\beta|\mathcal{E}(z)|]$, and, therefore, the classical single-spin-flip dynamics of the model is frozen. Such a nonergodic classical dynamical phase is characterized by a nonzero value of the Edwards-Anderson order parameter [45]:

$$
q_{\mathrm{EA}} \equiv \lim _{t \rightarrow \infty} \lim _{n \rightarrow \infty}\left\{\left[s_{i}^{z}\left(t_{0}+t\right) s_{i}^{z}\left(t_{0}\right)\right]_{\mathrm{th}}\right\}_{\mathrm{dis}},
$$

where $s_{i}^{z}(t)$ stands for the value of the projection the $i$ th spin on the $z$ axis at time $t$ resulting from the singlespin-flip dynamics. In Eq. (5), $[\ldots]_{\text {th }}$ stands for averaging over the thermal ensemble for a given realization of disorder (choice of $M$ marked states and their energies) with subsequent averaging over disorder realizations denoted by $[\ldots]_{\text {dis }}$. Note that the system size limit is taken prior to taking the time to infinity, which means that the time of a single spin flip diverges. The EdwardsAnderson order parameter in the frozen phase at $\beta>\beta_{f}$ is $q_{\mathrm{EA}}=1$. It is instructive to compare $q_{\mathrm{EA}}$ with the average overlap between bit string configurations $q_{\mathrm{RSB}}=\sum_{z, z^{\prime}} \exp \left\{-\beta\left[\mathcal{E}\left(z_{i}\right)+\mathcal{E}\left(z_{j}\right)\right]\right\} d_{z_{i} z_{j}}$, where $d_{z_{i} z_{j}}=$ $(1 / n) \sum_{k}\left(2 z_{i}^{k}-1\right)\left(2 z_{j}^{k}-1\right) . q_{\mathrm{RSB}} \neq 0$ indicates replica symmetry breaking in the model. Note that for the impurity band model $q_{\mathrm{RSB}}=0$ for any finite inverse temperature $\beta \sim \mathcal{O}\left(n^{0}\right)$.

It is instructive to compare the behavior of the impurity band model Eq. (3) with that of the random energy model (REM) [30], in which, instead of the relatively small number of $M \ll 2^{n}$ marked sates, all $2^{n}$ bit strings are randomly assigned energies drawn from a Gaussian distribution of width $\sqrt{n}$. The REM model demonstrates the freezing transition at $\beta_{f} \rightarrow 0$ and a replica symmetrybreaking transition at $\beta_{\mathrm{RSB}}=2 \sqrt{\ln 2}$ such that for $0<\beta<$ $\beta_{\mathrm{RSB}}$ we have $q_{\mathrm{EA}}=1$ and $q_{\mathrm{RSB}}=0$ and, for $\beta>\beta_{\mathrm{RSB}}$, $q_{\mathrm{EA}}=q=1$. In this sense, the impurity band model captures the nonergodic phase of the classical spin glass. Note that the finite value of the freezing temperature $\beta_{f}>0$ in the IB is the consequence of the bimodal distribution of classical energies which leaves a finite gap in the energy density between the marked states and the rest of the spectrum. This degeneracy of the classical spectrum is an important distinction of the IB model from the REM, and, 
therefore, the comparison between the quantum behavior of these two models has to be done with extreme care.

\section{B. Population transfer protocol}

Given a generic spin-glass model Eq. (2), we consider the following computational primitive: Given an initial bit string $z_{j}$ with an atypically low energy, we wish to produce other bit strings with energies in a narrow range $\Delta \mathcal{E}_{\mathrm{cl}}$ around the initial one. In general, this computation can be a difficult search problem if the number of bit strings of interest is exponentially small compared to $2^{n}$.

Inspired by the Hamiltonian-based approaches to quantum search [46] and optimization [2-4], we propose the following quantum PT)protocol: First preparing the system in a computational state $\left|z_{j}\right\rangle$ with classical energy $\mathcal{E}\left(z_{j}\right)$, we then evolve it under the Hamiltonian without fine-tuning the evolution time or the strength of the time-independent transverse field $B_{\perp}$. At the final moment, we projectively measure in the computational basis and check if the outcome $z$ is a "solution," i.e., $z \neq z_{j}$ and the energy $\mathcal{E}(z)$ is inside the window $\Delta \mathcal{E}_{\mathrm{cl}}$. The second term in the Hamiltonian (1) proportional to $B_{\perp}$ is responsible for the PT. It is usually referred to as a "driver Hamiltonian" in the quantum annealing literature [3].

We note that the output of PT $z$ can be used as an input of a classical optimization heuristic such as simulated annealing or parallel tempering in a "hybrid" optimization algorithm [47], where quantum and classical steps can be used sequentially to gain the complementary advantages of both [48].

In model (3), the most efficient classical algorithm is a purely random search with running time approximately $2^{n}$. We find that the typical run-time of the PT algorithm $t_{\mathrm{PT}}$ displays the following scaling dependence on $n$ :

$$
t_{\mathrm{PT}} \propto\left(\frac{2^{n}}{\Omega \log \Omega}\right)^{1 / 2} e^{n /\left(2 B_{\perp}^{2}\right)}
$$

Here, $\Omega \gg 1$ is the number of computational basis states within the target window of energies that contribute with comparable probabilities to the quantum state at the end of PT. The expression applies in the range of transverse fields $n^{1 / 2} \gg B_{\perp}-1=\mathcal{O}(1)$ [for arbitrary $B_{\perp}$, see Eq. (34)].

The dependence of $t_{\mathrm{PT}}$ on $\Omega$ is the same as in the multitarget Grover quantum algorithm that searches for $\Omega$ marked states starting from the fully symmetric state $|S\rangle=2^{-n / 2} \sum_{z}|z\rangle$. In the Hamiltonian version of this algorithm [46], one uses the projector to $|S\rangle$ as a driver: $H_{D}=w|S\rangle\langle S|$. This algorithm is proven to be optimal for problems without structure. We emphasize that according to Eq. (6) the exponential scaling of $t_{\mathrm{PT}}$ with $n$ differs from that in the Grover algorithm by a term $\sim B_{\perp}^{-2}$ that can be made arbitrary small at sufficiently large transverse fields.
The PT algorithm is qualitatively different from the quantum annealing, adiabatic optimization, and Hamiltonian implementation of a Grover search, because it exploits the structure of the excited energy spectrum. The PT Hamiltonian $H$ (1) is nonintegrable, and its eigenstates are delocalized in the low-energy manifold.

In the analytically tractable example considered here, the PT algorithm has new and potentially advantageous features compared to the Grover algorithm, whose Hamiltonian is integrable and all of its eigenstates but one are localized. Therefore, the quantum evolution resulting from the Grover Hamiltonian cannot form a massive superposition of $\Omega \gg 1$ solutions if it starts from a computational basis state. The algorithm must always start from the state $|S\rangle$. Moreover, Grover's algorithm performance is exponentially sensitive to fine-tuning of the weight of the driver $w$ on the scale $\delta w \sim 2^{-n / 2} \sqrt{\Omega}$. In contrast, the scaling of the run-time of PT (6) with $n$ is robust to the choice of $B_{\perp}$ that can take on a broad range of values for $B_{\perp} \gg 1$.

The nearly optimal (Grover-like) performance of the PT protocol is the consequence of the asymptotic orthogonality between the eigenstates in the marked state subspace to the rest of the Hilbert space, which suppresses the population transport from the marked states to the $\mathcal{O}\left(2^{n}\right)$ of states $|z\rangle$ with classical energies $E_{z}=0$ even at large $B_{\perp}$. Such an "orthogonality catastrophe" cannot be obtained within the perturbative in $B_{\perp}$ approach such as the FSA.

\section{Qualitative discussion of results}

Each marked state $\left|z_{j}\right\rangle$ is a deep local minimum of $\mathcal{E}(z)$ separated from other minima by a typical Hamming distance $n / 2$, while the separation from the nearest marked state is also extensive: $d_{\min }=\mathcal{O}(n)$ for $M=2^{\mu n}$ and $\mu<1$.

The transverse field $B_{\perp}$ gives rise to multiqubit tunneling between the states. The tunneling amplitudes from a given minimum to its neighbors located at a Hamming distance $d$ decrease exponentially with $d$, while the number of neighbors increases exponentially with $d$ for $d=\mathcal{O}(n)$. As a result, an eigenstate $\left|\psi_{\beta}\right\rangle$ of $H$ associated with the impurity band can become delocalized over a large subset of marked states $\mathcal{S}_{\beta}$ with size $1 \ll\left|\mathcal{S}_{\beta}\right| \propto M^{\alpha}$ and $0<\alpha \leq 1$. For $\alpha=0$, the eigenstate $\left|\psi_{\beta}\right\rangle$ is localized; for $\alpha=1$, the eigenstate is delocalized in the entire space of marked states. For $0<\alpha<1$, the eigenstate can be considered "nonergodic," and its support set $\mathcal{S}_{\beta}$ is sparse in the space of the marked states. We express the transition probability from $\left|z_{j}\right\rangle$ to $|z\rangle$

$$
P\left(t, z \mid z_{j}\right)=\left|\sum_{\beta}\left\langle z \mid \psi_{\beta}\right\rangle\left\langle\psi_{\beta} \mid z_{j}\right\rangle e^{-i E_{\beta} t}\right|^{2},
$$

in terms of the eigenstates and corresponding eigenvalues of $H$, where $H\left|\psi_{\beta}\right\rangle=E_{\beta}\left|\psi_{\beta}\right\rangle$. In the delocalized phase, 
for a given state $\left|z_{j}\right\rangle$ there exists a large set of eigenstates $\left|\psi_{\beta}\right\rangle$ that have peaks at $\left|z_{j}\right\rangle$. These eigenstates possess important properties $[21,22,49]$ : They have largely overlapping supports $\cap_{\beta} \mathcal{S}_{\beta} \approx \mathcal{S}\left(z_{j}\right)$, and they are close in energy, thus forming a narrow miniband. The miniband width $\Gamma$ may be interpreted as the inverse scrambling time and determines the width of the plateau in the Fourier transform of the typical transition probability $\tilde{P}\left(\omega, z \mid z_{j}\right)$ [21,50]. In other words, the significant PT of $P\left(t, z \mid z_{j}\right)$ from the initial marked state $\left|z_{j}\right\rangle \in \mathcal{S}$ into the other states of the same miniband $\mathcal{S}$ occurs over the time $t_{\mathrm{PT}} \sim 1 / \Gamma$. The window $\Delta \mathcal{E}_{\mathrm{cl}}$ is related to the miniband width $\Gamma$.

Understanding the properties of nonergodic delocalized states is crucial for describing the dynamics of quantum spin glasses driven by many-body coherent tunneling processes. Developing its microscopic theory is a challenging problem. This paper studies the transport problem in an IB model (3) by making use of the down-folded Hamiltonian in the marked state subspace derived in Secs. III and V. While the original Hamiltonian (1) is sparse in the basis of states $|z\rangle$ (it couples only states separated by Hamming distance 1), the down-folded Hamiltonian $\mathcal{H}(41)$ is a dense $M \times M$ matrix.

The transverse field leads to a uniform shift $\sim B_{\perp}^{2}$ of the marked state energies as shown in Sec. V, Eqs. (36) and (37). Diagonal elements of $\mathcal{H}_{i i}$ are given by the marked state energies counted off from the center of the shifted impurity band. Their probability density function (PDF) is assumed to be exponentially bounded with some width $W$.

Each pair of marked states is coupled via multiqubit tunneling. The off-diagonal matrix elements $\mathcal{H}_{i j}=$ $V\left(d_{i j}\right) \cos \phi\left(d_{i j}\right)$ are completely determined by the Hamming distance $d_{i j}$ between the marked states $z_{i}$ and $z_{j}$. The amplitude $V(d)$ decays steeply with $d$, inversely proportional to a square root of $\left(\begin{array}{l}n \\ d\end{array}\right)$ [see Eq. (42)]. The phase $\phi$ shown in Fig. 5 monotonically increases by $\mathcal{O}(1)$ when $d$ is changed by 1 . In the analysis of spectral properties of $\mathcal{H}_{i j}$, the quantity $\cos \phi\left(d_{i j}\right)$ can be replaced by a random sign. The explicit form of $V(d)$ and $\phi(d)$ is obtained using the WKB theory of collective spin tunneling. At $B_{\perp}>1$, the tunneling paths correspond to long spin-flip sequences connecting the initial and final states. They include many loops passing through the states with $\mathcal{E}(z)=0$ that are neglected in the FSA.

The typical matrix element between the two marked states is $V_{\text {typ }} \sim n^{2} 2^{-n / 2} e^{-n /\left(4 B_{\perp}^{2}\right)}$. The typical matrix element between a given marked state and its nearest neighbor is also exponentially small in $n$, but it is exponentially larger than the value $V_{\text {typ }}$. This fact corresponds to a strong hierarchy of the off-diagonal matrix elements of $\mathcal{H}_{i j}$ which is a signature of their heavy-tailed probability density function $[33,37]$. Such matrices are called Levi matrices.
The PDF of the rescaled squared amplitudes $w_{i j}=$ $V^{2}\left(d_{i j}\right) / V_{\text {typ }}^{2}$ derived in Sec. VI B is

$$
\operatorname{PDF}(w)=\frac{1}{w^{2} \sqrt{\pi \log w}}, \quad w \in[1, \infty) .
$$

The particular form of scaling is the direct consequence of the fact that our problem has no "structure": The tunneling matrix elements depend only on the Hamming distance, and marked states are chosen at random.

The key difference of the ensemble of matrices $\mathcal{H}_{i j}$ from Levy matrices studied in the literature [33-35,37] is that the dispersion $W$ of the diagonal matrix elements is much larger than the typical magnitude of the off-diagonal elements $V_{\text {typ }}$. Therefore, $\mathcal{H}_{i j}$ can be called preferred basis Levi matrices (PBLMs).

We note that the existence of heavy tails in the PDF of the off-diagonal matrix elements of the down-folded Hamiltonian $\mathcal{H}$ is due to the infinite dimension of the Hilbert space of the original problem (1) for $n \rightarrow \infty$. This result happens because the exponential decay of the matrix elements with the Hamming distance $d$ is compensated by the exponential growth of the number of states at the distance $d$ from a given state. We believe that the PBLM structure is a generic feature of the effective Hamiltonians for PT at the tail of the density of states in quantum spinglass problems.

Unlike the standard Levi ensemble, the eigenstates of PBLMs allow for the existence of nonergodic delocalized states when the width $W$ is much bigger than the largest offdiagonal matrix element in a typical row of $\mathcal{H}_{i j}$ and much smaller than the largest off-diagonal element in a matrix

$$
V_{\text {typ }} M^{1 / 2} \ll W \ll V_{\text {typ }} M .
$$

For smaller dispersion $W \lesssim V_{\text {typ }} M^{1 / 2}$, the matrix eigenstates are ergodic, while for $W \gtrsim V_{\mathrm{typ}} M$ the eigenstates are localized. Such a phase diagram resembles the one in the Rosenzweig-Porter (RP) model $[21,36]$. The difference of RP from PBLM is that the statistics of the off-diagonal matrix elements in the RP ensemble are Gaussian [51] rather than polynomial (8). In this paper, we focus on exploring PT transfer within the nonergodic delocalized phase, which is more likely to generalize to other models. We note that the localized phase does not support population transfer.

Because of the PBLM structure of the Hamiltonian $\mathcal{H}$, one can expect that the run-time of the PT protocol $t_{\mathrm{PT}}$ will have a heavy-tailed PDF whose form is of practical interest. It is closely related to the PDF of the miniband widths $\Gamma \sim 1 / t_{\mathrm{PT}}$. We obtain the $\operatorname{PDF}(\Gamma)$ by making use of the cavity method for random symmetric matrices [32,33,35,52].

In previous work, the cavity equations are solved only in their linearized form, i.e., near the localization transition. We are able to solve fully nonlinear cavity equations in the 
delocalized nonergodic phase. We obtain the boundaries of the phase in terms of the ratio of $W / V_{\text {typ }}$ and also the form of $\mathcal{P}(\Gamma)$ inside the phase. It is given by the alpha-stable Levi distribution $[33,53]$ with the tail index 1 , most probable value $\Gamma_{\text {typ }}=V_{\text {typ }}(\pi \Omega \log \Omega / 4)^{1 / 2}$, and characteristic dispersion $\pi \Gamma_{\text {typ }} /(4 \log \Omega)$, where $\Omega$ is the typical number of states in the miniband. This number $\Omega=$ $\left(\pi M V_{\text {typ }} / W\right)^{2}$ is a square function of the ratio of the typical tunneling matrix element $V_{\text {typ }}$ to the level separation $W / M$. In a nonergodic delocalized phase, $M \gg \Omega \gg 1$, and the typical PT time $t_{\mathrm{PT}} \sim 1 / \Gamma_{\text {typ }}$ obeys the condition

$$
(M \log M)^{-1 / 2} \ll t_{\mathrm{PT}} V_{\mathrm{typ}} \sim(\Omega \log \Omega)^{-1 / 2} \ll 1 .
$$

We build on the observations made in the IB model and provide qualitative arguments that PT will have a quadratic speedup over QMC in some quantum search problems where tunneling is a computational bottleneck.

It is instructive to connect the phase boundaries of the NEE regime to that of the Rosenzweig-Porter random matrix model. We proceed by parametrizing the scaling of the width $W$ with $M$ as follows:

$$
W=\lambda M^{\gamma / 2} V_{\mathrm{typ}}
$$

where $\gamma$ is a real non-negative parameter, $V_{\text {typ }}$ is the typical off-diagonal matrix element, and $\lambda$ is an auxiliary constant of the order of one. A direct analogy between the scaling exponent $\gamma$ and its counterpart in the RP model can now be made. It determines the number of matrix elements resonant with the given one. $\gamma>2$ correspond to a vanishing number of resonances and, therefore, a localized phase. The nonergodic phase in these notations corresponds to $1<\gamma<2$, where the number of resonances scales with the system size yet remains measure zero compared to the total number of levels in the system. $\gamma \leq 1$ corresponds to the ergodic phase.

\section{DOWN-FOLDING INTO THE SUBSPACE OF THE MARKED STATES AND NONLINEAR EIGENPROBLEM}

The driver Hamiltonian $H_{D}$ in Eq (1) connects bit strings that are separated by a Hamming distance $d=1$. We note that, on one hand, marked states are separated by large Hamming distances $d_{i j}$ with typical value $d=n / 2$. Therefore, a pair of marked states $|i\rangle$ and $|j\rangle$ is coupled by elementary spin-flip processes corresponding to high orders $\left(H_{D}\right)^{k}$ of the driver Hamiltonian with $k \geq d_{i j}$. On the other hand, the resolvent of the driver Hamiltonian

$$
G(E)=\frac{1}{E-H_{D}}
$$

connects directly every pair of marked states. Furthermore, because $H_{D}$ is invariant under permutations of bits, the matrix elements $G_{i j}(E)=\left\langle z_{i}|G(E)| z_{j}\right\rangle$ depend only on the Hamming distance $d_{i j}$ between the corresponding states. They are exponentially small in $n$ for extensive $d_{i j}=\mathcal{O}(n)$. Therefore, one might expect that under certain conditions the quantum evolution stays approximately confined to the marked state subspace and can be naturally described by the down-folded Hamiltonian whose $M \times M$ matrix representation is dense in the basis of marked states.

We use the identity

$$
G(E) H_{\mathrm{cl}}|\psi\rangle=|\psi\rangle,
$$

where $E$ and $|\psi\rangle$ are an eigenvalue and the corresponding eigenvector of $H$, respectively. We introduce a new vector

$$
|\mathcal{A}\rangle=\sqrt{H_{\mathrm{cl}}}|\psi\rangle
$$

that has no support in the subspace orthogonal to that of marked states. Then, multiplying both parts of Eq. (13) by $\sqrt{H_{\mathrm{cl}}}$, we obtain after simple transformations

$$
\left(H_{\mathrm{cl}}+\Lambda\right)|\mathcal{A}\rangle=E|\mathcal{A}\rangle,
$$

where

$$
\Lambda=\sqrt{H_{\mathrm{cl}}} H_{D} G(E) \sqrt{H_{\mathrm{cl}}} .
$$

The operator $\Lambda$ plays the role of a "driver Hamiltonian" in the down-folded picture, and it couples states in the marked subspace.

Equation (15) can be written in matrix form (see the Appendix A for details)

$$
\sum_{j=1}^{M} \mathcal{H}_{i j}(E) \mathcal{A}_{j}=E \mathcal{A}_{i}
$$

where $\mathcal{A}_{i}=\left\langle\mathcal{A} \mid z_{i}\right\rangle$ and $\mathcal{H}_{i j}$ is a dense symmetric $M \times M$ matrix

$$
\mathcal{H}_{i j}(E)=\delta_{i j} \mathcal{E}\left(z_{i}\right)+\sqrt{\mathcal{E}\left(z_{i}\right) \mathcal{E}\left(z_{j}\right)} c_{i j}(E) .
$$

Here, $\delta_{i j}$ is the Kronecker delta, and

$$
c_{i j}(E)=c\left(E,\left|z_{i}-z_{j}\right|\right)=\left\langle z_{i}\left|H_{D} \frac{1}{H_{D}-E}\right| z_{j}\right\rangle
$$

is a coupling coefficient that depends only on a Hamming distance $\left|z_{i}-z_{j}\right|$ between the bit strings $z_{i}$ and $z_{j}$.

We note that Eq. (17) has the form of a nonlinear eigenproblem. A solution of Eq. (17) for the $M$-dimensional vector $|A\rangle$ with a nonzero norm requires 


$$
\operatorname{det}[\mathcal{H}(E)-I E]=0,
$$

where $I$ is the identity matrix. Because the down-folded Hamiltonian $\mathcal{H}(E)$ explicitly depends on the energy $E$, different roots $E_{\beta}$ of Eq. (20) correspond to different Hamiltonian matrices $\mathcal{H}_{i j}\left(E_{\beta}\right)$. This correspondence can be understood from the fact that the original $2^{n} \times 2^{n}$ Hamiltonian (1) couples the $M$-dimensional marked state subspace to the rest of the Hilbert space. Therefore, the projections of the eigenvectors $\left|\psi_{\beta}\right\rangle$ of $H$ onto the subspace are, in general, neither normalized nor orthogonal. The same is true for the corresponding vectors $\left|\mathcal{A}_{\beta}\right\rangle=\sqrt{H_{\mathrm{cl}}}\left|\psi_{\beta}\right\rangle$. The normalization condition for the projections has the form (see Appendix A for details)

$$
\sum_{j, i=1}^{M} \frac{1}{Q_{j i}\left(E_{\beta}\right)} \psi_{\beta}\left(z_{j}\right) \psi_{\beta}\left(z_{i}\right)=1,
$$

where

$$
\frac{1}{Q_{i j}(E)}=\sqrt{\mathcal{E}\left(z_{i}\right) \mathcal{E}\left(z_{j}\right)} \frac{d}{d E}\left(\frac{\mathcal{H}_{i j}(E)}{E}\right) .
$$

This condition along with Eqs. (17)-(20) completely defines the eigenvector projections onto the marked state subspace and the corresponding eigenvalues.

We observe that there are exactly $M$ roots $E_{\beta}$ of Eq. (20) that originate from $M$ classical energies of the marked states $\mathcal{E}\left(z_{j}\right)$ at $B_{\perp}=0$. These eigenvalues and the corresponding eigenstates are the sole focus of our study. Here, we just mention briefly that the rest of the states originate in the limit $H_{\mathrm{cl}} \rightarrow 0$ from the eigenstates of the driver Hamiltonian whose energy levels $-B_{\perp}(n-2 m)$ (shown in Fig. 1) correspond to the total spin- $x$ projections $n-2 m \in[-n, n]$. The levels $-B_{\perp}(n-2 m)$ have degeneracy $\left(\begin{array}{l}n \\ m\end{array}\right)$, which is partially lifted due to the coupling to the impurity band with $M$ states. The splitting of the driver energy levels $-B_{\perp}(n-2 m)$ increases as a function of the transverse field in the vicinity of "resonances" with the levels of the impurity band where $B_{\perp}(n-2 m) \approx-n$ for integer values of $m$. At resonance, the eigenstates of the driver with total spin- $x$ projection $n-2 m$ are strongly hybridized with the marked states $\left|z_{j}\right\rangle$. As discussed below, the width of the resonances remains exponentially small in $n$ for $B_{\perp}=\mathcal{O}\left(n^{0}\right)$. In Fig. 6, we plot the evolution of the energy spectrum of the Hamiltonian $H$ as a function of the transverse field for the case of two impurity states $M=2$.

\section{COUPLING COEFFICIENTS IN THE DOWN-FOLDED HAMILTONIAN}

The coupling coefficient $c_{i j}(E) \equiv c\left(E, d_{i j}\right)$ for $i \neq j$ determines the off-diagonal matrix element of the downfolded Hamiltonian (18) corresponding to the tunneling transition that connects marked states $\left|z_{i}\right\rangle$ and $\left|z_{j}\right\rangle$. In the IB model, the tunneling matrix element depends only on the Hamming distance $d_{i j}$ between the states. It can be calculated in the explicit form from Eq. (19). For this calculation, we use the basis of eigenstates $|x\rangle$ of the driver Hamiltonian $H_{D}|x\rangle=H_{D}^{x}|x\rangle$ in Eq. (19). They correspond to bit strings $x=\left(x^{1}, \ldots, x^{n}\right)$ of individual qubits polarized in the positive $x^{a}=0$ and negative $x^{a}=1$ direction of the $x$ axis. The eigenvalues of the driver $H_{D}^{x}=-B_{\perp}\left(n-2 h_{x}\right)$ depend only on the Hamming weight of the bit strings $x$. Therefore, one can perform explicitly the partial summation over basis vectors $|x\rangle$ in Eq. (19) under the conditions that $\sum_{a} x^{a}=k$ for all bit positions $a$ such that $z_{j}^{a} \neq z_{i}^{a}$ and $\sum_{a} x^{a}=l$ for all $a$ where $z_{j}^{a}=z_{i}^{a}$. Finally, the result (19) can be written as a double sum over $k \in\left(0, n-d_{i j}\right)$ and $l \in\left(0, d_{i j}\right)$ :

$c_{i j}(E)=\sum_{k=0}^{n-d_{i j}} \sum_{l=0}^{d_{i j}}\left(\begin{array}{l}n \\ k\end{array}\right)\left(\begin{array}{c}n-d_{i j} \\ l\end{array}\right) \frac{(-1)^{l} 2^{-n}}{1+\frac{E}{B_{\perp}(n-2 k-2 l)}}$.

Here, $d_{i j}$ is the Hamming distance between bit strings $z_{i}$ and $z_{j}$. Plots of coupling coefficients as a function of Hamming distance $d$ based on Eq. (23) are given in Fig. 2. They display qualitatively different behavior depending on the value of the parameter $n B_{\perp} /|E|$.

For $n B_{\perp} /|E|<1$, the coefficient $c(E, d)$ decays exponentially with $d$ in the entire range of values $d \in[0, n]$. For $n B_{\perp} /|E|>1$, the coefficient decays until $d \sim n / 2$, corresponding to a minimum overlap between the marked states, and then begins to grow. For large transverse field $B_{\perp} \gg 1$, the behavior with $d$ is nearly symmetric with respect to $d=n / 2$, and to leading order it does not depend on $B_{\perp}$. Unfortunately, the expression (23) is quite involved and is not suitable for the study of the asymptotic properties of the population transfer in the limit of large $n$.

For a very weak transverse field $B_{\perp} \ll n^{-1 / 2}$, using the perturbation theory in $B_{\perp}$ to the leading order, one can obtain a standard expression [18] for the coupling coefficient: $|c(E, d)| \simeq d !\left(B_{\perp} / n\right)^{d}$. It is given by the sum of the transition amplitudes over the $d$ ! shortest paths between the states $\left|z_{i}\right\rangle$ and $\left|z_{j}\right\rangle$ separated by a Hamming distance $d$. Intermediate states $|z\rangle$ along each path correspond to $\mathcal{E}(z)=0$, while energies of initial and final states are $-n$ (see Fig. 1).

For larger transverse-field values (but still $B_{\perp} \ll 1$ ), the perturbative expression in the small- $B_{\perp}$ limit can be modified to include the range of $B_{\perp}=\mathcal{O}\left(n^{0}\right)$ but $B_{\perp} \ll 1$. In that range,

$|c(E, d)| \simeq d !\left(\frac{B_{\perp}}{n}\right)^{d} e^{B_{\perp}^{2}\left\{\left[\left(d^{2}(3 n-2 d)\right) /\left(6 n^{2}\right)\right]+[n /(12)]\right\}}$.

One can see that for small $B_{\perp}$ the matrix element falls down with $d$ extremely steeply despite the presence of the 


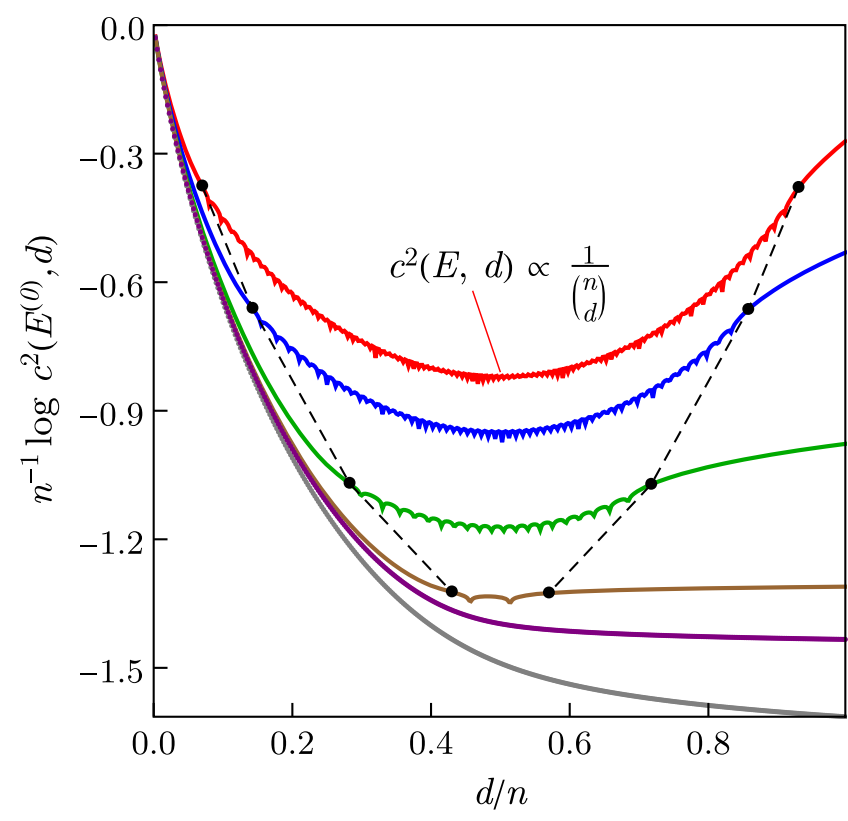

FIG. 2. Colored lines show the dependence of the rescaled logarithm of the coupling coefficient $n^{-1} \log c^{2}(E, d)$ [Eq. (23)] on the rescaled Hamming distance $d / n$ for $n=400$. The energy $E$ is set to the value $E^{(0)} \simeq-n-B_{\perp}^{2}$ that reflects the overall shift of the impurity band due to the transverse field [cf. Eqs. (36) and (37)]. Different colors correspond to different values of the transverse field $B_{\perp}=1.93$ (red), 1.43 (blue), 1.11 (green), 1.01 (brown), 0.99 (purple), and 0.95 (gray). The scale along the $y$ axis suggests that $c\left(E^{(0)}, d\right)$ scales exponentially with $n$ for $d / n=\mathcal{O}\left(n^{0}\right)$. The inset shows the leading-order factor in the $d$ dependence of the coupling coefficient for $B_{\perp}>|E| / n$ [cf. Eq. (33)]. Black dots show the boundaries $d=n / 2-m_{0}$, $n / 2+m_{0}$ of the region of the oscillatory behavior of $c(E, d)$ with $d$ given by the WKB theory [Eq. (32)] (see Appendix B for details).

factorial factor $d$ ! in Eq. (24). We note that this perturbation (FSA) expression is qualitatively valid in the range $B_{\perp}<|E / n| \ll 1$. It gives a correct leading-order form of the mobility edge in quantum REM [18,27-29] at small $B_{\perp} \ll 1$.

For transverse field $B_{\perp}>|E| / n$, the dependence of $c_{i j}(E)$ on $d_{i j}$ changes qualitatively. It becomes nonmonotonic, reaching its minimum at the point $n / 2$ of minimum overlap between the bit strings $z_{i}$ and $z_{j}$. In a certain region around the minimum, it has oscillatory behavior, as seen in Fig. 2. The boundary of this region is shown with black dots. The details of the behavior in the oscillatory region are shown in Fig. 4. The exponential dependence of the envelope of $c(E, d)$ on $d$ is captured by the factor $1 /\left(\begin{array}{l}n \\ d\end{array}\right)$ and is independent of the transverse-field strength. This region of $d$ and values of $B_{\perp}>|E| / n$ are of the most relevance to the transport in nonergodic minibands which is of central interest in this paper.

\section{A. WKB calculation of coupling coefficients}

In this paper, we develop an approach (described in Appendix B) based on the WKB theory for large spin [54] to calculate the coefficient $c(E, d)$ for $n \gg 1$ and arbitrary values of transverse fields $B_{\perp}$ without relying on the perturbation theory in $B_{\perp}$. The coefficient $c_{i j}(E)$ can be expressed in terms of the operator of the total spin- $x$ projection $S_{x}=1 / 2 \sum_{j=1}^{n} \sigma_{x}^{j}$ :

$$
c_{i j}(E)=\delta_{i j}-E\left\langle z_{i}\left|\left(E+2 B_{\perp} S_{x}\right)^{-1}\right| z_{j}\right\rangle .
$$

We utilize the basis of eigenstates $|m\rangle$ of the operator $S_{z}=\sum_{k=1}^{n} \sigma_{z}^{k}$ corresponding to its eigenvalues $m \in$ $[-n / 2, n / 2]$ and the maximum value of the total spin $S=n / 2$ :

$$
S_{z}|m\rangle=m|m\rangle, \quad m=-n / 2, \ldots, n / 2 .
$$

The state $|n / 2-d\rangle$ is a normalized sum of all computational basis states $|z\rangle$ with $d$ spins pointing in the negative $z$ direction and $n-d$ spins pointing in the positive $z$ direction $(m=n / 2-d)$ :

$$
|n / 2-d\rangle=\frac{1}{\sqrt{\left(\begin{array}{c}
n \\
d
\end{array}\right)}} \sum_{z \in\{0,1\}^{n}} \delta_{|z|, d}|z\rangle .
$$

Here, $|z|=\sum_{k=1}^{n} z^{k}$ and $\delta_{k, d}$ is a Kronecker delta.

Because the coefficients $c_{i j}(E)$ in Eq. (23) depend only on the Hamming distance $\left|z_{i}-z_{j}\right|$ between the bit strings $z_{i}$ and $z_{j}$, we can assume, without the loss of generality, that in Eq. (25) one of the bit strings, e.g., $\left|z_{j}\right\rangle$, corresponds to all individual spins pointing in the positive $z$ direction:

$$
\left|z_{j}\right\rangle=|00 \ldots 0\rangle \equiv|n / 2\rangle \quad\left(m_{z}=n / 2\right) .
$$

The main observation is that we can pick, instead of the state $\left|z_{i}\right\rangle$, any computational basis state $|z\rangle$ whose Hamming weight satisfies the condition $|z|=\left|z_{i}\right|$ without changing the value of the coefficient $c_{i j}(E)=c\left(E,\left|z_{i}\right|\right)$. Therefore, averaging both sides of Eq. (25) over the states $\left|z_{i}\right\rangle$ that satisfy the condition $\left|z_{i}\right|=d$ for some integer $d \in[0, n]$, we obtain

$$
c(E, d)=\delta_{d, 0}-\frac{E}{\sqrt{\left(\begin{array}{l}
n \\
d
\end{array}\right)}} G_{(n / 2)-d,(n / 2)}(E)
$$

Here, $\quad G_{m,(n / 2)}(E)=\left\langle m\left|\left(E+2 B_{\perp} S_{x}\right)^{-1}\right| n / 2\right\rangle \quad$ are the matrix elements of the resolvent (12) of the transversefield Hamiltonian $H_{D}$ between the states (27) that belong to a maximum total spin subspace $S=n / 2$.

As shown below, for typical instances of the ensemble of Hamiltonians $H$, the Hamming distance from a randomly selected marked state to its closest neighbor is an extensive 
quantity $\mathcal{O}(n)$. Therefore, the above off-diagonal matrix elements of the resolvent can be analyzed in a semiclassical approximation corresponding to $S=n / 2 \gg 1$. This approximation for the quantum propagator of a large spin and diagonal elements of the resolvent is considered in Refs. [55,56] using the spin coherent state path-integral representation. The analysis in these papers is quite involved, because the path-integral formulation requires a careful treatment of the fluctuation determinant and a socalled Solari-Kochetov correction in the action. Also, these results are focused on a general case of a large spin Hamiltonian and consider only diagonal elements of the resolvent. Because of this difference, instead of trying to extend the results in Refs. [55,56] to our case, we follow a different path.

The resolvent satisfies the equation

$$
I-2 B_{\perp} S_{x} G(E)=E G(E),
$$

where $I$ is the identity operator. We write this equation in the basis of states $|m\rangle$ [Eq. (26)]. From Eq. (12), we obtain

$$
\begin{array}{r}
\delta_{m,(n / 2)}+\sum_{s= \pm 1} u(m-s / 2) G_{m+s,(n / 2)}=E G_{m,(n / 2)}, \\
u(m)=-B_{\perp} \sqrt{L^{2}-m^{2}}, \quad L=\frac{n+1}{2} .
\end{array}
$$

In the limit of large $n \gg 1$, we solve this equation using the discrete WKB approximation method [54,57]. In the WKB analysis of Eq. (30), the function $2 u(m)$ plays the role of an effective potential for the classical system with coordinate $m$ and energy $E$. For $2 u(m)>E$ the WKB solution for the resolvent $G_{m, n / 2}(E)$ displays an oscillatory behavior with $m$, while for $2 u(m)<E$ it exponentially increases with $m$. The boundaries of the oscillatory region $m \in\left[-m_{0}(E), m_{0}(E)\right]$ are "turning points" of the classical motion and are given by the condition $2 u\left(m_{0}\right)=E$ (see Fig. 3) where

$$
m_{0}=\sqrt{L^{2}-\left(\frac{E}{4 B_{\perp}}\right)^{2}} .
$$

In Fig. 4, we plot the comparison between the coefficient $c(E, d)$ computed based on the exact expression (23) and the WKB asymptotic (details of the WKB analysis are given in Appendix B).

In what follows, we are interested in the region $d \in$ $\left[n / 2-m_{0}, n / 2+m_{0}\right] \quad$ with $\quad m_{0} \simeq \sqrt{(n / 2)^{2}-\left(E / B_{\perp}\right)^{2}}$ defined by the condition $u\left(m_{0}\right)=E$. This region is of oscillatory behavior of $c(E, d)$ with $d$, where the leadingorder exponential dependence on $n$ is given by the expression

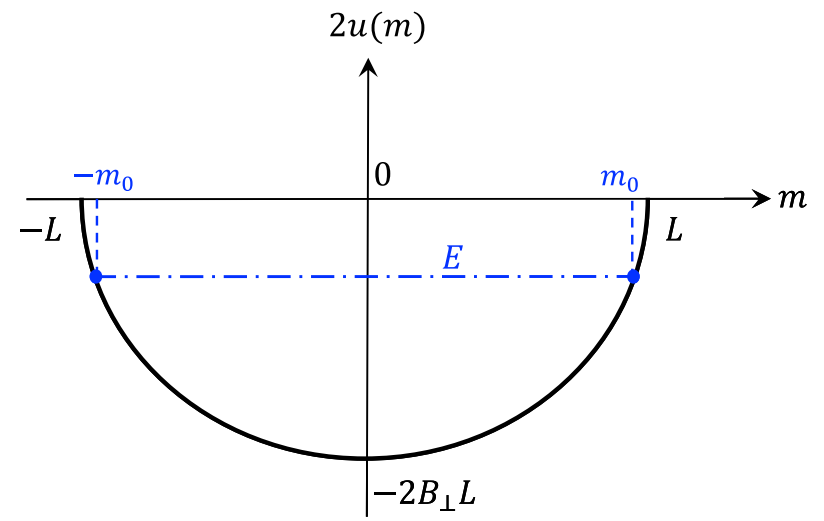

FIG. 3. The black line shows the plot of $2 u(m)(31)$ vs $m$ between the interval boundaries $\pm m=L=(n+1) / 2$. The horizontal dashed-dotted blue line depicts the region of oscillatory behavior of $G_{m, n / 2}(E)$ with $m$ for a given $E$ described by the WKB solution (33) [see also Eq. (B5) in Appendix B] and shown in Fig. 4. The boundaries of this region are the turning points $m= \pm m_{0}(E)$ given by Eq. (32) and depicted with blue dots. The regions of $m \in\left[m_{0}(E), L\right] \cup\left[-L,-m_{0}(E)\right]$ correspond to the exponential growth of $G_{m, n / 2}(E)$ with $m$ (or decrease with $d=n / 2-m$ ). The WKB solution for the right region is given in Eq. (B10).

$$
c(E, d) \propto \frac{1}{\sqrt{\left(\begin{array}{l}
n \\
d
\end{array}\right)}} e^{-n \theta\left(B_{\perp}\right)} \sin \phi(E, d),
$$

with the prefactor given in Appendix B, Eqs. (B23) and (B24).

The function $\theta\left(B_{\perp}\right)$ in Eq. (33) equals

$$
\theta\left(B_{\perp}\right)=\frac{2 \operatorname{arctanh}\left(B_{\perp}^{-1}\right)+B_{\perp} \ln \left(1-B_{\perp}^{-2}\right)}{4 B_{\perp}} .
$$

It behaves at a large argument as $\theta \simeq 1 /\left(4 B_{\perp}^{2}\right)$.

An explicit form of the WKB phase $\phi(E, d)$ in Eq. (33) is given in Appendix B, Eq. (B11). The dependence of the phase on $d$ for different values of $B_{\perp}$ is shown in Fig. 5 . This phase varies by $\mathcal{O}(1)$ when $d$ is changed by 1 , and it is responsible for fast oscillation of the coupling coefficient with the Hamming distance between marked states $d$. Its dependence on $d$ simplifies in the limit of large transverse field $B_{\perp} \gg 1$ :

$$
\phi(E, d) \simeq \frac{\pi d}{2}-\frac{\pi n}{4} \frac{\chi(1 / 2-d / n)}{B_{\perp}}
$$

where $\chi(x) \simeq 1-2 \arcsin (x) / \pi+\mathcal{O}\left(n^{-1}\right)$. The second term in Eq. (35) is much smaller than the first one and varies very little when $d$ is changed by 1 . A predominately linear dependence of $\phi(E, d)$ on $d$ at large fields can be seen in Fig. 5. This property is important in the analytical study of population transfer. 


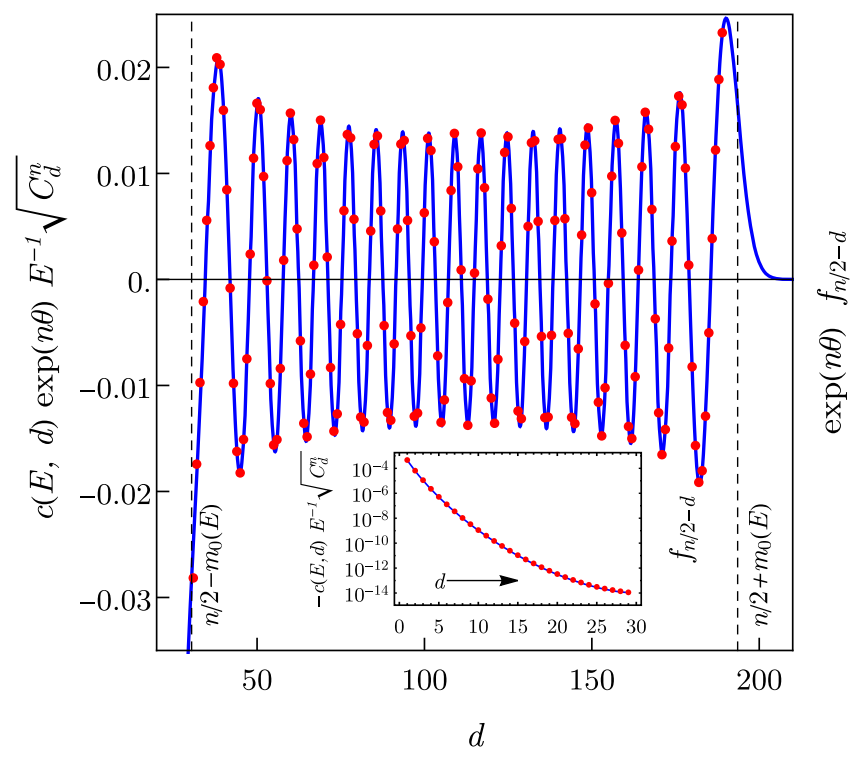

FIG. 4. The blue curve shows the $d$ dependence of the (rescaled) coupling coefficients $c(E, d)$ computed from the exact expression (23) with $n=224$ and $E=-226.15$. We denote the binomial coefficient as $\left(\begin{array}{l}n \\ d\end{array}\right) \equiv C_{d}^{n}$. The transverse field is $B_{\perp}=1.459$. For this value of $B_{\perp}$, the impurity band levels $\mathcal{E}\left(z_{j}\right)$ lie approximately in the middle of the interval between the $p=34$ th and $p=35$ th excited energy levels $-B_{\perp}(n-2 p)$ of the driver Hamiltonian. Red points depict the $d$ dependence of the same rescaled coefficients $c(E, d)$ given by $G_{n / 2-d, n / 2} \exp (n \theta)$ and determined by the asymptotic WKB expressions given in Appendix B [see Eqs. (B10) and (B13)]. Dashed lines indicate the boundaries of the oscillatory behavior of the WKB solution [Eq. (B9)]. The inset shows the plot for the exponential $d$ dependence of the rescaled coupling coefficient $-c(E, d)$ in the region of its monotonic behavior $d \in$ $\left[1, n / 2-m_{0}(E)\right]$ [cf. Eqs. (B13) and (29)]. The solid blue line corresponds to the exact expression (23), while the approximate WKB solution is shown with red points.

For large transverse fields, the magnitude of the squared coupling coefficient (33) can be estimated to exponential accuracy as $c^{2}(E, d) \sim \exp \left[-n /\left(2 B_{\perp}^{2}\right)\right] /\left(\begin{array}{l}n \\ d\end{array}\right)$. We note that the number of marked states $M_{d}$ accessible via all possible $d$-bit flips from a given state is $M_{d}=M 2^{-n}\left(\begin{array}{c}n \\ d\end{array}\right)$. Therefore, the leading-order dependence of the coupling coefficient on $d$ is proportional to $1 / \sqrt{M_{d}}$. As shown later, in the limit of large transverse fields, this dependence leads to a nearly Grover complexity of the PT algorithm, up to a factor $\sim \exp \left[-n /\left(4 B_{\perp}^{2}\right)\right]$, which gives a very small correction to Grover scaling for large $B_{\perp}$. However, when $d$ decreases below the boundary value $d<n / 2-m_{0}$, the coupling coefficient grows exponentially faster than $1 / \sqrt{M_{d}}$, as follows from the discussion in Appendix B [cf. Eq. (B13)].

\section{DOWN-FOLDED HAMILTONIAN NEAR THE CENTER OF THE IMPURITY BAND}

The coupling coefficients $c(E, d)$ [Eq. (23)] decay exponentially with Hamming distances for $d=\mathcal{O}(n)$

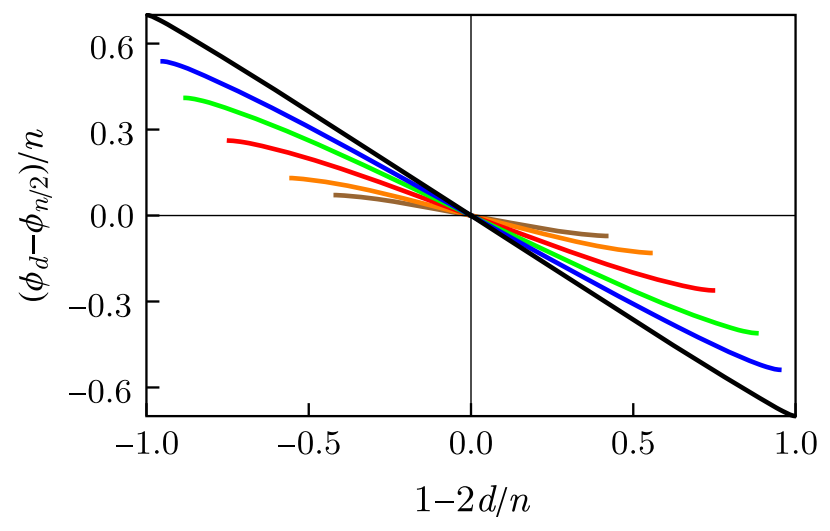

FIG. 5. Plots of the WKB phase $\phi_{d} \equiv \phi(E, d)$ of the oscillations of the coupling coefficient $c(E, d)$ with the Hamming distance $d$ for a number of qubits $n=1000$. Both axes are rescaled by $n$. The phase is plotted relative to its value at $d=n / 2$. We set the energy $E=E^{(0)}$, where $E^{(0)} \simeq-n-B_{\perp}^{2}$ reflects the overall shift of the impurity band due to the transverse field [cf. Eqs. (36) and (37)]. Different color curves correspond to different values of $B_{\perp}>$ $|E| / n$ with $B_{\perp}=1.1$ (brown), $B_{\perp}=1.2$ (orange), $B_{\perp}=1.5$ (red), $B_{\perp}=2.1$ (green), $B_{\perp}=3.2$ (blue), and $B_{\perp}=10$ (black). Each curve varies in its own range $n / 2-d \in\left[-m_{0}, m_{0}\right]$, where $m_{0}$ is given in Eq. (32) and determines the region of oscillatory behavior of the coupling coefficients (see Appendix B for details). For $B_{\perp} \simeq 1$, the region of oscillatory behavior shrinks to a point $d \simeq n / 2$. In the limit of large values of $B_{\perp} \gg 1$, this behavior occupies almost the entire range $d \in[0, n]$.

(see details in Sec. IV). Marked states are selected at random, and Hamming distances between them are of the order of $n$ when the number of the states $M$ is exponentially smaller than $2^{n}$. Because the off-diagonal matrix elements of the down-folded Hamiltonian $\mathcal{H}_{i j}(E) \propto c\left(E, d_{i j}\right)$, they are exponentially small in $n$. At the same time, the width of the distribution of energies of the marked states $\mathcal{E}\left(z_{j}\right)=$ $-n+\epsilon_{j}$ is also assumed to be very small: $W \ll B_{\perp}$ (it is exponentially small in $n$ for the cases of interest). Therefore, we can solve the nonlinear eigenvalue problem [Eqs. (17)-(20)] by an iterative approach treating the offdiagonal part of $\mathcal{H}(E)$ and terms $\propto \epsilon_{j}$ as a perturbation. Details are given in Appendix C.

At zeroth order in the perturbation, the down-folded Hamiltonian $\mathcal{H}_{i j}^{(0)}(E)=\delta_{i j} n[c(E, 0)-1]$ has one $M$-fold degenerate energy level $E^{(0)}$ that is a root of the equation $\mathcal{H}_{i j}^{(0)}(E)=E$ that originates from the marked state energy $E^{(0)} \rightarrow-n$, in the limit of $B_{\perp} \rightarrow 0$. Using $c(E, 0)$ from Eqs. (19) and (23), the explicit form of the equation for $E^{(0)}$ is

$$
\begin{gathered}
E^{(0)}=-n-\Delta_{0}, \\
\Delta_{0}=n 2^{-n} \sum_{d=0}^{n}\left(\begin{array}{l}
n \\
d
\end{array}\right) \frac{B_{\perp}(n-2 d)}{n+\Delta_{0}-B_{\perp}(n-2 d)} .
\end{gathered}
$$


Here, $\Delta_{0}$ is the root of the above transcendental equation that satisfies the condition $\lim _{B_{\perp} \rightarrow 0} \Delta_{0}=0$. In general, the sum (37) is dominated by the region of values of $d$ such that $|d-n / 2|=\mathcal{O}\left(n^{1 / 2}\right)$ where the factor $2^{-n}\left(\begin{array}{l}n \\ d\end{array}\right)$ reaches its maximum $\sim n^{-1 / 2}$. In that region, we replace the binomial coefficient with a Gaussian function of $d$ and the summation with the integral over $d$. Taking the integral, we obtain $\Delta_{0}$ in a form of a series expansion in powers of $n^{-1}$ :

$$
\Delta_{0} \simeq-B_{\perp}^{2}-\frac{B_{\perp}^{4}}{n}+\mathcal{O}\left(n^{-2}\right) .
$$

A comparison between the exact and asymptotic solutions for $\Delta_{0}$ is shown in Fig. 6. For $B_{\perp} \ll n^{1 / 2}$, the overall shift of the energies of the marked states is negative and quadratic in $B_{\perp}$.

According to Eq. (21), all $M$ degenerate eigenstates $|\psi\rangle_{\beta}$ have the same weight $Q\left(E^{(0)}\right)=\sum_{j=1}^{M}\left|\psi_{\beta}^{(0)}\left(z_{j}\right)\right|^{2}$ on the marked state subspace. In the large $n$ limit, we have

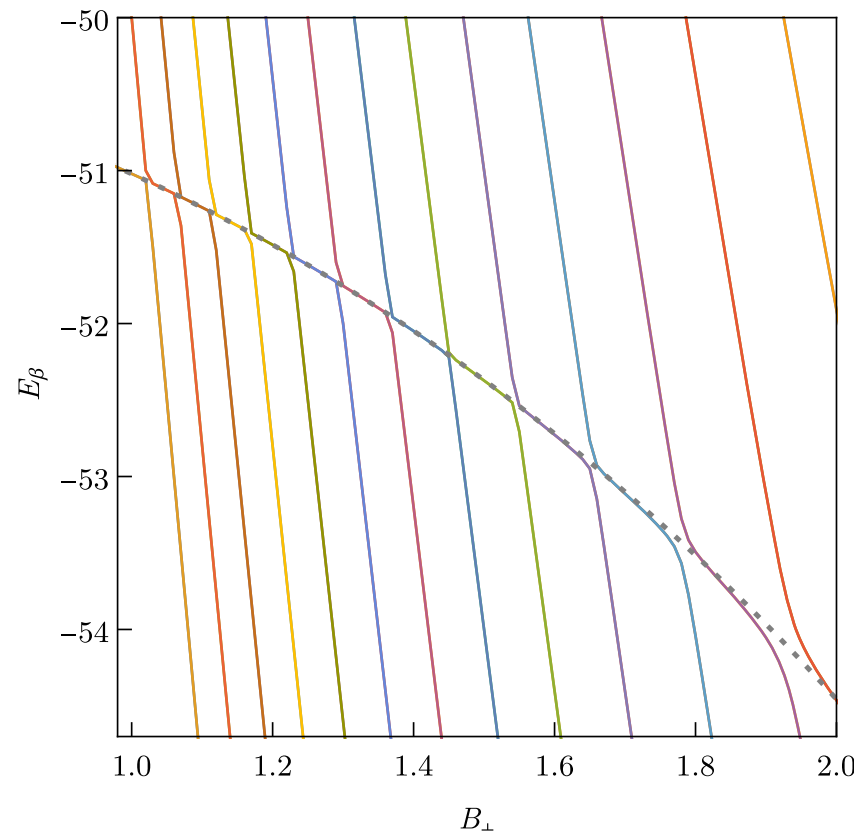

FIG. 6. Solid lines show the dependence on the transverse field $B_{\perp}$ of the eigenvalues $E_{\beta}$ of the nonlinear eigenvalue problem with Hamiltonian $\mathcal{H}(E)$ for the case of $n=50$ and $M=2$. The plot shows the repeated avoided crossing between the two systems of eigenvalues. One system (colored lines) corresponds to the eigenvalues of the transverse-field (driver) Hamiltonian $H_{D}=-B_{\perp} \sum_{k=0}^{n} \sigma_{x}^{k}$ in the limit $H_{\mathrm{cl}} \rightarrow 0$. The second system of eigenvalues corresponds to the energies of the two marked states in the limit $B_{\perp} \rightarrow 0$. The splitting of the eigenvalues is exponentially small in $n$ and not resolved in the plot. The asymptotic expressions (36) and (37) for the two eigenvalues $E_{1,2}^{(0)}=E^{(0)}$ neglecting the tunneling splitting and setting $\mathcal{E}\left(z_{j}\right)=-n$ for all $j \in[1, M]$ are shown with a dashed gray line.

$$
Q\left(E^{(0)}\right) \simeq 1-\frac{B_{\perp}^{2}}{n}+\mathcal{O}\left(B_{\perp}^{4} / n^{2}\right) .
$$

Under the condition $\propto B_{\perp}^{2} / n \ll 1$, the eigenstates are dominated by their projections on the marked state subspace. In the limit $n \rightarrow \infty$, they are asymptotically orthogonal to the computational basis states outside the IB. Such an orthogonality catastrophe cannot be obtained within the perturbative in $B_{\perp}$ approach such as the FSA.

The exact dependence of the weight $Q$ on transverse field $B_{\perp}$ is given in Fig. 7. The expression (39) is valid for $B_{\perp}$ away from their "resonant" values $B_{\perp, p} \simeq n /(n-2 p), \quad$ where the $M$-fold degenerate energy level "crosses" the eigenvalues of the driver Hamiltonian, $E^{(0)}=-B_{\perp}(n-2 p)$, for integer values of $p$, as shown in Fig. 6. The width of such resonance regions $\Delta B_{\perp, p} \propto 2^{-n / 2}\left(\begin{array}{l}n \\ p\end{array}\right)$ remains exponentially small in $n$ for $n / 2-p \gg n^{1 / 2}$.

In this study, we focus on the off-resonance case depicted in Fig. 1. One can see from Fig. 6 that $B_{\perp, p}$ increases with $p$ and so is the width of the resonance region. For $B_{\perp}$ parametrically large compared to unity, one needs to make sure that $n$ is also large enough so that the width of the resonance regions is small (cf. Fig. 8). Away from resonance, all $M$ impurity band eigenstates are well localized in the marked states subspace [cf. Eq. (39)].

In the spirit of the degenerate perturbation theory, there exists an effective Hamiltonian $\mathcal{H}$ that determines the correct zeroth-order eigenstates and removes the degeneracy of the energy levels:

$$
\mathcal{H}\left|\psi_{\beta}^{(0)}\right\rangle=E_{\beta}^{(1)}\left|\psi_{\beta}^{(0)}\right\rangle
$$

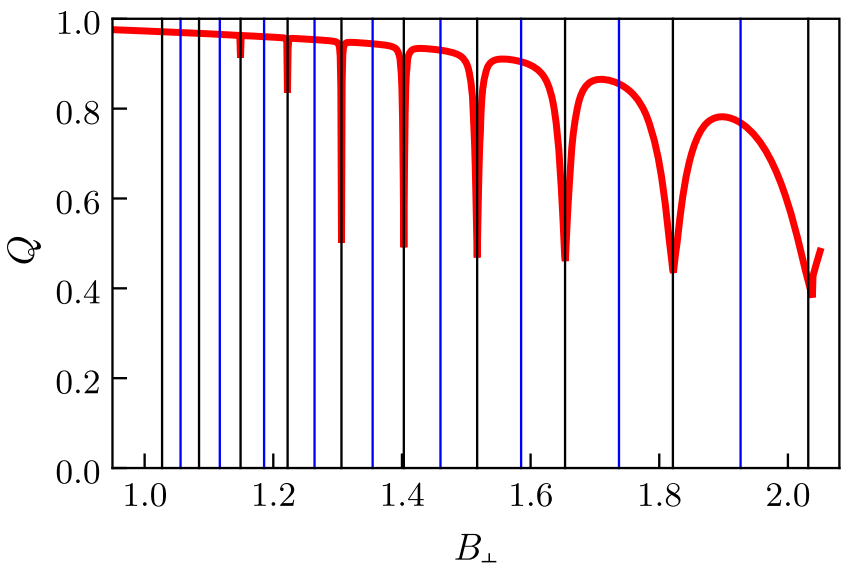

FIG. 7. The solid red line shows the dependence of the total weight $Q$ vs transverse field $B_{\perp}$ for $n=40$. Vertical black and blue lines, respectively, depict the locations of $p$-even and $p$-odd resonances $B_{\perp}=B_{\perp p}$ defined in the text. The total weight $Q$ undergoes sharp decreases in the vicinity of even resonances. For $p<5$, the resonance regions are so narrow that dips in $Q$ are not seen. The width of the regions grows steeply with $p$. 


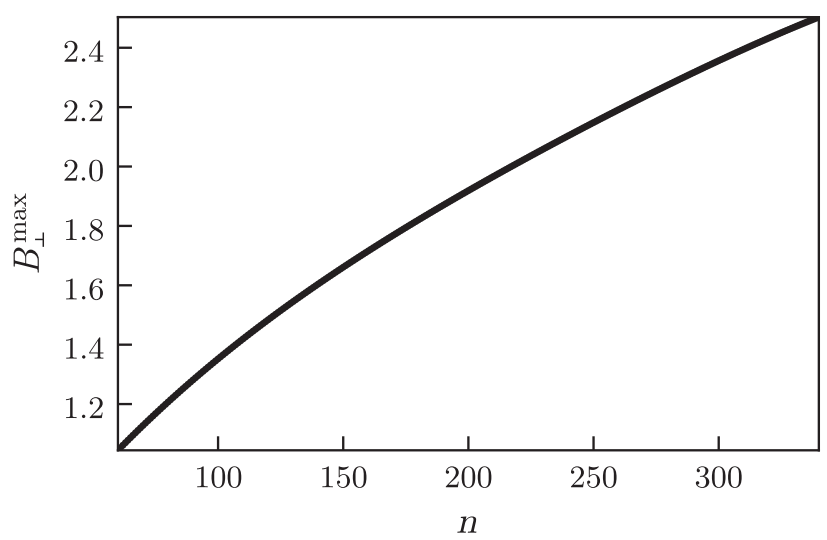

FIG. 8. Plot of the maximum value of the transverse field at midresonance point $B_{\perp}^{\max }$ as a function of $n$. We define $B_{\perp}^{\max }=\left(B_{\perp p}+B_{\perp p+1}\right) / 2$, where $B_{\perp, p} \simeq n /(n-2 p)$ satisfies the equation $E^{(0)}=-B_{\perp, k}(n-2 p)$ and the integer $p$ is equal to its maximum possible value $p=p_{\max }$ for which the weight factor $Q=Q\left(\left(B_{\perp p}+B_{\perp p+1}\right) / 2\right) \geq 0.98$.

Its matrix in the basis of the marked states has the form $\mathcal{H}_{i j}=\delta_{i j} \epsilon_{i}+n c\left(E^{(0)}, d_{i j}\right)$, where we neglect small nonimportant corrections (see Appendix $\mathrm{C}$ ). Using the expression for the coupling coefficient (33) given in Appendix B, [Eqs. (B23) and (B24)], we have

$$
\mathcal{H}_{i j}=\delta_{i j} \epsilon_{j}+\left(1-\delta_{i j}\right) \mathcal{V}_{i j} \sqrt{2} \sin \phi\left(d_{i j}\right)
$$

Here, $\phi(d) \equiv \phi\left(E^{(0)}, d\right)$ is a WKB phase shown in Fig. 5 that describes the oscillation of the matrix elements with the Hamming distance. Its explicit form is given in Appendix B, Eq. (B11), and also above in Eq. (35) for the case of large transverse fields. The amplitude $\mathcal{V}_{i j}$ equals

$\mathcal{V}_{i j} \equiv V\left(d_{i j}\right), \quad V(d)=\sqrt{A(d / n)} \frac{n^{5 / 4} e^{-n \theta\left(B_{\perp}\right)}}{\sqrt{\left(\begin{array}{l}n \\ d\end{array}\right)}}$,

where $i \neq j$ and the coefficient $A(\rho)$ equals [cf. Eq. (B24)]

$$
\begin{gathered}
A(\rho)=\sqrt{\frac{\pi}{32}} \frac{e^{-B_{\perp} \operatorname{arccoth} B_{\perp}}}{\left(B_{\perp}^{2}-1\right) v(\rho) \sin ^{4}[\phi(n / 2)]}, \\
v(\rho)=\left(1-\frac{(1-2 \rho)^{2}}{1-B_{\perp}^{-2}}\right)^{1 / 2} .
\end{gathered}
$$

It is independent of $n$ apart from the phase $\phi(n / 2)$, whose explicit form is

$$
\phi(n / 2)=\frac{\pi}{4}\left[n\left(1-B_{\perp}^{-1}\right)-B_{\perp}\right] .
$$

The function $\theta\left(B_{\perp}\right)$ is given in Eq. (34). Expanding Eq. (34) in the limit $B_{\perp} \gg 1$,

$$
\theta \simeq \frac{1}{4 B_{\perp}^{2}}+\frac{1}{24 B_{\perp}^{4}}+\frac{1}{60 B_{\perp}^{6}}+\cdots
$$

In that limit, $\theta \ll 1$. We note that, even for modest values of the transverse field, e.g., $B_{\perp} \simeq 1.46$ (corresponding to that in Fig. 4), the first term provides a good estimate to the value of $\theta \simeq 0.13$ (error 9\%). We refer to $\mathcal{H}$ in Eq. (41) as the IB Hamiltonian.

The form of the IB Hamiltonian (41) applies only to the region of oscillatory behavior $d_{i j} \in\left[n / 2-m_{0}, n / 2+m_{0}\right]$ of the coupling coefficients $c_{i j}(E)$ with Hamming distance $d_{i j}$, where $m_{0}$ is given in Eq. (32). This above condition for $d_{i j}$ is always satisfied in a typical row of the matrix $d_{i j}$ for the values of $M$ considered in this paper [see the discussion in Appendix G and Eq. (G32)].

\section{STATISTICAL ENSEMBLE OF THE IMPURITY BAND HAMILTONIANS}

Properties of the eigenstates and eigenvalues [Eq. (40)] of the IB Hamiltonian $\mathcal{H}$ [Eq. (41)] determine the population transfer within the impurity band and are, thus, of central interest for us in this study. They depend on the statistical ensemble of IB Hamiltonians. In the model considered in this paper, diagonal elements $\epsilon_{j}$ of $\mathcal{H}$ are selected at random, independently from each other and from the choice of the corresponding marked states $\left|z_{j}\right\rangle$. In the present discussion, we assume that the PDF $p(\epsilon)$ of $\epsilon_{j}$ is exponential bounded with the width $W$. The results do not depend on the particular form of $p(\epsilon)$. For the sake of specificity in calculations, we use the window function form

$$
p(\epsilon)=\theta(W / 2-|\epsilon|),
$$

where $\theta(x)$ is a Heaviside theta function. For the physical effects discussed in this paper to take place, the width $W$ needs to scale down exponentially with $n$ :

$$
\lim _{n \rightarrow \infty} \log \left(W^{1 / n}\right)=\mathcal{O}\left(n^{0}\right)
$$

\section{A. Off-diagonal matrix elements}

For fixed energies $\epsilon_{j}$, the matrix of the IB Hamiltonian $\mathcal{H}_{i j}$ is entirely determined by the symmetric matrix of Hamming distances $d_{i j}$ between the bit strings corresponding to the marked states. The set of $M$ bit strings is randomly sampled from the full set of all possible $2^{n}$ bit strings $\{0,1\}^{n}$ without replacement; see Appendix D. Elements of the matrix $d_{i j}$ above or below the main diagonal are considered independent from each other and taken from the binomial distribution $p_{d}$ : 


$$
p_{d}=\frac{1}{Z} 2^{-n}\left(\begin{array}{l}
n \\
d
\end{array}\right), \quad Z=\sum_{d=1}^{n} 2^{-n}\left(\begin{array}{l}
n \\
d
\end{array}\right)
$$

under condition $1 \ll M \ll 2^{n / 2}$. Then, for a given row of the matrix $M \times M$ of Hamming distances $d_{i j}$, the numbers of elements $M_{j}^{(d)}$ with $d_{i j}=d$ are samples from the multinomial distribution with mean values $\left\langle M_{j}^{(d)}\right\rangle=M p_{d}$ (see Fig. 9). According to Eqs. (41) and (33), the statistical ensemble of IB Hamiltonians (41) corresponds to that of symmetric random matrices whose associated graphs are fully connected and matrix elements are statistically independent.

As seen below, the spectral properties of $\mathcal{H}$ that are relevant for our study are determined by $\mathcal{V}_{i j}^{2}$ and not by the oscillatory factor in Eq. (41). Therefore, we are interested in the PDF of $\mathcal{V}_{i j}^{2}$ :

$$
P\left(\mathcal{V}_{i j}^{2}\right)=\sum_{d=1}^{n} p_{d} \delta\left[V^{2}(d)-\mathcal{V}_{i j}^{2}\right]
$$

where $i \neq j$.

\section{Typical and extreme values of the off-diagonal matrix elements $\mathcal{V}_{i j}$}

For a randomly chosen row of the matrix of Hamming distances $d_{i j}$, the most probable value (mean) of its elements equals $n / 2$. According to Eq. (42), the offdiagonal matrix elements $\mathcal{V}_{i j}$ decrease rapidly with the Hamming distance $d_{i j}$, reaching the minimum value at $d_{i j} \simeq n / 2$. Therefore, a typical minimum value of the matrix elements $\mathcal{V}_{i j}$ corresponds to a typical value overall. We estimate it using Eq. (42) and Stirling's approximation

$$
V_{\mathrm{typ}}=V(n / 2) \simeq\left(\frac{\pi A^{2}}{2}\right)^{1 / 4} n^{2} 2^{-n / 2} e^{-n \theta}
$$

where coefficient $A=A\left(E^{(0)}, 1 / 2\right)$ [Eq. (43)] is essentially $n$ independent between the resonances and $\theta$ is given in Eq. (34). The matrix elements $\mathcal{V}_{i j}$ that scale with $n$ as the typical value in Eq. (51) correspond to $\left|n / 2-d_{i j}\right|=\mathcal{O}(\sqrt{n})$.

We note that in Fig. 9 the plot points do not reach the boundaries of the interval $d=0, n$. In the matrix of Hamming distances $d_{i j}$, the typical smallest off-diagonal element in a randomly chosen row can be estimated as follows: $M p_{d_{\min }}=1$, where $p_{d}$ is binomial distribution (49):

$$
\min _{j \neq i, 1 \leq j \leq M} d_{i j} \sim d_{\min }, \quad M 2^{-n}\left(\begin{array}{c}
n \\
d_{\min }
\end{array}\right)=1 .
$$

Using Stirling's approximation for factorials in the limit $n \gg 1$, it is easy to show that the minimum Hamming distance in a row is extensive for $M=2^{\mu n}, \mu<1$.

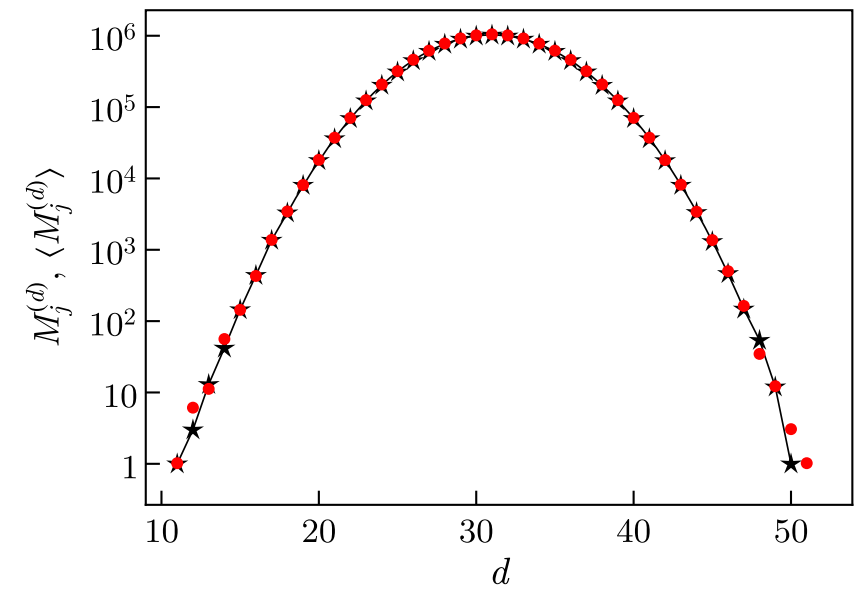

FIG. 9. Red points show the empirical probability distribution $M_{j}^{(d)}$ vs $d$ with $M_{j}^{(d)}=\sum_{j=1}^{M} \delta\left(d_{i j}-d\right)$. Here, $d_{i j}$ is a matrix of Hamming distances $d_{i j}$ between the set of $M$ randomly chosen $n$-bit strings (marked states), and $i$ is a randomly chosen marked state. The distribution corresponds to $M=10^{7}$ and $n=60$. Black stars connected by a black line show the samples $m_{d}$ from multinomial distribution with mean values $\left\langle M_{j}^{(d)}\right\rangle=M p_{d}$, where $p_{d}$ is binomial distribution (49).

The typical largest magnitude off-diagonal matrix element in a randomly chosen row of $\mathcal{V}_{i j}$ is equal to $V\left(d_{\text {min }}\right)$. Using Stirling's approximation in Eq. (42), we get

$$
\max _{j \neq i, 1 \leq j \leq M}\left|\mathcal{V}_{i j}\right| \sim M^{1 / 2} V_{\text {typ }}
$$

Using Eq. (51), one can see that the maximum off-diagonal matrix element in a randomly chosen row is still exponentially small in $n$.

Similarly, one can estimate the typical value of the absolute minimum $d_{\mathrm{abs} \text { min }}$ of a Hamming distance $d_{i j}$ between a pair of marked states. This distance remains extensive for $\mu<1, M=2^{\mu n}$. This distance corresponds to the overall largest in magnitude element of the matrix $\mathcal{V}_{i j}$ :

$$
\max _{1 \leq i<j \leq M}\left|\mathcal{V}_{i j}\right| \sim M V_{\text {typ }}
$$

Using Eq. (51), the largest element is exponentially small in $n$ provided that $\mu<1 / 2$, which corresponds to the condition of statistical independence of the elements of $\mathcal{V}_{i j}$. A tight bound for the maximum eigenvalues of $H$ can be obtained using the Gerschgorin circle theorem [58]; see Appendix E.

\section{B. Heavy tails}

It can be shown that the variance of $\mathcal{H}_{i j}$ is not a good statistical characteristic of its PDF and is dominated by the extremely rare atypical instances of the ensemble (see details in Appendix F). We observe that the relationship 
between the typical matrix element (51), maximum matrix element in a randomly chosen row of $\mathcal{V}_{i j}$ (53), and the largest element of $\mathcal{V}_{i j}$ overall (54) form a strong hierarchy that is a characteristic of the ensemble of dense matrices with broad nonexponential distribution of matrix elements (Levy matrices) [33]. The form of the hierarchy [37] suggests (up to a logarithmic factors) the following asymptotic behavior at the tail of the PDF of the matrix elements:

$$
\operatorname{PDF}\left(\mathcal{V}_{i j}^{2}\right) \propto\left|\mathcal{V}_{i j}\right|^{-2}
$$

for $\left|\mathcal{V}_{i j}\right| \gg V_{\text {typ }}$.

We build on the above observation and obtain the explicit form of the PDF of the matrix elements $P\left(\mathcal{V}_{i j}^{2}\right)$ [Eq. (50)], including its tails. In the asymptotic limit of large $n \gg 1$, we consider $n$ to be a continuous variable (the validity of this approximation is justified below). We replace the summation over $d$ in Eq. (50) by an integral and Kronecker delta $\delta(x)$ by Dirac delta

$$
P\left(\mathcal{V}_{i j}^{2}\right) \simeq \int_{0}^{n} p_{x} \delta\left[V^{2}(x)-\mathcal{V}_{i j}^{2}\right] d x
$$

This expression is obtained using the analytical continuation of the binomial distribution $p_{d}$ [Eq. (49)] from the integer domain $d \in(0, n)$ onto the interval of a real axis $x \in(0, n)$ in terms of the Beta function and the resulting identity $\int_{0}^{n} d x p_{x}=1$ (see Appendix $\mathrm{G}$ for details).

In what follows, we study the rescaled quantities

$$
w_{i j}=\frac{V_{i j}^{2}}{V_{\mathrm{typ}}^{2}} \equiv \frac{V^{2}\left(d_{i j}\right)}{V_{\mathrm{typ}}^{2}},
$$

where $i \neq j$ and $V_{\text {typ }}$ is given in Eq. (51). We apply Stirling's approximation for the binomial coefficient in Eqs. (42) and (49) and obtain asymptotic expressions for $V^{2}(d)$ and $p_{d}$, respectively. Plugging them into Eq. (55) and taking the integral there, we can obtain the PDF

$$
g\left(w_{i j}\right)=V_{\mathrm{typ}}^{2} P\left(V_{\mathrm{typ}}^{2} w_{i j}\right),
$$

whose form is given in Appendix G, Eqs. (G14) and (G15).

The following assumption is applied throughout this paper:

$$
M=2^{\mu n}, \quad \mu \ll 1 .
$$

According to Eqs. (42), (52), and (G5), a typical largest element in a randomly chosen row of the matrix $w_{i j}$ is $\sim M$. Therefore, based on Eq. (58), the following condition is satisfied in a randomly chosen row of $w_{i j}$ :

$$
\frac{1}{n} \log _{2} w_{i j} \ll 1 \quad\left(1 \leq w_{i j} \lesssim M\right) .
$$

Under this condition, the PDF of $w_{i j}$ takes a particularly simple form, $g(w) \simeq g_{\infty}(w)$ :

$$
g_{\infty}(w)=\frac{1}{w^{2} \sqrt{\pi \log w}}, \quad w \in(1, \infty)
$$

with normalization condition $\int_{1}^{\infty} g_{\infty}(w) d w=1$. Details of the derivation are given in Appendix G.

The above analysis assumes the scaling behavior (42) of $\mathcal{V}_{i j}$ with $d_{i j}$ that requires $\left|n / 2-d_{i j}\right|<m_{0}$ with $m_{0}$ given in Eq. (32). As shown in Appendix G, this condition is always satisfied for a typical row of $d_{i j}$ provided the constraint (58) on the values of $M$.

\section{PBLMs}

The problem of population transfer is reduced to the analysis of the described-above ensemble of real symmetric $M \times M$ matrices $\mathcal{H}_{i j}$ of the down-folded IB Hamiltonian (41). The matrices $\mathcal{H}_{i j}$ form an ensemble of PBLMs, a generalization of Levy matrices actively studied in the literature (cf. e.g., Refs. [33-35,37]). Unlike Levy matrices, PBLMs have a new control parameter: the ratio of typical diagonal to off-diagonal matrix elements $W / V_{\text {typ }}$ that controls the preferential basis (computation basis). This distinction is analogous to that between the Gaussian orthogonal ensemble and the Gaussian ensemble with broken $S U(N)$ symmetry, the RP model [51].

Recent studies of the RP ensemble [21] demonstrate two localization transitions that occur with a varying parameter that controls the relative weight of the diagonal and offdiagonal matrix elements. One of them is the Anderson transition from localized to the extended states that are nonergodic and possess distinct multifractal features. These states and the corresponding eigenvalues are organized in minibands so that the states within the same miniband mostly share the same support over basis states. The spectral width of the minibands is polynomially small (in $M$ ) compared to $W$. The second transition is from the extended nonergodic states to the extended ergodic states similar to the eigenstates of the Gaussian orthogonal ensemble. We demonstrate analogous behavior in the IB model and analyze the population transfer in the nonergodic regime.

\section{NUMERICAL SIMULATIONS: MINIBANDS OF NONERGODIC DELOCALIZED STATES}

In this section, we report an exact diagonalization analysis of both the eigenvector statistics and the dynamical eigenstate correlator. Instead of the sparse $2^{n} \times 2^{n}$ Hamiltonian Eq. (1), it is efficient to diagonalize the dense $M \times M$ matrices obtained by down-folding the 
Hamiltonian into the marked state subspace. This diagonalization allows access to systems of $n=200$ qubits, reducing the finite size effects. The down-folded matrix Hamiltonian ensemble is constructed as in Sec. VI:

$$
\mathcal{H}_{i i}=\epsilon_{i}, \quad \mathcal{H}_{i j}=n c\left(E^{(0)}, d_{i j}\right),
$$

where the diagonal elements $\epsilon_{m}$ are distributed uniformly in the energy window $[-n-W / 2,-n+W / 2]$ and the off-diagonal elements are constructed by sampling Hamming distances between uniformly random bit strings of length $n$ and using Eq. (23) with $E=E^{(0)}$ determined from Eqs. (36) and (37).

We introduce the scaling of the width of the distribution of $\epsilon_{m}$ with the matrix size $M$ :

$$
W=\lambda M^{\gamma / 2} V_{\mathrm{typ}}
$$

where $\gamma$ is a real non-negative parameter that controls the scaling of the typical diagonal to off-diagonal matrix element $V_{\text {typ }}$ given in Eq. (51) and $\lambda$ is an auxiliary constant of the order of one.

\section{A. Eigenvector statistics}

We define the inverse participation ratios (IPRs) $I_{q}$ and the entropy $H^{z}$ as

$$
\begin{gathered}
I_{q}=\sum_{i}\left|\left\langle\psi_{\beta} \mid i\right\rangle\right|^{2 q}, \\
H^{z}=-\sum_{i}\left|\left\langle\psi_{\beta} \mid i\right\rangle\right|^{2} \ln \left|\left\langle\psi_{\beta} \mid i\right\rangle\right|^{2},
\end{gathered}
$$

respectively, where $\psi_{\beta}$ denotes an eigenstate with eigenvalue $E_{\beta}$. IPR $I_{2}$ is the second moment of the wave function probability distribution $\left|\left\langle\psi_{\beta} \mid i\right\rangle\right|^{2}$ in the computational basis (bit strings) $|i\rangle$. The entropy $H^{z}$ characterizes the support set of an eigenstate in the computational basis [59], i.e., the subset of bit strings where the probabilities $\left|\left\langle\psi_{\beta} \mid i\right\rangle\right|^{2}$ are concentrated.

Figure 10 shows the participation ratio $I_{2}$ as a function of the ratio of mean level spacing $\delta \epsilon$ to the typical matrix element $V_{\text {typ }}$, a measure of the number of states in resonance with a typical classical level $\epsilon_{i}$. The regime $\delta \epsilon \gg V_{\text {typ }}$ corresponds to the localized phase, where the eigenstates have significant weight on a small number of bit strings that are close to each other in Hamming distance. In this regime, $I_{2} \sim 1$ and is system size independent. In our model, marked states are separated by Hamming distance $d \approx n / 2+\mathcal{O}(\sqrt{n})$ with a high probability, and, therefore, most localized states have sharp peaks at exactly one bit string; hence, $I_{2} \approx 1$. As the ratio $\delta \epsilon / V_{\text {typ }}$ decreases, $I_{2}$ becomes system size dependent. Figure 11 indicates that the combination $I_{2} M / 3 \sim 1$ becomes system

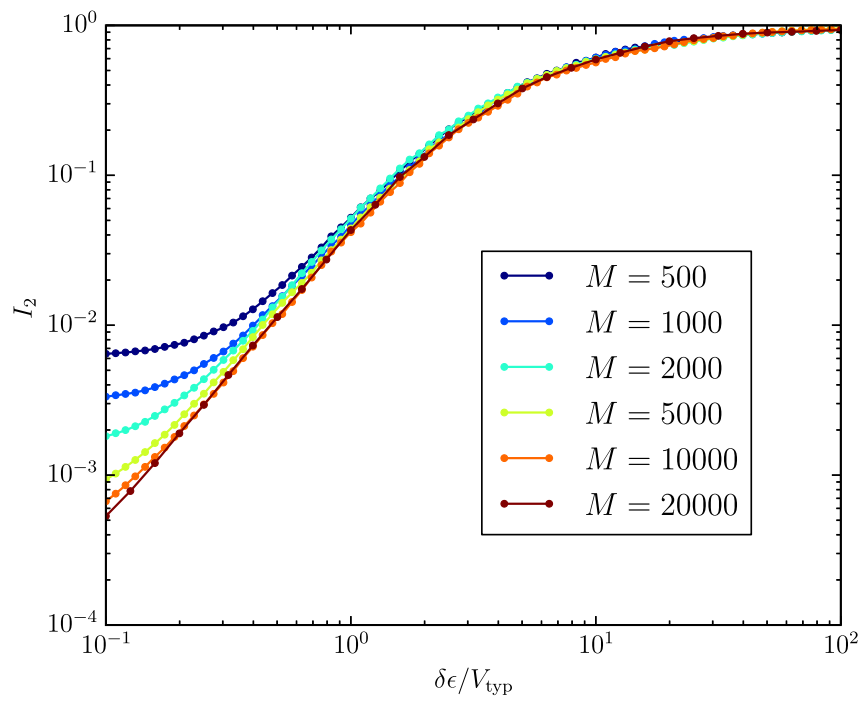

FIG. 10. The inverse participation ratio $I_{2}=\sum_{i}\left|\left\langle i \mid \psi_{\beta}\right\rangle\right|^{4}$ as a function of the average classical (at a vanishing transverse field) energy level spacing $\delta \epsilon$ in units of the typical coupling $V_{\text {typ }}$ for different numbers $M$ of states in the impurity band. We see that for $\delta \epsilon / V_{\text {typ }} \geq 1$ the eigenstates become localized and $I_{2} \rightarrow 1$ independent of $M$, indicative of eigenstates localized on single bit string each.

size independent as the level spacing becomes smaller than the typical matrix element, characteristic of the delocalized regime, where the wave function amplitude spreads over $\mathcal{O}(M)$ bit strings, $\left|\left\langle\psi_{\beta} \mid i\right\rangle\right|^{2} \sim 1 / M$. The saturation value of $I_{2} M \sim 3$ is consistent with approach to Porter-Thomas distribution of the wave function amplitudes. Both Figs. 10 and 11 show a wide intermediate region between the localized and ergodic phases where nonergodic dynamics is expected. This intermediate regime becomes apparent in Fig. 12, where we introduce the multifractal dimensions $D_{q}$ and $D_{1}$, which determine the scaling of $I_{q}$ and $H^{z}$ with $M$, respectively:

$$
\begin{gathered}
\ln I_{q}(M)=-D_{q}(q-1) \ln M+c_{q}, \\
H^{z}(M)=D_{1} \ln M+c_{1},
\end{gathered}
$$

where $c_{q}$ is a $q$-dependent fitting parameter. The extracted dimensions shown in Fig. 12 as a function of the parameter $\gamma$ vary continuously between $D_{q}=1$ in the ergodic phase $\gamma \leq 1$ and $D_{q}=0$ in the localized phase $\gamma \geq 2$, with $1<$ $\gamma<2$ corresponding to a nonergodic regime for $q=1,2$.

\section{B. Eigenstate overlap correlator for nonergodic minibands}

Population transfer dynamics in the nonergodic regime can be characterized by the survival probability; see Sec. II. The Fourier transform of the survival probability for a given initial marked state $i$ is given by 


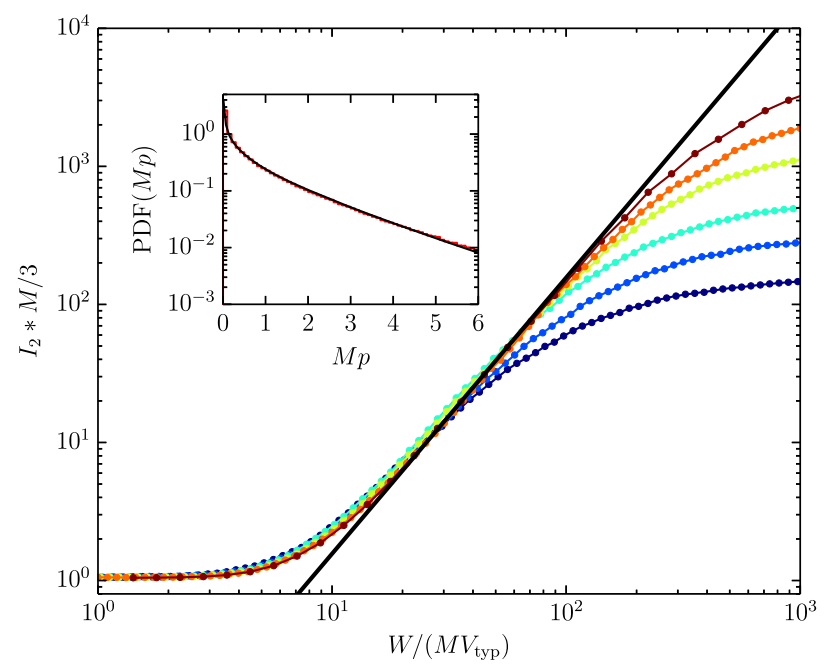

FIG. 11. The rescaled inverse participation ratio $I_{2} M / 3$ as a function of the rescaled impurity band width $W /\left(M V_{\text {typ }}\right)$ for different numbers $M$ of states in the impurity band. We see that in the ergodic regime, $W /\left(M V_{\text {typ }}\right) \leq 1$, we have $I_{2} M / 3=1$, corresponding to the orthogonal Porter-Thomas distribution of states in the impurity band. The inset shows the numerical probability distribution of normalized probabilities $M p$ for an eigenstate over computational states $z$ in the ergodic regime in black and the analytical orthogonal Porter-Thomas distribution in red. Qualitative arguments in Sec. VIII suggest that in the nonergodic delocalized regime $I_{2} M / 3 \propto\left[W /\left(M V_{\text {typ }}\right)\right]^{2}$. The black line is proportional to $\left[W /\left(M V_{\text {typ }}\right)\right]^{2}$, and we see that $I_{2} M / 3$ aligns with this quantity as long as we do not enter the localized regime $\delta \epsilon / V_{\text {typ }} \geq 1$; see Fig. 10 .

$$
\begin{aligned}
p_{i}(\omega) & =\operatorname{Re} \int_{0}^{\infty} d t e^{i \omega t}|\langle i \mid \psi(t)\rangle|^{2} \\
& =\pi \sum_{\beta, \beta^{\prime}}\left|\left\langle i \mid \psi_{\beta}\right\rangle\right|^{2}\left|\left\langle\psi_{\beta^{\prime}} \mid i\right\rangle\right|^{2} \delta\left(E_{\beta}-E_{\beta^{\prime}}-\omega\right) .
\end{aligned}
$$

Note that the limit $\omega \rightarrow 0$ gives the inverse participation ratio of a given bit string in the basis of eigenstates:

$$
p_{i}(0)=\pi \sum_{\beta}\left|\left\langle i \mid \psi_{\beta}\right\rangle\right|^{4}
$$

The average of $p_{i}(\omega)$ over the initial state is related to the overlap correlation function $K(\omega)$ defined by [21]

$$
\begin{aligned}
K(\omega) & \equiv \frac{1}{M} \sum_{i, \beta, \beta^{\prime}}\left|\left\langle\psi_{\beta} \mid i\right\rangle\right|^{2}\left|\left\langle\psi_{\beta^{\prime}} \mid i\right\rangle\right|^{2} \delta\left(E_{\beta}-E_{\beta^{\prime}}-\omega\right) \\
& =\frac{1}{\pi M} \sum_{i} p_{i}(\omega) .
\end{aligned}
$$

The fractal dimension extracted from the scaling of $K(0)$ with $M$ is shown in Fig. 12, and it follows closely those extracted from the IPR in the computational basis.

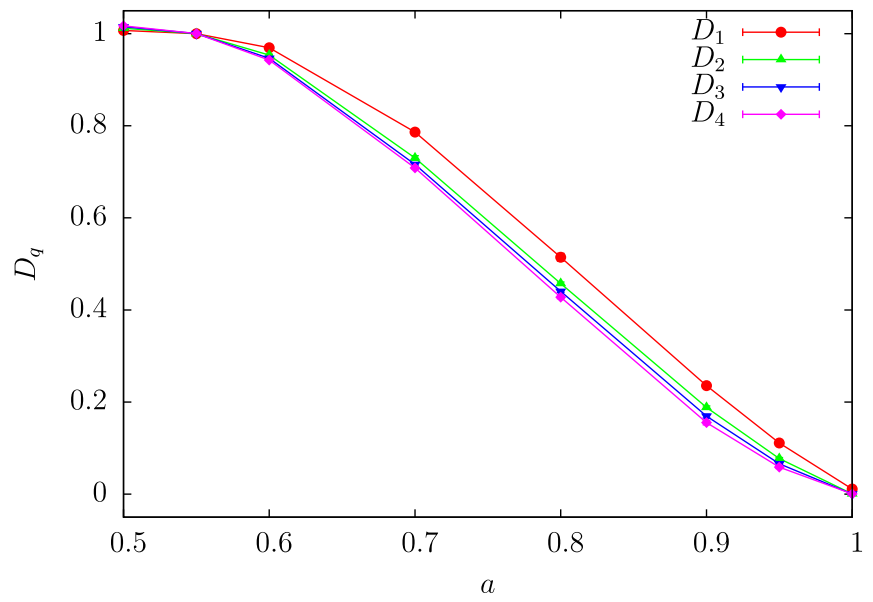

FIG. 12. The multifractal dimensions $D_{1}$ [defined in Eq. (66)] and $D_{2}$ [defined in Eq. (65)] as functions of $\gamma$ for the ensemble of IB Hamiltonians with the dispersion of classical energies $W=\lambda V_{\text {typ }} M^{\gamma / 2}$, with $\lambda=3.3$. All the multifractal dimensions $D_{q}$ approach 1 in the ergodic regime $(\gamma=1)$ and 0 in the localized regime $(\gamma=2)$. The difference between $D_{1}$ and $D_{2}$ is also likely due to finite size effects. We also extract a scaling exponent from the dynamical correlator [see Eqs. (68) and (69)]. The dot-dashed line corresponds to the analytical value in the Rosenzweig-Porter limit given by Eq. (72).

The collapse of the plots in Fig. 13 is achieved when the frequency is rescaled by the characteristic energy:

$$
\Gamma_{\varepsilon}=\Gamma_{\mathrm{typ}} M^{\varepsilon}, \quad \Gamma \propto V_{\mathrm{typ}} M^{1-\gamma / 2}(\log M)^{1 / 2},
$$

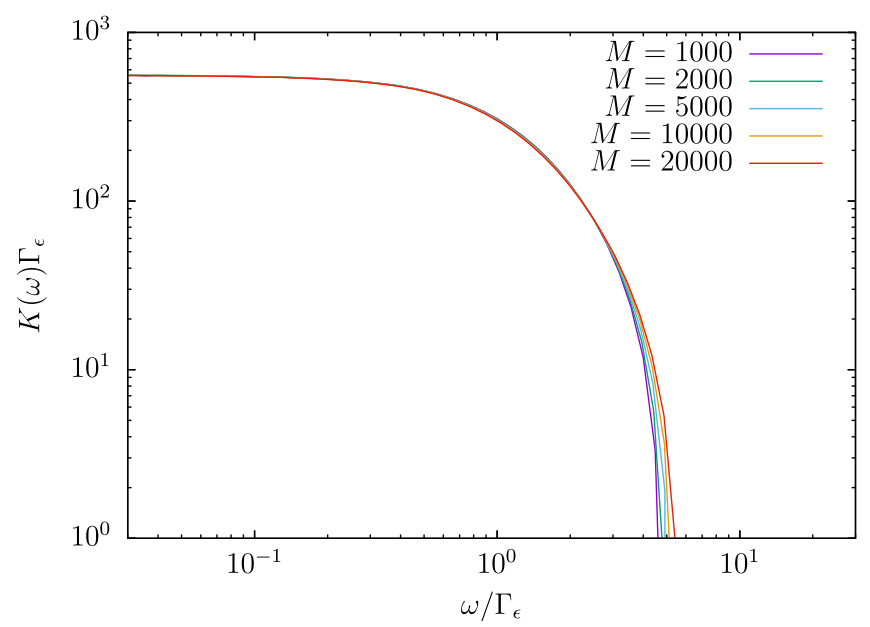

FIG. 13. We plot the rescaled overlap correlation function $K(\omega) \Gamma_{\varepsilon}$ vs $\omega / \Gamma_{\varepsilon}$, where $\Gamma_{\varepsilon}=\Gamma_{\text {typ }} M^{\varepsilon}$ and $\Gamma_{\text {typ }}=2 \Sigma_{\text {typ }}^{\prime \prime}$ is the typical miniband width and $\Sigma_{\text {typ }}^{\prime \prime} \propto V_{\text {typ }} M^{1-\gamma / 2}(\log M)^{1 / 2}$ [Eq. (128)]. Different curves correspond to different values of $M$ and collapse well with $\varepsilon=0.05$. We use the ensemble of IB Hamiltonians with a dispersion of classical energies $W=\lambda V_{\text {typ }} M^{\gamma / 2}$, with $\gamma=1.2$ and $\lambda=3.3$. 
with a fitting parameter $\varepsilon \ll 1$. The correlator $K(\omega)$ is constant for a range of energy differences $\omega<\Gamma_{\varepsilon}$ and decays quickly $\propto \omega^{-2}$ as $\omega>\Gamma_{\varepsilon}$. This result can be interpreted in terms of the formation of nonergodic minibands of eigenstates that share support in the computation basis: For an average bit string, there is a range of eigenenergies $E_{\beta}$ within a width $\Gamma_{\varepsilon}$ around a bit-stringdependent value $\epsilon_{j}$ where the eigenfunction overlaps with $z_{j}$ are relatively large, whereas for a larger energy difference the correlation decays quickly below the value corresponding to uncorrelated case $K(\omega)<1 / M$; i.e., the amplitudes repel each other. The relation between the survival probability and eigenfunction overlap correlator [Eq. (69)] suggests that the characteristic population transfer is given by the inverse of the characteristic energy scale of the miniband width $\Gamma_{\varepsilon}$, the range of energy eigenstates with a significant amplitude at the given bit string. The auxiliary fitting parameter takes a small value of $\varepsilon=0.05$, indicating only a small deviation from $\Gamma_{\text {typ }}$ most likely due to finite size effects. In Appendix M, we show the results of a direct simulation of dynamics of the model in the course of the PT protocol and confirm the scaling of the PT time.

\section{Discussion of numerical results}

The size of the matrix of marked states used in exact diagonalization $M \leq 20000$ is a small fraction of the size of the total Hilbert space Hamiltonian $2^{n} \times 2^{n}$ with $n=200$. For such a small sample, the distribution of Hamming distances $d_{i j}$ between marked states is dominated by $\left|d_{i j}-n / 2\right| \sim \mathcal{O}(\sqrt{n})$. In this regime, the square of the off-diagonal matrix element (see Sec. IV) has an approximately Gaussian dependence on $d_{i j}$ [cf. Eqs. (42) and (51)]:

$$
\mathcal{H}_{i j}^{2} \approx V_{\text {typ }}^{2} \exp \left[\frac{2}{n}\left(d_{i j}-\frac{n}{2}\right)^{2}\right],
$$

and the probability to find a pair of bit strings at a smaller distance $d_{i j}$ is strongly suppressed. The sign of $\mathcal{H}_{i j}$ rapidly fluctuates as a function of $d_{i j}$, resulting in a negligible average $\left\langle\mathcal{H}_{i j}(d)\right\rangle \sim \mathcal{O}\left(2^{-n}\right)$. The distribution of off-diagonal matrix elements in Eq. (71) is non-Gaussian and, instead, has a heavy tail that cannot be fully characterized by the variance alone; see Sec. VIB and Appendix F, where we introduce the class of preferred basis Levy matrices and derive the asymptotic form of the distribution of matrix elements. For numerically accessible matrix sizes $M$, we expect the deviation from the Gaussian distribution in the observables to be very small.

The eigenstate statistics and the respective fractal dimensions for the model Eq. (71) can be calculated using the strong disorder perturbation theory. The calculation proceeds similarly to that in Ref. [21], resulting in

$$
D_{1}=D_{2}=2-\gamma .
$$

A comparison of the approximate Eq. (72) with numerical results is shown in Fig. 12 as the dot-dashed line. It appears that the $D_{1}$ and $D_{2}$ do not quite coincide with each other nor with Eq. (72), which may be due to finite size effects.

It is instructive to draw an analogy between characteristics of the PBLMs and that of the RP model from random matrix theory; see Refs. [21,51], and references therein, where the matrix elements are given by a Gaussian random variable with zero mean and variance for diagonal and all off-diagonal matrix elements set $\left\langle\mathcal{H}_{i i}^{2}\right\rangle=1$ and $\left\langle\mathcal{H}_{i j}^{2}\right\rangle \propto M^{\gamma}$. Transition points between localized, delocalized, and nonergodic delocalized regimes as well as perturbative expressions for fractal dimensions [Eq. (72)] are consistent in the two models. The dynamical correlator also shows similar behavior indicative of the formation of minibands of nonergodic eigenstates with the leading exponent $1-\gamma / 2$ in the scaling of the population transfer time with $M$ coinciding in the two models. The prefactor $(\log M)^{1 / 2}$, however, is affected by the heavy tail of the distribution of the matrix elements and needs to be calculated analytically. It is difficult to extract it accurately from the numerical simulations due to the finite size effects.

\section{BORN APPROXIMATION FOR THE TRANSITION RATES}

In this section, we develop a simple picture relying on the Fermi golden rule (FGR) to study the rates of population transfer away from a given marked state to a set of other marked states inside the same miniband. Assume that the system is initially prepared at a randomly chosen marked state $\left|z_{j}\right\rangle$. The probability amplitude to remain in the initial state $\left|z_{j}\right\rangle$ equals

$$
\psi\left(z_{j}, t\right)=\sum_{\beta} \psi_{\beta}^{2}\left(z_{j}\right) e^{-i E_{\beta} t},
$$

where $|\psi(t)\rangle$ evolves with the IB Hamiltonian $\mathcal{H}(41)$ and $\mathcal{H}\left|\psi_{\beta}\right\rangle=E_{\beta}\left|\psi_{\beta}\right\rangle$. If the eigenstates dominantly coupled to the marked state $\left|z_{j}\right\rangle$ are extended, then the amplitude $\psi\left(z_{j}, t\right)$ undergoes a decay in time.

Here, we calculate $\psi\left(z_{j}, t\right)$ using a simple effective FanoAnderson model for the decay of a discrete state into a continuum [60]. This model captures the Born approximation for the ensemble of Hamiltonians introduced in Sec. VI. The model Hamiltonian $\tilde{\mathcal{H}}$ is obtained from the IB Hamiltonian $\mathcal{H}$ (41) by zeroing out all off-diagonal matrix elements except those in the $j$ th column and the $j$ th row connecting state $\left|z_{j}\right\rangle$ to the rest of the marked states. The Hamiltonian $\tilde{\mathcal{H}}$ has the form 


$$
\begin{aligned}
\tilde{\mathcal{H}}= & \epsilon_{j}\left|z_{j}\right\rangle\left\langle z_{j}\left|+\sum_{m \neq j}\left(\epsilon_{m}-i \eta\right)\right| z_{m}\right\rangle\left\langle z_{m}\right| \\
& +\sum_{m \neq j} \mathcal{H}_{j m}\left(\left|z_{j}\right\rangle\left\langle z_{m}|+| z_{m}\right\rangle\left\langle z_{j}\right|\right),
\end{aligned}
$$

where the summation is over $m \in[1 \ldots M], m \neq j$. We consider the dynamics on a timescale when the population of the state $\left|z_{j}\right\rangle$ decays into the other states and introduce a small imaginary part $-i \eta$ to their energies. It is assumed to be much bigger than the typical energy spacing $\eta \gg$ $\delta \epsilon=W / M$ but smaller than the timescale on which the decay takes place. We introduce the parameterization similar to that in Sec. VII for the distribution of energies $\epsilon_{j}$ :

$$
W=\lambda V_{\mathrm{typ}} M^{\gamma / 2}
$$

where $\lambda$ is a (redundant) number of the order of $\mathcal{O}\left(M^{0}\right)$.

The amplitude $\psi\left(t, z_{j}\right)$ has a well-known form [60]:

$$
\psi\left(z_{j}, t\right)=\int_{-\infty}^{\infty} \frac{d z}{\pi} \frac{\Sigma_{j}^{\prime \prime}(z) \exp (-i z t)}{\left[z-\Sigma_{j}^{\prime}(z)-\epsilon_{j}\right]^{2}+\left[\Sigma_{j}^{\prime \prime}(z)\right]^{2}},
$$

where we used a shorthand notation

$$
\Sigma_{j}(z)=\Sigma_{j}^{\prime}(z)-i \Sigma_{j}^{\prime \prime}(z)
$$

for real and imaginary parts of self-energy of the marked state $\left|z_{j}\right\rangle$,

$$
\Sigma_{j}(z)=\sum_{m \neq j} \frac{\mathcal{H}_{j m}^{2}}{z-\epsilon_{m}+i \eta},
$$

and we keep $z$ real. Calculating the above integral to the leading order in $\mathcal{H}_{j m}(j \neq m)$, we get

$$
\psi\left(z_{j}, t\right) \simeq \exp \left[-i\left(\epsilon_{j}+\Delta \epsilon_{j}\right) t-\frac{1}{2} \Gamma_{j} t\right],
$$

where

$$
\Delta \epsilon_{j} \simeq \Sigma_{j}^{\prime}\left(\epsilon_{j}\right), \quad \Gamma_{j} \simeq 2 \Sigma_{j}^{\prime \prime}\left(\epsilon_{j}\right) .
$$

The quantity $\Gamma_{j}$ above is the total decay rate of the state $\left|z_{j}\right\rangle$, which is twice the imaginary part of the self-energy $\Sigma_{j}^{\prime \prime}$. The latter equals to the "width" of the level $\epsilon_{j}$ due to the decay. Expressions (79) and (80) correspond to a wellknown Born approximation for the self-energy $\Sigma_{j}^{\prime \prime}$. Using Eq. (78), we get

$$
\Sigma_{j}^{\prime \prime}=\pi \sum_{m \in[1 \ldots M] / j} \mathcal{H}_{m j}^{2} \delta\left(\epsilon_{j}-\epsilon_{m}, \eta\right)
$$

where we defined a function:

$$
\delta(\epsilon, \eta) \equiv \frac{1}{\pi} \frac{\eta}{\epsilon^{2}+\eta^{2}}
$$

The matrix elements $\mathcal{H}_{m j}^{2}$ [see Eqs. (41) and (42)] depend only on the Hamming distance $d_{m j}$. The dominant contribution into the sum (81) comes from the transitions to the states with $\left|\epsilon_{j}-\epsilon_{m}\right| \lesssim \eta$. If the number of such states is large, the sum can be replaced by the integral corresponding to the approximation where the Lorentzian $\delta\left(\epsilon_{j}-\epsilon_{m}, \eta\right) \approx\left\langle\delta\left(\epsilon_{j}-\epsilon_{m}, \eta\right)\right\rangle$ is replaced with its average over realizations of $\epsilon_{m}$. The average is independent of $\eta \ll W$, which, therefore, drops out from the PDF of the transition rate $\Gamma$ and the resulting level width $\Sigma^{\prime \prime}=\Gamma / 2$. This case corresponds to the leading-order Born approximation described in Sec. VIII A.

A more accurate treatment of $\delta\left(\epsilon_{j}-\epsilon_{m}, \eta\right)$ as a random variable results in the form of the PDF of $\Gamma$ (and $\Sigma^{\prime \prime}$ ) being explicitly dependent on $\eta$. The physical meaning of $\eta$ is the decay rate at the "children" sites $\epsilon_{m}, m \neq j$, which gives rise to the width $\Sigma^{\prime \prime}$ or the energy level $\epsilon_{j}$ at the parent site. In a large system, the statistics of the decay rate for children and parents are expected to be the same. The crude approximation that captures this effect is obtained by substituting $\eta$ with a typical value of $\Sigma^{\prime \prime}$. This approximation corresponds to self-consistent Born approximation described in Sec. XI A 2. It gives rise to a more accurate expression for the PDF of $\Sigma^{\prime \prime}$ (and $\Gamma$ ) whose shape is rescaled compared to the leading-order Born. A systematic analysis is given by the cavity method described in Secs. X and XI.

\section{A. Leading-order Born approximation}

We can break down the decay rate $\Gamma_{j}=2 \Sigma_{j}^{\prime \prime}$ into a sum over different decay channels:

$$
\Sigma_{j}^{\prime \prime}=\pi \sum_{d=1}^{n} V^{2}(d)[1-\cos 2 \phi(d)] \varrho_{\eta}^{j}(d),
$$

where each term in the sum corresponds to the transition rate from the initial state $\left|z_{j}\right\rangle$ into the subset of the marked states on a given Hamming distance $d$ from $\left|z_{j}\right\rangle$ (see Fig. 14). The factor $Q_{\eta}^{j}(d)$ in Eq. (83) is a spectral density of the marked states located at a distance $d$ from the state $\left|z_{j}\right\rangle$ within the window of energies $\eta$ around $\epsilon_{j}$ :

$$
\varrho_{\eta}^{j}(d)=\sum_{m \neq j} \delta\left(\epsilon_{j}-\epsilon_{m}, \eta\right) \Delta\left(d-d_{j m}\right),
$$

where $\Delta(d)$ is a Kronecker delta and $\delta(\epsilon, \eta)$ is defined in Eq. (82).

We denote as $M_{j}^{(d)}$ the number of marked states that are separated by a Hamming distance $d$ from the state $\left|z_{j}\right\rangle$ [number of terms in the sum (84)]: 


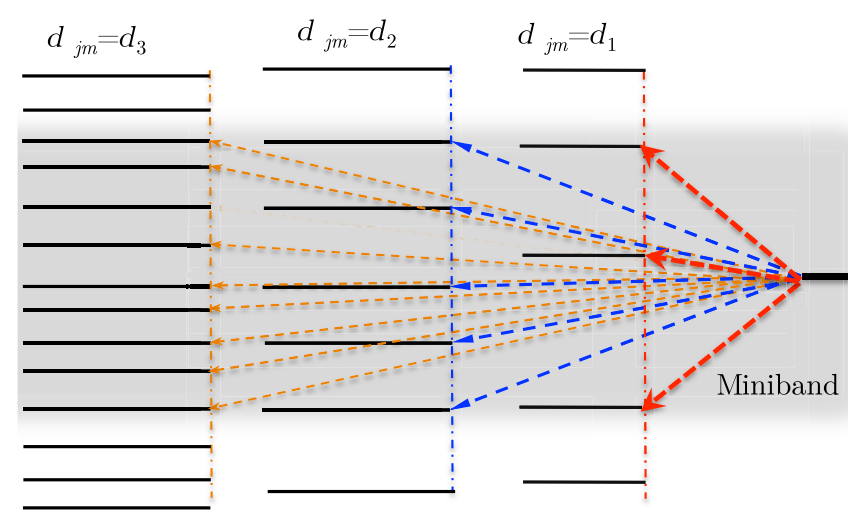

FIG. 14. Cartoon of the energies of the marked states $\epsilon_{m}$ within the impurity band. Energy levels are shown with solid black lines forming groups arranged vertically. All states $\left|z_{m}\right\rangle$ within one group lie at the same Hamming distance $d_{j m}=d$ from a given state $\left|z_{j}\right\rangle$ with $d$ increasing from right to left. The energy level $\epsilon_{j}$ is depicted at the right side of the figure with a thick black line. Arrows depict the transitions away from the initial state $|\psi(0)\rangle=$ $\left|z_{j}\right\rangle$ into the marked states $\left|z_{m}\right\rangle$ whose energy levels lie inside the miniband of the width $\Gamma_{j}$ centered at $\epsilon_{j}$; i.e., they satisfy the condition $\left|\epsilon_{j}-\epsilon_{m}\right| \lesssim \Gamma_{j}$. The miniband width is indicated with the gray shading area. Arrows of the same color depict transitions within one decay channel, connecting the state $\left|z_{j}\right\rangle$ to the states a Hamming distance $d$ away from it. Smaller values of $d$ correspond to bigger typical level spacings $\delta \epsilon_{j}^{d}$ [Eq. (87)] and fewer states in a miniband $\Omega_{d}$ [Eq. (98)] within the decay channel given by $d$.

$$
M_{j}^{(d)}=\sum_{m \neq j} \Delta\left(d-d_{j m}\right)
$$

As discussed in Appendix D, the elements of the set $\left\{M_{j}^{(d)}\right\}_{d=1}^{n}$ are sampled from the multinomial distribution with mean values

$$
\left\langle M_{j}^{(d)}\right\rangle=M p_{d}, \quad p_{d} \simeq 2^{-n}\left(\begin{array}{l}
n \\
d
\end{array}\right),
$$

where coefficient $p_{d}$ defined in Eq. (49) is the probability that a randomly chosen state is located a Hamming distance $d \neq 0$ from $\left|z_{j}\right\rangle$. The mean separation between the adjacent energies $\epsilon_{m}$ in the sum (84) equals

$$
\frac{W}{M_{j}^{(d)}} \sim \delta \epsilon \frac{2^{n}}{\left(\begin{array}{l}
n \\
d
\end{array}\right)}\left(M_{j}^{(d)} \geq 1\right),
$$

where $\delta \epsilon=W / M$ is the mean spacing between the marked state energies. A substantial contribution to the sum in Eq. (84) comes from the terms corresponding to the marked states whose energy levels $\epsilon_{j}$ lie within the width $\eta$ from the energy $\epsilon_{m}$; i.e., they satisfy the resonant condition $\left|\epsilon_{j}-\epsilon_{m}\right| \lesssim \eta$ as shown in Fig. 14.

The contribution to a sum from each resonance is $\sim 1 / \eta$ and the number of the resonances in a given decay channel is $\Omega_{d} \sim M_{j}^{(d)} \eta / W$ (cf. Fig. 14). It is shown in Appendix J that the dominant contribution to the typical values of $\Sigma_{j}^{\prime \prime}$ [Eq. (83)] comes from the values of $d$ that correspond to $\Omega_{d} \gg 1$. For them, the function $\delta\left(\epsilon_{j}-\epsilon_{m}, \eta\right)$ in Eq. (84) changes weakly between the adjacent values of $\epsilon_{m}$, and in the leading-order Born approximation we estimate the sum over $m$ in Eq. (84) by replacing it with an integral. Then, the spectral density can be estimated as

$$
\varrho_{\eta}^{j}(d) \simeq M_{j}^{(d)} p\left(\epsilon_{j}\right)
$$

where we require

$$
\delta \epsilon \ll \eta \ll W,
$$

and $p(\epsilon)$ is the PDF of the marked state energies $\epsilon$ with the width $W$ [see Eq. (47)].

We plug Eq. (88) into the expression (83), obtaining the following relation:

$$
\Sigma_{j}^{\prime \prime}=\pi p\left(\epsilon_{j}\right) \sum_{d=1}^{n} M_{j}^{(d)} V^{2}(d)[1-\cos 2 \phi(d)],
$$

where the sum is dominated by values of $d$ corresponding to large values $M_{j}^{(d)} \gg 1$ (see Appendix J). The steep exponential decrease with $d$ of the matrix element $V^{2}(d) \propto$ $1 /\left(\begin{array}{l}n \\ d\end{array}\right)$ [Eq. (42)] is canceled by equally steep growth with $d$ of the average number of states in the $d$ channel $\left\langle M_{j}^{(d)}\right\rangle \propto$ $\left(\begin{array}{l}n \\ d\end{array}\right)$ [Eq. (86)]. As a result, the binomial factors cancels out, and the average quantity $\left\langle M_{j}^{(d)}\right\rangle V^{2}(d)$ changes only by $\mathcal{O}\left(n^{-1}\right)$ when $d$ changes by 1 .

The term involving $\cos 2 \phi(d)$ above oscillates around 0 on the scale $d \sim 1$ [cf. Eq. (35)]. Therefore, the contributions to the sum from the terms $\propto\left\langle M_{j}^{(d)}\right\rangle \cos 2 \phi(d)$ average out. In what follows, we neglect the crossproduct of fluctuational and oscillatory parts $\left(M_{j}^{(d)}-\right.$ $\left.\left\langle M_{j}^{(d)}\right\rangle\right) \cos 2 \phi(d)$ and drop the second term in the rhs of Eq. (90) that contains $\cos 2 \phi(d)$.

Essentially, the above approximation corresponds to replacing the oscillatory part in the expression for the off-diagonal matrix elements $\mathcal{H}_{i j \neq i}=V\left(d_{i j}\right) \sqrt{2} \sin \phi\left(d_{i j}\right)$ [Eq. (41)] as follows:

$$
\mathcal{H}_{i j} \rightarrow V\left(d_{i j}\right) \beta_{i j}, \quad \beta_{i j}= \pm 1, \quad i<j,
$$

where $\beta_{i j}$ are instances of a dichotomous random variable that takes values of \pm 1 with probability $1 / 2$. This approximate model of the ensemble of $\mathcal{H}_{i j}$ is also used in the cavity method calculation in Sec. XI. 
Using the expression (47) for $p(\epsilon)$ and also Eqs. (56), (42), and (51), we obtain the relation between the PDFs of the random variables

$$
\begin{gathered}
\Sigma^{\prime \prime} \stackrel{d}{=} \Sigma_{*}^{\prime \prime} s_{M}, \quad \Sigma_{*}^{\prime \prime}=\pi \frac{V_{\mathrm{typ}}^{2}}{W / M}, \\
s_{M}=\frac{1}{M} \sum_{m=1}^{M} w_{m} .
\end{gathered}
$$

Here, $w_{m}$ are random variables independently sampled from the probability distribution $g_{\infty}(w)$ given in Eq. (60). The level widths $\Sigma_{j}^{\prime \prime}$ of individual marked states for $1 \leq j \leq M$ are samples of the random variable $\Sigma^{\prime \prime}$.

In Eq. (92), we introduce the characteristic value of the level width $\Sigma_{*}^{\prime \prime}$. This equation relates the PDF of $\Sigma^{\prime \prime}$ (or the decay rate $\Gamma=2 \Sigma^{\prime \prime}$ ) to that of $\epsilon$ and $M s_{M}$. We note that the resulting expression for the level width $\Sigma^{\prime \prime}$ of a marked state formally corresponds to that given by the FGR for the decay of the discrete level into the continuum [60]. The energies of the marked states $\epsilon_{m}$ into which a given marked state $\left|z_{j}\right\rangle$ decays form a miniband of the width $\Sigma_{j}^{\prime \prime}$. The decay occurs simultaneously in many channels corresponding to different Hamming distances between the initial marked state and the states of the miniband.

The heavy-tailed PDF of the random variable $s_{M}$ is studied in detail in Appendix I. Using the generalized central limit theorem (GCLT) for the sums of a large number of identical heavy-tailed random variables $[33,53]$, it can be represented in the form

$$
s_{M} \stackrel{d}{=} \sigma_{M} x+b_{M}
$$

where $x$ obeys a so-called Levy alpha-stable distribution $L_{1}^{1,1}(x)$ [33] defined in Appendix I, Eq. (I8), and shown in Fig. 15. The scaling factor and shift are

$$
\begin{gathered}
\sigma_{M}=\sqrt{\frac{\pi}{4 \log M}}, \\
b_{M} \simeq \sigma_{M}^{-1}-\frac{2}{\pi} \sigma_{M} \log \left(\sigma_{M}^{-1}\right)+\frac{2}{\pi}\left(1-\gamma_{\text {Euler }}\right) \sigma_{M},
\end{gathered}
$$

respectively $\left(\gamma_{\text {Euler }} \simeq 0.577\right.$ is the Euler constant). They display very weak logarithmic dependence on $M$ as compared with the main factor $\propto V_{\text {typ }}^{2} / \delta \epsilon$ in Eq. (92). The width of the PDF of $s_{M}$ is shrunk by a factor of $(\log M)^{1 / 2} \gg 1$, and the location of its maximum is increased by a factor of $(\log M)^{1 / 2} \gg 1$ compared to $L_{1}^{1,1}(x)$.

The PDF of $s_{M}$ has a polynomial tail. Therefore, decay rates of marked states $\Gamma_{j}=2 \Sigma_{j}^{\prime \prime}$ can take range values that are much bigger than their typical values $2 \Sigma_{*}^{\prime \prime}$ [Eq. (92)], up to $M$ times bigger in the sample of size $M$. These atypically large decay rates correspond to rare clusters of marked

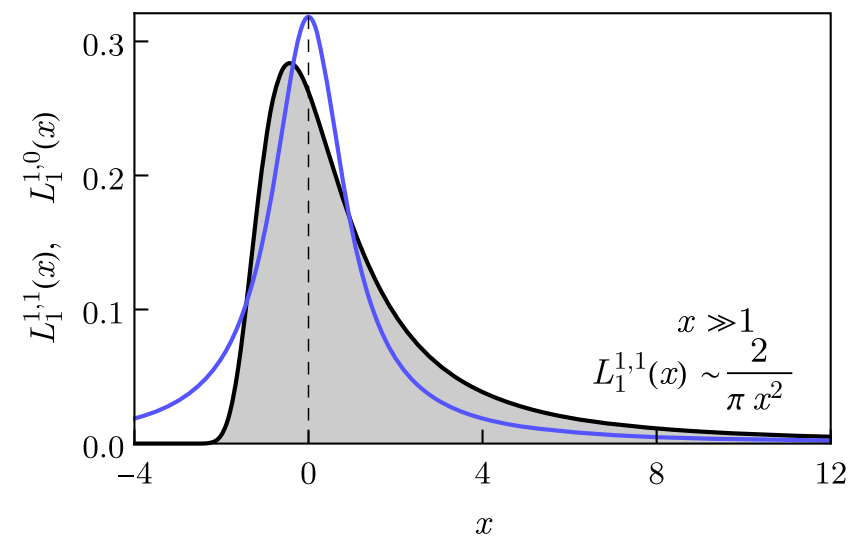

FIG. 15. The black solid line shows the plot of the Levy alphastable distribution $L_{\alpha}^{C, \beta}(x)$ [33] with tail index $\alpha=1$, asymmetry parameter $\beta=1$, and unit scale parameter $C=1$. The inset shows asymptotic behavior of the distribution at large positive $x$. At $-x \gg 1$, the function decays steeply as a double exponential, $\log L_{1}^{1,1}(x) \propto-\exp [-(\pi / 2) x]$. The blue line shows the Cauchy distribution $L_{1}^{1,0}(x)=\left\{1 /\left[\pi\left(1+x^{2}\right)\right]\right\}$. We follow here the definition introduced in Ref. [33] and used in subsequent papers on Levi matrices in the physics literature. In the mathematical literature $[61,62]$, a different definition is usually used, corresponding to $f\left(x ; \alpha, \beta, C^{1 / \alpha}, 0\right)=L_{\alpha}^{C, \beta}(x)$.

states that are located anomalously close to each other. When clusters are formed by $\mathcal{O}(1)$ states, the above picture of the decay fails.

\section{NUMBER OF STATES IN A MINIBAND WITHIN BORN APPROXIMATION}

Using the expression (92) for the miniband width, we can estimate the number of marked states $\Omega$ in a miniband corresponding to a given state $\left|z_{j}\right\rangle$. As before, we divide the states into groups of sizes $\Omega_{d}$, each corresponding to the transitions away from $\left|z_{j}\right\rangle$ with a fixed number of flipped bits $d$. The level width can be written in the form $\Sigma_{j}^{\prime \prime}=\sum_{d=1}^{M} \Sigma_{j, d}^{\prime \prime}$, where $\Sigma_{j, d}^{\prime \prime}$ is the partial level width due to the transitions with flipping $d$ bits. Then, using Eq. (90) and making use of the expression (86) for the average values of $M_{j}^{(d)}$, we obtain

$$
\Sigma_{j, d}^{\prime \prime} \simeq \pi \frac{V_{\mathrm{typ}}^{2}}{\delta \epsilon} \frac{1}{\sqrt{\pi n / 2}} \frac{M_{j}^{(d)}}{\left\langle M_{j}^{(d)}\right\rangle} .
$$

The quantity $M_{j}^{(d)} /\left\langle M_{j}^{(d)}\right\rangle \sim 1$ in Eq. (97), which results in the interesting phenomenon due to cancellation mentioned in the previous section: While the typical number of marked states in a decay channel varies very steeply with $d$, typical values of partial decay rates $\Sigma_{j, d}^{\prime \prime}$ in different channels do not. 
The estimate for the typical number of states in the miniband at the distance $d$ from $\left|z_{j}\right\rangle$ is $\Omega_{d} \sim \Sigma_{j, d}^{\prime \prime} / \delta \epsilon_{j}^{d}$ :

$$
\Omega_{d} \sim \Omega p_{d}, \quad \Omega \sim \frac{\Sigma_{j}^{\prime \prime}}{\delta \epsilon} \sim\left(\frac{V_{\mathrm{typ}}}{\delta \epsilon}\right)^{2},
$$

where $p_{d}=2^{-n}\left(\begin{array}{l}n \\ d\end{array}\right)$ and $\Omega$ is the total number of states in the miniband.

One can also write the partial decay rate as $\Gamma_{j}^{(d)} \sim$ $V(d) \Omega_{d}$, where the product $V(d) \Omega_{d}$ does not depend on $d$ (except from the prefactor). Of course, the analysis based on the decay rate does not apply for the transition to the channels with very few states. The condition $\Omega_{d} \simeq 1$ leads to $\Gamma_{j}^{(d)} \sim V(d)$ for $d=d_{\text {min }}^{\text {res }}$ corresponding to the typical Hamming distance from $\left|z_{j}\right\rangle$ to the nearest marked state in a miniband where the condition $V(d) \simeq \delta \epsilon_{j}^{d}$ is satisfied [see Eq. (J1) in Appendix J].

The above estimate gives the correct timescale $\sim 1 / V\left(d_{\min }^{\mathrm{res}}\right)$ over which the two states become hybridized. We note, however, that the total number of channels is $n-2 d_{\min }^{\text {res }}=\mathcal{O}(n)$. As all $\Gamma_{j}^{(d)}$ are nearly the same, each channel contributes a small fraction $\mathcal{O}(1 / n)$ to the total rate. Therefore, $V\left(d_{\min }^{\text {res }}\right) \sim \Gamma_{j} / n$, and marked state $\left|z_{j}\right\rangle$ decays into the large number of marked states within a miniband before it has a chance to hybridize with the nearest one at a distance $d_{\min }^{\text {res }}$. This property is markedly different from the situation at a finite dimension [15].

Using the scaling ansatz (75), we estimate the mean separation between the energies of marked states as

$$
\delta \epsilon=\frac{W}{M}=\lambda V_{\mathrm{typ}} M^{\gamma / 2-1} .
$$

Using Eqs. (92) and (98), we obtain the estimates for typical values of the decay rates and number of marked states in a miniband:

$$
\Gamma=2 \Sigma^{\prime \prime} \sim V_{\mathrm{typ}} M^{1-\gamma / 2}, \quad \Omega \sim M^{2-\gamma} .
$$

We immediately observe that in the range of $\gamma>2$ the number of marked states in a miniband vanishes. It corresponds to a localized phase, consistent with the fact that typical energy spacing $\delta$ becomes greater than the typical tunneling matrix element $V_{\text {typ }}$ connecting the states. The number of states in a miniband $\Omega$ cannot be greater than the total number of states $M$ in the IB. The expression above does not apply for $\gamma \leq 1$. This regime corresponds to the ergodic phase.

In the region $2>\gamma>1$, the separation between adjacent eigenvalues of $\mathcal{H}$ is of the same order as $\delta \epsilon$. The typical number of marked states in a miniband $\Omega$ corresponds to the typical number of nonergodic delocalized eigenstates of $\mathcal{H}$ that form the miniband:

$$
W \gg \Gamma \gg \delta \epsilon=\frac{W}{M} .
$$

The number of states in a miniband scales as a fractional power of $M$ less than one. This result is a hallmark of the nonergodic delocalized phase.

\section{CAVITY METHOD: SUMMARY OF THE PREVIOUS RESULTS}

The cavity method has been actively used to study Anderson localization in Levy matrices in the past several decades $[33-37,52,63]$ starting from the seminal work in Ref. [33]. In the present work, we use the cavity method to study the properties of minibands of delocalized nonergodic states that were previously discovered in the studies of the Rosenzweig-Porter [21,36] and regular random graph $[22,49]$ models. Initial studies suggest the existence of the mixed region with localized but nonergodic states [33]. However, recent numerical studies based on exact diagonalization using a very large number of samples establish that initially a large crossover region between localized and extended states collapses in the limit of increasing matrix sizes [35]. Multifractal properties of eigenstates in the localized phase and at criticality are studied in Ref. [37] using the strong disorder perturbation theory.

Numerical solutions of cavity equations to study localization transition in Levi matrices with power-law distributions $P\left(\mathcal{H}_{i j}^{2}\right) \propto 1 / \mathcal{H}_{i j}^{2(\alpha+1)}$ are obtained using the population dynamics algorithm [34] utilizing the approach developed in Ref. [63]. An alternative approach is based on the integral equation for the PDF of the diagonal elements of the resolvent $[33,52]$. It is obtained in the limit where the imaginary part of the self-energy is vanishingly small $[33,35,52]$ (with the limit of an infinite matrix size taken first). This approach allows one to derive analytically the global density of states [33,52] and the mobility edge $E^{*}(\alpha)$ which gives the $\alpha$ dependence of the energy $E^{*}$ separating extended and localized eigenvalues of $\mathcal{H}$ [35].

The cavity method proceeds as follows. First, we generate a random $M \times M$ matrix $\mathcal{H}_{i j}$ (41) from the ensemble described in Sec. VI. Then, we add a new row (and a symmetric column) of independent numbers identically distributed as those in the old matrix $\mathcal{H}_{i j}$. This process is done by generating a random energy $\epsilon_{0}$ from the distribution $(1 / W) p_{A}(\epsilon / W)$ and then generating a random bit string $z_{0}$, computing the array of Hamming distances $d_{j 0}$ between $z_{0}$ and $z_{j}$ and the corresponding matrix elements $\mathcal{H}_{j 0}=\mathcal{H}_{0 j}$ for integer $j \in[1, M]$. As a result, we obtain a new $(M+1) \times(M+1)$ matrix $\mathcal{H}^{+1}$, where +1 emphasizes that it has one more row and one more column than $\mathcal{H}$. We number elements of the new matrix by indices running over the range $[0, M]$, where the index 0 corresponds to the added marked state $\left|z_{0}\right\rangle$. The cavity equations have the form $[33,52]$ 


$$
\Sigma_{0}^{+1}(z)=\sum_{m=1}^{M} \mathcal{H}_{0 m}^{2} G_{m m}(z)
$$

where

$$
G_{m m}(z)=\left(z-\epsilon_{m}-\Sigma_{m}\right)^{-1}
$$

It does not involve the nondiagonal matrix elements of the Green's function $G_{m m^{\prime}}(z)$ when statistical average $\left\langle\mathcal{H}_{0 m}\right\rangle=0$, which is effectively our case as well [see Eq. (F1)].

The main assumption of the cavity method is that in the limit $M \rightarrow \infty$ the difference between the PDFs of $\Sigma_{0}^{+1}(z)$ and $\Sigma_{0}(z)$ disappears. This disappearance results in selfconsistent equations for the self-energy. Following Ref. [32], we add small imaginary parts to the diagonal matrix elements $\mathcal{H}_{m m}=\epsilon_{m}-i \eta$. It is a small "fictitious" quantity that is still assumed to be much bigger than the marked state energy spacing $\eta \gg W / M$. Results are not expected to depend on the value of $\eta$, provided its scaling with $M$ is chosen appropriately, as discussed below. We separate the real and imaginary parts of the self-energy, $\Sigma_{m}(z)=\Sigma_{m}^{\prime}(z)-i \Sigma_{m}^{\prime \prime}(z)$ [cf. Eq. (77)], obtaining

$$
\begin{aligned}
& \Sigma_{0}^{\prime} \stackrel{d}{=} \pi \sum_{m=1}^{M} \mathcal{H}_{0 m}^{2} \delta\left(\Sigma_{m}^{\prime \prime}+\eta, z-\epsilon_{m}-\Sigma_{m}^{\prime}\right), \\
& \Sigma_{0}^{\prime \prime} \stackrel{d}{=} \pi \sum_{m=1}^{M} \mathcal{H}_{0 m}^{2} \delta\left(z-\epsilon_{m}-\Sigma_{m}^{\prime}, \Sigma_{m}^{\prime \prime}+\eta\right),
\end{aligned}
$$

where the function $\delta(x, y) \equiv(1 / \pi)\left[y /\left(x^{2}+y^{2}\right)\right]$ is already introduced in Eq. (82).

The self-consistent Eqs. (102) were derived by AbouChacra, Anderson, and Thouless [32] for matrices on Bethe lattices and by Cizeau and Bouchaud for Levy matrices [33]. The solution of these equations was found only in the case when they can be linearized in $\Sigma_{m}^{\prime \prime}$ $[32,33,35]$, giving the location of mobility edge $E^{*}(\alpha)$ as a function of the power $\alpha$ in the tail of the PDF of the matrix elements $P\left(\mathcal{H}_{i j}^{2}\right) \propto 1 / \mathcal{H}_{i j}^{2(\alpha+1)}$. Here, we provide a full solution of the nonlinear equations.

We solve the self-consistent equations (102) under the assumption that pairs of variables $\left(\Sigma_{m}^{\prime}, \Sigma_{m}^{\prime \prime}\right)$ for each state $m \in[0, M]$ are taken from the same $\operatorname{PDF} \mathcal{P}\left(\Sigma^{\prime}, \Sigma^{\prime \prime} ; z\right)$ defined over the domain $x \in(-\infty, \infty), y \in[0, \infty)$. In what follows, for brevity, we omit the explicit dependence on the parameter $z$. Following Ref. [32], we introduce the characteristic function $\mathcal{F}\left(k_{1}, k_{2}\right)$ of the $\operatorname{PDF} \mathcal{P}\left(\Sigma^{\prime}, \Sigma^{\prime \prime}\right)$ :

$$
\mathcal{F}\left(k_{1}, k_{2}\right)=\int_{-\infty}^{\infty} d \Sigma^{\prime} \int_{0}^{\infty} d \Sigma^{\prime \prime} \mathcal{P}\left(\Sigma^{\prime}, \Sigma^{\prime \prime}\right) e^{i k_{1} \Sigma^{\prime}+i k_{2} \Sigma^{\prime \prime}},
$$

that satisfies the equation $\mathcal{F}_{\eta}\left(k_{1}, k_{2}\right)=\mathcal{G}_{\eta}^{M}\left(k_{1}, k_{2}\right)$, where

$$
\mathcal{G}\left(k_{1}, k_{2}\right)=\left\langle e^{i f k_{1} \delta\left(\eta+\Sigma^{\prime \prime}, z-\epsilon-\Sigma^{\prime}\right)+i f k_{2} \delta\left(z-\epsilon-\Sigma^{\prime}, \eta+\Sigma^{\prime \prime}\right)}\right\rangle .
$$

Here, $f=\mathcal{H}_{0 m}^{2}$ and the average is performed with the joint PDF $\mathcal{P}\left(\Sigma^{\prime}, \Sigma^{\prime \prime}\right)(1 / W) p_{A}(\epsilon / W) d f P(f)$. The above relation between $\mathcal{F}\left(k_{1}, k_{2}\right)$ and $\mathcal{G}\left(k_{1}, k_{2}\right)$ is actually an equation for the PDF $\mathcal{P}\left(\Sigma^{\prime}, \Sigma^{\prime \prime}\right)$, because both $\mathcal{G}$ and $\mathcal{F}$ depend on $\mathcal{P}$.

\section{SOLUTION OF CAVITY EQUATIONS IN NONERGODIC DELOCALIZED PHASE}

\section{A. Analysis of the imaginary part of self-energy}

We note that the exponent in the integrand of the above expression for $\mathcal{G}$ depends on $\Sigma^{\prime}$ and $\epsilon-z$ only via their combination $\Sigma^{\prime}+\epsilon-z$. In the nonergodic delocalized phase, the typical width of the PDF of $\Sigma^{\prime}$ is much more narrow than the width $W$ of $p(\epsilon)$ [Eq. (47)]. We also consider small values of $|z| \ll W$. Therefore, in the first approximation we neglect $\Sigma^{\prime}$ and $z$ compared to $\epsilon$. Then, $\mathcal{G}\left(k_{1}, k_{2}\right)$ depends only on the marginalized PDF

$$
\mathcal{P}\left(\Sigma^{\prime \prime}\right)=\int_{-\infty}^{\infty} d \Sigma^{\prime} \mathcal{P}\left(\Sigma^{\prime}, \Sigma^{\prime \prime}\right)
$$

Once this $\operatorname{PDF}$ is obtained, the $\operatorname{PDF} \mathcal{P}\left(\Sigma^{\prime}, \Sigma^{\prime \prime}\right)$ can be analyzed from its characteristic function $\mathcal{F}\left(0, k_{2}\right)$. Inverting it, we obtain the self-consistent equation for $\mathcal{P}\left(\Sigma^{\prime \prime}\right)$ in the limit $M \rightarrow \infty$ :

$$
\begin{aligned}
\mathcal{P}\left(\Sigma^{\prime \prime}\right) & =\frac{1}{2 \pi} \int_{-\infty}^{\infty} d k e^{M \theta(k)-i k \Sigma^{\prime \prime}}, \\
\theta(k) & =\int_{0}^{\infty} d f d \Sigma^{\prime \prime} d h P(f) \mathcal{P}\left(\Sigma^{\prime \prime}\right) p_{\eta+\Sigma^{\prime \prime}}(h)\left(e^{i k f h}-1\right) .
\end{aligned}
$$

Here, $\theta(k)=1-\mathcal{G}_{\eta}(0, k)$ and the domain of integration for all variables is $[0, \infty)$. The function $p_{\eta+Y}(h)$ above is a conditional PDF of a random variable

$$
h=\delta(\epsilon, \eta+Y)
$$

with $Y$ fixed and $\delta(x, y)$ given in Eq. (82). The explicit form of the PDF $p_{\eta+Y}(h)$ is obtained in Appendix K, Eqs. (K6) and (K8).

To achieve further progress, we use the approximation (91) and drop oscillatory factors in the off-diagonal matrix elements $\mathcal{H}_{0 m}$. Then, we have for the PDF $P(f)=$ $g_{\infty}\left(f / V_{\text {typ }}^{2}\right) / V_{\text {typ }}^{2}$ [Eq. (60)], and in what follows we use the rescaled variable $w=f / V_{\text {typ }}^{2}$ for the squared matrix elements, in accordance with Eq. (56). Instead of the variable $h$ in Eq. (104), we use the rescaled variable

$$
y=\sqrt{h\left(\eta+\Sigma^{\prime \prime}\right)},
$$

that obeys the distribution 


$$
\mathfrak{p}_{\eta+\Sigma^{\prime \prime}}(y)=\frac{2\left(\eta+\Sigma^{\prime \prime}\right)}{W} \frac{1}{y^{2} \sqrt{1-y^{2}}}
$$

[see details in Appendix K, Eq. (K19)]. Then, $\theta(k)$ takes the form

$$
\theta(k)=\int_{0}^{\infty} d \Sigma^{\prime \prime} \mathcal{P}\left(\Sigma^{\prime \prime}\right) \phi_{\Sigma^{\prime \prime}+\eta}\left(\frac{k V_{\mathrm{typ}}^{2}}{\Sigma^{\prime \prime}+\eta}\right)
$$

Here, $\phi_{Y}(u)$ is a characteristic function

$$
\phi_{Y}(u)=\int_{0}^{\infty} d x g_{Y}(x)\left(e^{i u x}-1\right)
$$

of the PDF $g_{Y}(x)$ of the random variable $x=w y^{2}$, where $w$ obeys $g_{\infty}(w)$ and $y$ obeys $\mathfrak{p}_{Y}(y)$ [Eq. (106)]. A detailed study of $g_{Y}(x)$ is given in Appendix L. The PDF $g_{Y}(x)$ depends on $Y$ via the ratio $Y / W$, and its plot is shown in Fig. 16. It goes over into $g_{\infty}(y)$ for $Y \rightarrow \infty$.

We now make a key observation: In the limit of large $x \gg 1$ and for $W \gg Y$, the following relations hold for the PDF $\phi_{Y}(u)$ and its characteristic function [see the corresponding Eqs. (L26) and (L10) in Appendix L]:

$$
g_{Y}(x) \simeq \frac{\pi Y}{W} g_{\infty}(x), \quad \phi_{Y}(u) \simeq \frac{\pi Y}{W} \phi_{\infty}(u)
$$

The reason for this result can be explained as follows. For large deviations of $x=w Y^{2} /\left(\epsilon^{2}+Y^{2}\right)$, the conditional PDF $p(\epsilon \mid x)$ of the marked state energy $\epsilon$ is narrowly peaked in the range of values $|\epsilon| \sim Y$. In contrast, typical energy values are much bigger: $\epsilon \sim W$. This narrowing of the

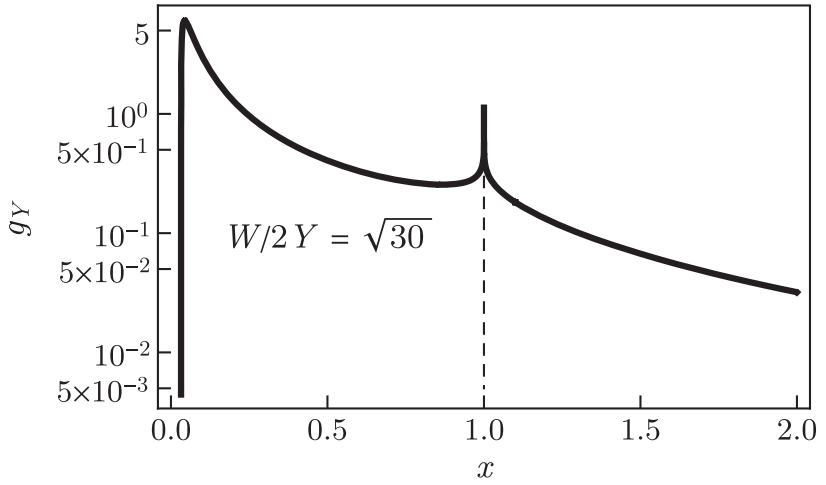

FIG. 16. Plot of the PDF $g_{Y}(x)$ of the random variable $x=\left\{\left(w Y^{2}\right) /\left[(z-\epsilon)^{2}+Y^{2}\right]\right\}$, where random variables $\epsilon$ and $w$ obey distributions $W^{-1} p_{A}(\epsilon / W)$ and $g_{\infty}(w)$, respectively, and $W /(2 Y)=\sqrt{30}$. A detailed discussion of $g_{Y}(x)$ is given in Appendix L [see Eq. (L7)]. Its maximum is located at $x \sim(Y / W)^{2}$. The singularity at $x=1$ corresponds to $\epsilon=z$. For large values of $x \gg 1$, the conditional PDF of $\left\{Y^{2} /\left[(z-\epsilon)^{2}+\right.\right.$ $\left.\left.X^{2}\right]\right\}$ is narrowly peaked around its mean value $\pi Y / W$ with $|\epsilon-z| \sim Y$, giving rise to the relation in Eq. (109). conditional PDF $p(\epsilon \mid x)$ gives rise to a small factor $\pi Y / W$ in the rhs of Eq. (109).

We observe that $\lim _{k \rightarrow \infty} \theta(k)=0$ and for $M \rightarrow \infty$ the integral in Eq. (104) is dominated by $|k| \ll 1$. We make an assumption (whose validity becomes obvious below) that for small enough $k$ the integral in Eq. (107) is dominated by values of $\Sigma^{\prime \prime}$ such that $k V_{\text {typ }}^{2} /\left(\Sigma^{\prime \prime}+\eta\right) \ll 1$. Therefore, we use in Eq. (107) the approximate expression for the characteristic function $\phi_{\Sigma^{\prime \prime}+\eta}$ given by Eq. (109). We rescale $\Sigma^{\prime \prime}$ with the typical value of the imaginary part of the self-energy of marked states $\Sigma_{*}^{\prime \prime}$ [Eq. (92)] obtained in the FGR-based calculation in Sec. VIII. Making a change of variables

$$
\Sigma^{\prime \prime}=\Sigma_{*}^{\prime \prime} s, \quad \mathcal{P}\left(\Sigma^{\prime \prime}\right)=\frac{1}{\Sigma_{*}^{\prime \prime}} \rho(s),
$$

we rewrite the self-consistent equation (104) in the limit $x \gg 1, W \gg Y$ for the rescaled $\operatorname{PDF} \rho(s)$ in the following form:

$$
\begin{gathered}
\rho(s)=\frac{1}{2 \pi} \int_{-\infty}^{\infty} d u e^{-i u s+\Phi(u, \Omega)}, \\
\Phi(u, \Omega)=\int_{-\infty}^{\infty} d \nu \rho\left(\nu-\beta_{\eta}\right) \Omega \nu \phi_{\infty}\left(\frac{q}{\Omega \nu}\right), \\
\beta_{\eta}=\frac{\eta}{\Sigma_{*}^{\prime \prime}},
\end{gathered}
$$

and

$$
\Omega=\frac{\pi \Sigma_{*}^{\prime \prime}}{\delta \epsilon}=\left(\frac{\pi V_{\mathrm{typ}}}{W / M}\right)^{2} .
$$

$\Sigma_{*}^{\prime \prime}$ and $V_{\text {typ }}$ are defined in Eqs. (92) and (51), respectively. We observe that $\Omega$ corresponds to the typical number of marked states in the miniband that we estimate in Sec. VIII using the Born approximation.

Assuming $\Omega \gg 1$ (delocalized phase), we expand $\Omega \nu \phi_{\infty}[q /(\Omega \nu)]$ in inverse powers of $\log \Omega$ using the asymptotic form of the characteristic function $\phi_{\infty}(u)$ at the small argument studied in Appendix H, Eqs. (H13) and (H14). Truncating the expansion at terms $\sim(\log M)^{-1 / 2}$, we get

$\Omega \operatorname{Re} \phi_{\infty}\left(\frac{q}{\Omega}\right) \simeq-\frac{\pi|q|}{2 \sqrt{\log \Omega}}$,

$\Omega \operatorname{Im} \phi_{\infty}\left(\frac{q}{\Omega}\right) \simeq 2 q\left(\frac{\log \Omega}{\pi}\right)^{1 / 2}+q \frac{1-\mathcal{C}-\log |q|}{(\pi \log \Omega)^{1 / 2}}$,

where $\mathcal{C} \approx 0.577$ is the Euler constant. It is clear from comparing individual terms in Eq. (114) with the exponential in 
Eq. (111) that $q=\mathcal{O}(\sqrt{\log \Omega})$. This result justifies the order of truncation [see details in Appendix I, Eq. (I5)].

We make a change of variables in the integral in Eq. (111), $q=2 \sqrt{\log \Omega / \pi} t$, and obtain

$$
\rho(s)=\sigma_{\Omega}^{-1} L_{1}^{1,1}\left[\left(s-\mu_{\Omega}\right) / \sigma_{\Omega}\right],
$$

where the quantity $\mu_{\Omega}$ satisfies the equation

$$
\mu_{\Omega}=b_{\Omega}+\frac{2 \sigma_{\Omega}}{\pi} \int_{-\infty}^{\infty} d s \rho(s) \log \left|s+\beta_{\eta}\right|
$$

Above $L_{1}^{1,1}(x)$ is the Levy distribution [33] defined in Appendix I, Eq. (I8), and shown in Fig. 15. Coefficients $\sigma_{\Omega}=\sqrt{\pi /(4 \log \Omega)}$ and $b_{\Omega} \simeq 1 / \sigma_{\Omega}$ are given in Eqs. (95) and (96), where the parameter $M$ needs to be replaced by $\Omega$.

We plug the above expression for $\rho(s)$ into Eq. (116) and express $\mu_{\Omega}$ in terms of a new variable $x$ :

$$
\mu_{\Omega} \equiv b_{\Omega}-\frac{2 \sigma_{\Omega}}{\pi} \log \sigma_{\Omega}^{-1}+\sigma_{\Omega} x .
$$

Then, this variable satisfies the following equation:

$$
x=\frac{2}{\pi} \int_{-\infty}^{\infty} d s L_{1}^{1,1}(s) \log \left|s+x+\zeta_{\Omega}\right|,
$$

that involves a scale-free Levy distribution and a single parameter $\zeta_{\Omega}$ :

$$
\zeta_{\Omega}=\frac{b_{\Omega}}{\sigma_{\Omega}}-\frac{2}{\pi} \log \left(\frac{1}{\sigma_{\Omega}}\right)+\frac{1}{\sigma_{\Omega}} \frac{\eta}{\Sigma_{*}^{\prime \prime}},
$$

where we use an explicit form of $\beta_{\eta}$ [Eq. (112)]. We note that the self-consistent equation for the function $\rho(s)$ is now reduced to the simple transcendental equation (118).

Using the explicit form of $\sigma_{\Omega}$ and $b_{\Omega}$ [Eqs. (95) and (96)], one can see that $\zeta_{\Omega}$ is large compared to unity in the delocalized phase: $\zeta_{\Omega} \simeq \sigma_{\Omega}^{-2} \sim \log \Omega \gg 1$. With this property, the equation for $x$ (118) can be solved by iteration using the asymptotic expansion of the Levy distribution at large arguments: $L_{1}^{1,1}(\nu) \simeq(2 / \pi) \nu^{-2}(\nu \gg 1)$. To leading order,

$$
x \simeq \frac{2}{\pi} \log \zeta_{\Omega}+\mathcal{O}\left(\frac{\log \zeta_{\Omega}}{\zeta_{\Omega}}\right) .
$$

Then, using Eq. (117), the expression for $\mu_{\Omega}$ is

$$
\mu_{\Omega} \simeq \frac{1}{\sigma_{\Omega}}+\frac{2 \sigma_{\Omega}}{\pi} \log \left(1+\frac{\eta \sigma_{\Omega}}{\Sigma_{*}^{\prime \prime}}\right)+\frac{2 \sigma_{\Omega}\left(1-\gamma_{\text {Euler }}\right)}{\pi},
$$

where we neglect terms $\sim \sigma_{\Omega}^{3} \log \Omega$ that are much smaller than the width $\sigma_{\Omega}$ of the distribution $\rho(s)=$ $\sigma_{\Omega}^{-1} L_{1}^{1,1}\left[\left(s-\mu_{\Omega}\right) / \sigma_{\Omega}\right]$.

We note that the dependence of $\mu_{\Omega}$ [Eq. (121)] on the initial (fictitious) level broadening $\eta$ disappears when the latter is chosen to be much smaller than the miniband width [22,36,49] $W / M \ll \eta \ll \Sigma_{*}^{\prime \prime} \sigma_{\Omega}$. Using Eqs. (92) and (75), the scaling behavior of $\eta$ with $M$ in the nonergodic delocalized regime must satisfy the condition

$$
\eta=M^{\kappa}, \quad|\kappa|<1-\frac{\gamma}{2}, \quad \gamma \in(1,2) .
$$

Finally, the expression for the distribution function of the imaginary part of the self-energy has the form

$$
\begin{aligned}
& \mathcal{P}\left(\Sigma^{\prime \prime}\right)=\frac{1}{C} L_{1}^{1,1}\left(\frac{\Sigma^{\prime \prime}-\Sigma_{\text {typ }}^{\prime \prime}}{C}\right), \\
& \Sigma_{\text {typ }}^{\prime \prime}=\mu_{\Omega} \Sigma_{*}^{\prime \prime}, \quad C=\sigma_{\Omega} \Sigma_{*}^{\prime \prime} .
\end{aligned}
$$

Here, $\Sigma_{\text {typ }}^{\prime \prime}$ is a shift of the distribution and $C$ its scale parameter (characteristic width). Also,

$$
\begin{gathered}
\mu_{\Omega} \simeq \frac{1}{\sigma_{\Omega}}+\frac{2 \sigma_{\Omega}\left(1-\gamma_{\text {Euler }}\right)}{\pi}, \\
\sigma_{\Omega}=\sqrt{\frac{\pi}{4 \log \Omega}} .
\end{gathered}
$$

Using the scaling ansatz (75) for the width $W$ of the IB in terms of $M$, the typical number of states in a miniband (number of resonances) equals

$$
\Omega=\left(\frac{\pi}{\lambda}\right)^{2} M^{2-\gamma} .
$$

Using the same scaling ansatz (75) and the expressions for $\sigma_{\Omega}$ [Eq. (95)] and $\mu_{\Omega}$ [Eq. (125)], we obtain

$$
\begin{gathered}
\Sigma_{\mathrm{typ}}^{\prime \prime} \simeq \frac{2 \pi^{1 / 2}}{\lambda} V_{\mathrm{typ}} M^{1-\gamma / 2}(\log \Omega)^{1 / 2}, \\
C \simeq \frac{\pi^{3 / 2}}{2 \lambda} V_{\mathrm{typ}} M^{1-\gamma / 2}(\log \Omega)^{-1 / 2}
\end{gathered}
$$

Here, $V_{\text {typ }} \sim n^{1 / 2} 2^{-n / 2} e^{-n /\left(4 B_{\perp}^{2}\right)}$ is given in Eq. (51). The shift $\Sigma_{\text {typ }}^{\prime \prime}$ corresponds to the typical value of $\Sigma^{\prime \prime}$. One can see from the above that it is $\log \Omega \sim \log M \gg 1$ times bigger than the distribution width. We note in passing that the distribution of $\Sigma^{\prime \prime}$ determines that of the miniband width $\Gamma=2 \Sigma^{\prime \prime}$ [Eq. (100)]. 


\section{Comparison between the cavity method and leading-order Born approximation}

It is instructive to compare the above distribution of $\Sigma^{\prime \prime}$ obtained using the cavity method with that obtained within the Born approximation (92)-(96). In both cases, the distribution of $\Sigma^{\prime \prime}$ is given by the appropriately rescaled and shifted Levy alpha-stable distribution $L_{1}^{1,1}(x)$. In both cases, the scale parameter $C$ (characteristic width) of the distribution has the form $C=\sigma_{S} \Sigma_{*}^{\prime \prime}$ with $\sigma_{S}=\sqrt{\pi /(4 \log S)}$. In the case of the Born approximation $S=M$, corresponding to the total number of marked states, and in the case of cavity method $S=\Omega \ll M$, corresponding to the (much smaller) number of states in the miniband. Using Eq. (127), we estimate

$$
\frac{\sigma_{M}}{\sigma_{\Omega}}=\sqrt{2-\gamma}<1 \quad\left(W=\lambda M^{\gamma / 2}\right) .
$$

Therefore, the Born approximation underestimates the width of the distribution of $\Sigma^{\prime \prime}$. The ratio (130) is especially pronounced near the localization transition $\gamma=2$. The value of $\sigma_{\Omega}^{-1}$ shrinks to zero at the transition, while that of $\sigma_{M}$ does not depend on the closeness to the transition point.

We note, however, that factors $\sigma_{\Omega}$ and $\sigma_{M}$ depend on $M$ only logarithmically. At the same time, the leadingorder (power-law) dependence of the rescaling coefficient on $M$ is given by the factor $\Sigma_{*}^{\prime \prime} \propto M^{1-\gamma / 2}$ and is identical in the cavity method and the Born-approximation-based expressions.

The situation is similar with the shift parameter $\Sigma_{\text {typ }}^{\prime \prime}$ in the Levy distribution of $\Sigma^{\prime \prime}$ corresponding its typical value $\Sigma_{\text {typ }}^{\prime \prime} \simeq \Sigma_{*}^{\prime \prime} / \sigma_{S}$ with $S=M$ (Born approximation) and $S=\Omega$ (cavity method). The leading-order dependence of the shift on $M$ is the same in both cases and is given by $\Sigma_{*}^{\prime \prime}$. In both cases, the shift is greater than the rescaling coefficient by a factor of $\sim \log M$. However, the Born approximation overestimates the shift by a factor of $(2-\gamma)^{-1 / 2}$.

\section{Comparison between the cavity method and self-consistent Born approximation}

The leading-order Born approximation recovers the typical shift $\Sigma_{\text {typ }}^{\prime \prime}$ and the scale parameter $C$ of the distribution of $\Sigma^{\prime \prime}$ with exponential accuracy in $\log M$. However, it gives an incorrect dependence of the prefactor on $\log M$ in these coefficients. The main approximation in Sec. VIII A is to assume that the sum in the expression for the spectral density $\rho_{\eta}^{j}(d)$ [Eq. (84)] can be replaced by an integral. We revisit the decay rate equation (81) using the statistical ensemble (91):

$$
\Sigma^{\prime \prime}=\frac{V_{\mathrm{typ}}^{2}}{\eta} \sum_{m=1}^{M} x_{m}, \quad x_{m}=\frac{w_{m} \eta^{2}}{\epsilon_{m}^{2}+\eta^{2}} .
$$

Here in the lhs, we omit the subscript in $\Sigma_{j}^{\prime \prime}$ and make the rescaling $V\left(d_{j m}\right)^{2}=V_{\text {typ }}^{2} w_{m}$. Random variables $x_{m}$ are sampled from the distribution $g_{\eta}(x)$ given in Eq. (109) and plotted in Fig. 16. Using the GCLT for the sum in Eq. (131), one can obtain the PDF of $\Sigma^{\prime \prime}$. The details are given in Appendix L, and here we provide the result:

$$
\Sigma^{\prime \prime} \stackrel{d}{=} \tilde{\Sigma}_{\text {typ }}^{\prime \prime}+x C, \quad \tilde{\Sigma}_{\text {typ }}^{\prime \prime}=b_{\Omega_{\eta}} \Sigma_{*}^{\prime \prime}, \quad C=\sigma_{\Omega_{\eta}} \Sigma_{*}^{\prime \prime} .
$$

Here, $x$ is a random variable that obeys Levy distribution $L_{1}^{1,1}(x)$, coefficient $\sigma_{\Omega}$ is given in Eq. (126), and $b_{\Omega}$ is given in Eq. (96), where one should replace $M$ with the number of marked states in a miniband of width $\eta$ :

$$
\Omega_{\eta}=\frac{\pi \eta}{\delta \epsilon} .
$$

Unlike the discussion in the cavity method, the statistics of $\Sigma^{\prime \prime}$ explicitly depends on $\eta$. We make a self-consistent assumption and set $\eta$ equal to the characteristic width of the miniband:

$$
\eta=\Sigma_{*}^{\prime \prime} \Rightarrow \Omega_{\eta}=\frac{\pi \Sigma_{*}^{\prime \prime}}{\delta \epsilon} .
$$

We conclude that the typical number of states in a miniband $\Omega_{\eta}=\Omega$ given by the self-consistent Born approximation is the same as that given by the cavity method [Eqs. (113)]. Therefore, using Eq. (132), one can see that the width $C$ of the distribution of $\Sigma^{\prime \prime}$ is also the same in both methods. The difference between the typical values of $\Sigma^{\prime \prime}$ in the two methods is

$$
\Sigma_{\text {typ }}^{\prime \prime}-\tilde{\Sigma}_{\text {typ }}^{\prime \prime}=\frac{2}{\pi} C \log \sigma_{\Omega}^{-1} \ll \Sigma_{\text {typ }}^{\prime \prime} .
$$

This error is much smaller than in the case discussed in Sec. XI A 1 [cf. Eq. (130)], where the self-consistent condition is not used. However, it exceeds the distribution width $C$ for sufficiently large $M \gg 1$, because in the nonergodic delocalized phase $\log \sigma_{\Omega}^{-1} \sim \log \log M$.

\section{B. Real part of self-energy}

In this section, we find the marginalized probability distribution of real parts of the self-energy:

$$
\mathcal{P}\left(\Sigma^{\prime}\right)=\int_{0}^{\infty} d \Sigma^{\prime \prime} \mathcal{P}\left(\Sigma^{\prime}, \Sigma^{\prime \prime}\right)
$$

We consider the first equation in Eq. (102). Following the arguments provided in Sec. XI A, we neglect the terms $z-\Sigma_{m}^{\prime}$ in the rhs of the equation and drop the oscillatory factors in $\mathcal{H}_{0 m}$ using the probability distribution $P(f)=$ $g_{\infty}\left(f / V_{\text {typ }}^{2}\right) / V_{\text {typ }}^{2}$ [Eq. (60)] instead. Then, Eq. (102) takes the form 


$$
\Sigma^{\prime} \stackrel{d}{=} \sum_{m=1}^{M} r_{m}
$$

Here, $r_{m}$ are instances of a random variable $R$ such that

$$
r=f \frac{\epsilon}{\epsilon^{2}+\left(\Sigma^{\prime \prime}\right)^{2}},
$$

where $\epsilon, f$, and $\Sigma^{\prime \prime}$ are random variables independently sampled from the distributions $p(\epsilon), P(f)$, and $\mathcal{P}\left(\Sigma^{\prime \prime}\right)$, respectively. Using the GCLT, in the asymptotic limit of $M \rightarrow \infty$ the sum in Eq. (136) is determined by the tail of the probability distribution of $r$ at $|r| \rightarrow \infty$. This analysis is very similar to the one already discussed in Secs. VIII and XI A and in Appendix I. Here, we omit details of the calculations and simply provide the result. The tail of the PDF of $r$ in the limit $|r| \rightarrow \infty$ has the form

$$
\rho=\frac{r}{2 \Sigma_{*}^{\prime \prime} /(\pi M)}, \quad \operatorname{PDF}(\rho) \simeq \frac{1}{\rho^{2}} \sqrt{\frac{\log (\rho)}{\pi}}
$$

$(\rho \gg 1)$. The distribution function $\mathcal{P}\left(\Sigma^{\prime}\right)$ of the sum in Eq. (136) is the Cauchy distribution

$$
\mathcal{P}\left(\Sigma^{\prime}\right)=\frac{1}{\pi} \frac{\Sigma_{\mathrm{typ}}^{\prime}}{\pi\left(\Sigma_{\mathrm{typ}}^{\prime}\right)^{2}+\left(\Sigma^{\prime}\right)^{2}}, \quad \Sigma_{\mathrm{typ}}^{\prime}=\frac{\Sigma_{*}^{\prime \prime}}{\sigma_{M}} .
$$

Here, the expression for $\sigma_{M} \sim 1 / \sqrt{\log M}$ is given in Eq. (95). The Cauchy distribution has a form very similar to the stable distribution $L_{1}^{1,1}(x)$ that describes the fluctuations of the $\Sigma^{\prime \prime}$ [Eq. (123)] up to the shift and rescaling coefficients. Both distributions are displayed in Fig. 15. The tail of the Cauchy distribution differs from that of $L_{1}^{1,1}(x)$ by a factor of 2 . Unlike that of $\Sigma^{\prime \prime}$, the distribution of $\Sigma^{\prime}$ is symmetric for impurity states with energies near the center of the band. The typical value of $\Sigma^{\prime}$ is greater than that of $\Sigma^{\prime \prime}$ by a constant factor

$$
\frac{\Sigma_{\text {typ }}^{\prime}}{\Sigma_{\text {typ }}^{\prime \prime}}=\frac{1}{\sqrt{2-\gamma}} \quad\left(W=\lambda M^{\gamma / 2}\right) .
$$

The width of the distribution of $\Sigma^{\prime}$ is the same as its typical value, while the width $C$ of the distribution of $\Sigma^{\prime \prime}$ is smaller by a factor of $\sim 1 / \log M$ [cf. Eqs. (128) and (129)]. These relations between the distributions of $\Sigma^{\prime}$ and $\Sigma^{\prime \prime}$ have implications for the complexity of the population transfer as discussed below. We also note that the real and imaginary parts of the self-energy of a given marked state are correlated with each other, because, according to Eqs. (102a) and (102b), the values of $\Sigma_{j}^{\prime}$ and $\Sigma_{j}^{\prime \prime}$ depend on the same set of parameters $\left(\mathcal{H}_{j m}, \epsilon_{m}\right.$, etc). In this work, we do not study their correlations.

\section{Dynamic correlations}

For states close to the center of the band of marked states, the typical value of the miniband width can be connected to the average of the dynamical correlator, with the delta function regularized by a finite scale $\eta, \Sigma_{\text {typ }}^{\prime \prime} \gg \eta \gg \delta \epsilon$, $\delta(x) \rightarrow \delta_{\eta}(x) \equiv(1 / \pi)\left[\eta /\left(x^{2}+\eta^{2}\right)\right]$,

$$
\frac{1}{\Sigma_{\text {typ }}^{\prime \prime}+\eta}=\frac{1}{\pi} \int_{-\infty}^{\infty} d \omega \frac{\eta}{\eta^{2}+\omega^{2}} p(\omega),
$$

which can be inverted to obtain

$$
p(\omega) \approx\left[\begin{array}{ll}
\frac{1}{\Sigma_{\text {typ }}^{\prime \prime}}, & \omega \leq \omega_{\mathrm{Th}}, \\
\frac{1}{\Sigma_{\text {typ }}^{\prime \prime}}\left(\frac{\omega_{\mathrm{Th}}}{|\omega|}\right)^{2}, & \omega>\omega_{\mathrm{Th}},
\end{array}\right.
$$

where we introduce the Thouless energy

$$
\omega_{\mathrm{Th}}=\frac{1}{2} \pi \Sigma_{\mathrm{typ}}^{\prime \prime} .
$$

The typical value of the miniband width is obtained in Eq. (128). From the comparison of the respective Fig. 12, we conclude that the scaling of the typical population transfer time $1 / \omega_{\mathrm{Th}}$ and the scaling of the value of the dynamical correlator $K(\omega)$ are consistent in numerical and analytical calculations, subject only to a small correction in the scaling exponent $\varepsilon=0.05$.

\section{COMPLEXITY OF THE POPULATION TRANSFER PROTOCOL}

After the system is prepared at a given marked state $\left|z_{j}\right\rangle$ at $t=0$, the probability for the population to be transferred to other marked states is $1-\psi^{2}\left(z_{j}, t\right)$. At the initial stage, the survival probability decays exponentially [Eq. (79)] with the mean decay time $1 / \Gamma_{j}=1 /\left(2 \Sigma_{j}^{\prime \prime}\right)$.

The initial marked state decays into the eigenstates $\left|\psi_{\beta}\right\rangle$ of the IB Hamiltonian $\mathcal{H}$ with typical energies $E_{\beta}$ inside the narrow interval corresponding to the miniband associated with $\left|z_{j}\right\rangle$. It has a width $\Sigma_{j}^{\prime \prime}$ and is centered around $\mathcal{H}_{j j}=\epsilon_{j}$. Typical classical energies $\epsilon$ of the bit strings measured at the end of PT protocol obey the probability distribution $\mathcal{P}\left(\epsilon-\epsilon_{j}-\Sigma_{j}^{\prime}\right)$ with $\mathcal{P}$ given in Eq. (139). The success of the PT protocol is to find a bit string distinct from $z_{j}$ at a time $t$ with energy inside that window $\Delta \mathcal{E}_{\mathrm{cl}}$ around $\epsilon_{j}$. The expected time to succeed in the PT equals

$$
t_{\mathrm{PT}}^{j}=\frac{1}{2 \Sigma_{j}^{\prime \prime} p_{\Delta \mathcal{E}}}, \quad p_{\Delta \mathcal{E}}=\int_{0}^{\Delta \mathcal{E}_{\mathrm{cl}}} \mathcal{P}\left(\epsilon-\Sigma_{j}^{\prime}-\frac{\Delta \mathcal{E}_{\mathrm{cl}}}{2}\right) d \epsilon .
$$

Here, $p_{\Delta \mathcal{E}}$ is the probability of detecting a bit string inside the target window $\Delta \mathcal{E}_{\mathrm{cl}}$ under the condition that the initial state has decayed. Let us assume that the PT window is as wide as the typical miniband width, $\Delta \mathcal{E}_{\mathrm{cl}}=\Sigma_{\text {typ. }}^{\prime \prime}$. In this 
case, $p_{\mathrm{mb}}$ differs from 1 only by a constant factor that does not depend on $M$ [cf. Eq. (140)]. Therefore, we detect the bit string inside the PT window with a finite probability as long as we wait long enough for the transition away from the initial marked state to occur. Because the initial state $\left|z_{j}\right\rangle$ is picked at random, we can estimate the typical time to success of PT $t_{\mathrm{PT}} \sim 1 / \Sigma_{\text {typ }}^{\prime \prime}$ corresponding to the inverse typical width of the miniband. All of the states in a miniband are populated at (roughly) the same time $t_{\mathrm{PT}}$, because the transition rate to a subset of states on a distance $d$ away from $\left|z_{j}\right\rangle$ depends on $d$ very weakly [see Eq. (97) and the related discussion in Sec. IX].

From a computational perspective, it is of interest to characterize the PT by the relation between the typical success time of PT $t_{\mathrm{PT}}$ and the number of states $\Omega$ over which the population is spread during the PT:

$$
t_{\mathrm{PT}} \sim \frac{1}{V_{\mathrm{typ}} \sqrt{\Omega \log \Omega}} \sim\left(\frac{2^{n}}{n \Omega \log \Omega}\right)^{1 / 2} e^{2 \theta n}
$$

where we set $\Delta \mathcal{E}_{\mathrm{cl}} \sim \Sigma_{*}^{\prime \prime}$ (see the discussion above). We note that the time $t_{G}$ for the Grover algorithm for an unstructured quantum search to find $\Omega$ items in a database of the size $2^{n}$ is $t_{G} \sim\left(2^{n} / \Omega\right)^{1 / 2}$. PT time $t_{\mathrm{PT}}$ scales worse than Grover time $t_{G}$ by an additional exponential factor $e^{2 \theta n} \simeq e^{\left[n /\left(2 B_{\perp}^{2}\right)\right]}$ [Eq. (46)]. The scaling exponent $2 \theta$ can be made arbitrarily small at large transverse fields $1 \ll B_{\perp}=\mathcal{O}\left(n^{0}\right)$.

One can expect that the distributions of eigenvalues and eigenvectors inside the miniband are very similar to those in the ergodic case, albeit with the appropriately rescaled effective dimension $\Omega$ of the Hilbert space [21]. For example, the energy spectrum of the minibands in the nonergodic delocalized phase of the RP model corresponds to the Gaussian orthogonal ensemble. There, according to the semicircle law [64], the typical spectral width of the miniband (approximately $1 / t_{\mathrm{PT}}$ ) is proportional to the square root of the number of states $\Omega$ in it. Therefore, the Grover scaling (144) for the PT is consistent with the semicircle law in the Gaussian random matrix models that allow for a nonergodic delocalized phase such as the RP model.

However, in the case of Levy matrices, the distribution of eigenvalues has polynomial tails [33], their spectrum is not bounded, and the semicircle law does not apply. As mentioned above, this situation leads to a broad distribution of PT rates. There exist statistically significant clusters of states of a relatively small size that are populated faster than the typical case, because the corresponding classical bit strings are located closer to each in Hamming distance than the typical interstate separation. At first glance, this tendency is counter to the Grover scaling (144). We note, however, that fluctuations of $\Sigma^{\prime}$ and $\Sigma^{\prime \prime}$ are correlated with each other. Faster decay of a marked state also corresponds to a bigger self-energy shift, which reduces the likelihood of finding a marked state with its energy inside the target window $\Delta \mathcal{E}_{\mathrm{cl}} \sim \sum_{*}^{\prime \prime}$.

However, the Grover scaling still survives in a typical case corresponding to the PT away from a randomly selected bit string. For Levy matrices [33], it reflects the fact that the typical width $\Sigma_{\text {typ }}^{\prime \prime}$ of the curve of the global density of states along the energy axis must scale as a square root of the corresponding typical number of states (area under the curve).

\section{COMPARISON WITH THE ANALOG GROVER SEARCH}

\section{A. Grover search starting from a fully symmetrized state}

So far, we have studied the PT protocol with the Hamiltonian (1) $H=H_{D}+H_{\mathrm{cl}}$ that starts from a given marked state of an IB model $H_{\mathrm{cl}}(3)$ and aims at finding a different marked state inside a given window of energies using a transverse-field Hamiltonian $H_{D}=-B_{\perp} \sum_{m=1}^{n} \sigma_{m}^{x}$ (1) as a driver.

We consider here a different protocol inspired by the Hamiltonian version of the Grover algorithm proposed in Ref. [46]. The new protocol finds marked states in the IB model $H_{\mathrm{cl}}$ starting from the ground state of $H_{D}$, which is a fully symmetric state $|S\rangle=2^{-n / 2} \sum_{j=1}^{n}|z\rangle$ in a computational basis. This protocol can be implemented by adjusting the value of transverse field $B_{\perp} \approx 1$ so that the ground-state energy of the driver is set near the center of the IB. Then, we can replace the full driver with the projector on its ground state: $H_{D} \rightarrow-n B_{\perp}|S\rangle\langle S|$. The quantum evolution is guided by the Hamiltonian:

$$
H_{G}=-n B_{\perp}|S\rangle\left\langle S\left|+\sum_{j=1}^{M} \mathcal{E}\left(z_{j}\right)\right| z_{j}\right\rangle\left\langle z_{j}\right| .
$$

With the initial condition, $|\psi(0)\rangle=|S\rangle$. In the case where all impurity energies are equal to each other, $\left\{\mathcal{E}\left(z_{j}\right)=\right.$ $-n\}_{j=1}^{M}$ and $B_{\perp}=1$, the Hamiltonian $H_{G}$ is a generalization of the analog version of a Grover search [46] for the case of $M$ target states. The system performs Rabi oscillations between the initial state $|S\rangle$ and the state which is an equal superposition of all marked (solution) states. The time to solution is the half-period of the oscillations, the "Grover time" $t_{G}$ :

$$
t_{G}=\frac{\pi}{2 n B_{\perp}} \sqrt{\frac{2^{n}}{M}} .
$$

Hamiltonian versions of a Grover search with a transversefield driver whose ground state is tuned at resonance with that of the solution state are considered in Refs. [65,66].

The robustness of the Grover algorithms to phase noise was considered previously in the case of a single marked 
state $[67,68]$. Here, we investigate the role of systematic phase errors in quantum oracle for the case of multiple solutions by assuming that marked state energies take distinct values $\mathcal{E}\left(z_{j}\right)=-n+\epsilon_{j}$ randomly distributed over some narrow range $W$. We also investigate the systematic error in the Grover diffusion operator [1]. In the Hamiltonian formulation [46], this error corresponds to the deviation from unity of the parameter $B_{\perp}$ that controls the weight of the driver in Eq. (146). We define

$$
B_{\perp}=1-\frac{\epsilon_{0}}{n},
$$

where $\epsilon_{0}$ is the driver error.

We denote the computational basis states as $|j\rangle \equiv\left|z_{j}\right\rangle$ with $j \in[1, N], N=2^{n}$, and assume that marked states correspond to the range $j \in[1, M]$. We also introduce the state $|0\rangle=(1 / \sqrt{N-M}) \sum_{j=M+1}^{N}|j\rangle$ that is orthogonal to all the marked states. The subset of basis vectors $\mathcal{S}=$ $\{|j\rangle\}_{j=0}^{M}$ spans the $M+1$-dimensional subspace with the remaining set $\mathcal{S}_{\perp}$ of basis vectors spanning the orthogonal $N-M-1$-dimensional subspace. One can show that $H_{G}$ does not have matrix elements that couple $\mathcal{S}$ with $\mathcal{S}_{\perp}$.

Assuming that $N \gg M$, one can consider the decay of the state $|0\rangle$ instead of the state $|S\rangle$. We use Eq. (147) and omit constant terms and small corrections $\mathcal{O}(M / N)$ in $H_{G}$. The nonzero matrix elements $H_{G}^{i j}=\left\langle i\left|H_{G}\right| j\right\rangle$ in this subspace $\mathcal{S}$ have the form

$$
H_{G}^{j j}=\epsilon_{j}, \quad H_{G}^{j 0}=-\left(1-\delta_{j 0}\right) V, \quad V=n 2^{-n / 2},
$$

where $j \in[0, M]$ and $H_{G}^{j 0}=H_{G}^{0 j}$. On a timescale $t \ll$ $1 / \delta \epsilon=M / W$ much smaller than the inverse spacing of the energies $\epsilon_{j}$, the quantum evolution with initial condition $|\psi(0)\rangle=|0\rangle$ corresponds to the decay of the discrete state with energy $\epsilon_{0}$ into the continuum [60] with the finite spectral width $W$ [69]. It is a similar problem to that discussed in Sec. VIII.

\section{Sensitivity to systematic oracle phase error}

We first consider the case of relatively large oracle errors (wide energy band $W$ )

$$
V \sqrt{M} \ll W \ll V M
$$

and modest driver errors

$$
\epsilon_{0}=n\left(1-B_{\perp}\right) \lesssim W .
$$

In this case, following the results of Sec. VIII on the solution of the Fano-Anderson model [69], we obtain an exponential decay of the initial amplitude [cf. Eq. (79)]

$$
\psi_{0}(t) \simeq \exp \left[-\Sigma_{0}^{\prime \prime} t-i \epsilon_{0} t-i \Sigma_{0}^{\prime}\left(\epsilon_{0}+i 0^{+}\right) t\right]
$$

where $\Sigma_{0}(z)=\Sigma_{0}^{\prime}(z)+i \Sigma_{0}^{\prime \prime}(z)$ is a self-energy and

$\Sigma_{0}(z)=V^{2} \sum_{m=1}^{M} \frac{1}{z-\epsilon_{m}}, \quad \Sigma_{0}^{\prime \prime} \equiv \frac{1}{2} \Gamma_{0}=\frac{\pi V^{2}}{W / M}$.

The state $|0\rangle$ undergoes an exponential decay with the rate $\Gamma_{0}=2 \Sigma_{0}^{\prime \prime}$. After the characteristic time $t_{\mathrm{PT}} \sim 1 / \Gamma_{0}$, the population is transferred into a subset of the marked states with energies inside the window $\left|\epsilon_{j}-\epsilon_{0}\right| \simeq \Sigma_{0}^{\prime \prime} \ll W$.

The number of marked states (solutions) to which the population is transferred is $\Omega \sim \Sigma_{0}^{\prime \prime} / \delta \epsilon$. The relation between $t_{\mathrm{PT}}$ and $\Omega$ is

$$
t_{\mathrm{PT}} \sim \frac{1}{V \sqrt{\Omega}}, \quad \Omega \sim\left(\frac{V}{W / M}\right)^{2},
$$

the same as in the Grover algorithm (146). It also recovers the scaling with $\Omega$ and $n$, up to a factor $\exp \left[-n /\left(2 B_{\perp}{ }^{2}\right)\right]$, for the time of PT considered in the rest of this paper that uses a transverse field as a driver and starts from any marked state instead of a fully symmetric state.

To characterize the effect of oracle errors, we introduce the scaling ansatz for the marked states bandwidth $W \sim$ $2^{-n / 2} M^{\gamma / 2}$ similar to that in Eq. (75). We observe that the number $\Omega$ of solution states populated over the time $t_{\mathrm{PT}}$ cannot be greater than $M$ by construction. For $W \lesssim V \sqrt{M}$ (or $\gamma<1$ ), the value of $\Omega \simeq M$ and the scaling of the transfer time $t_{\mathrm{PT}}$ with $M$ are the same as $t_{\mathrm{G}}$ in the ideal Grover algorithm (146). In the region given by Eq. (149) (or $2>\gamma>1$ ), the algorithm performance is degraded, because $\Omega \ll M$. For $W \gg V M$ (or $\gamma>2$ ), the algorithm fails to find even one solution.

\section{Sensitivity to the systematic driver error}

We now consider the sensitivity of the algorithm to an error in the weight of the driver Hamiltonian, i.e., to the nonzero value of the parameter $e_{0}=n\left(1-B_{\perp}\right)$ [Eq. (147)]. We assume that $\epsilon_{0} \gg W$ while the spread of the marked state energies the condition (149), so that, absent driver errors, the PT time follows a Grover-like scaling law [Eq. (153)].

In this case, the state $|0\rangle$ is coupled nonresonantly to a continuum with a narrow bandwidth. The expression for the population transfer to the marked states can be obtained from the time-dependent perturbation theory in the parameter $\epsilon_{0} / W$ :

$$
\sum_{m=1}^{M}\left|\psi_{m}(t)\right|^{2}=\frac{2 M V^{2}}{\epsilon_{0}^{2}}\left(1-\cos \left(\epsilon_{0} t\right) \frac{\sin (W t / 2)}{W t / 2}\right) .
$$

Maximum transfer occurs at the time $t_{0}=\pi / \epsilon_{0}$ with the total transferred probability $p_{0}=4 M V^{2} / \epsilon_{0}^{2}$. The typical time $t_{\mathrm{PT}} \simeq t_{0} / p_{0}$ to achieve the successful population 
transfer to marked states involves repeating the experiment $1 / p_{0}$ times:

$$
t_{\mathrm{PT}}=\frac{1}{\Gamma_{0}} \frac{\pi^{2} \epsilon_{0}}{W},
$$

where $\Gamma_{0}$ is given in Eq. (152) and the first multiple in the rhs gives the typical transfer time in the absence of driver errors. The latter leads to an increase of the transfer time by a large factor of $\epsilon_{0} / W$.

For the maximum possible bandwidth $W$ when nearly all states are populated, $W \sim \Gamma_{0} \sim V \sqrt{M}$, the time of population transfer [Eq. (154)] is

$$
t_{\mathrm{PT}} \sim t_{G}\left(t_{G} \epsilon_{0}\right) \quad\left(\epsilon_{0} \gg t_{G}^{-1} \sim V \sqrt{M}\right) .
$$

As expected, when the driver error exceeds inverse Grover time $1 / t_{G}$, the performance of analog Grover algorithms (145) degrades relative to $t_{G}$. This degradation is a direct consequence of the fact that the quantum evolution begins from a fully symmetric state which is a ground state of the driver Hamiltonian whose energy is tuned at resonance with the marked states. In this case, the transverse-field Hamiltonian driver effectively corresponds to the projector (145). Because the ground state is not degenerate, the resonance region is exponentially narrow $\left(\sim 2^{-n / 2} \sqrt{M}\right)$, which results in the exponential sensitivity of the Grover algorithm performance to the value of the driver weight. This critical behavior is studied in the work on quantum spatial search [70] for the case of one marked state.

In contrast, in the PT protocol considered earlier in this paper, there is no need to fine-tune the value of $B_{\perp}$ other than making it large, $B_{\perp} \gg 1$, which happens because the effective coupling between the marked states described by the down-folded Hamiltonian $\mathcal{H}$ (41) is not due to any one particular eigenstate of the driver (such as the state $|S\rangle$ for the Grover case). Instead, this coupling is formed due to an exponentially large (in $n$ ) number of nonresonant, virtual transitions between the marked states and highly exited states of the transverse-field Hamiltonian $H_{D}$. This coupling results in a significant improvement in robustness for the proposed PT relative to the analog Grover algorithm.

\section{B. Grover search starting from a marked state}

We now consider an implementation of the analog Grover search that starts from the marked state similar to the PT protocol considered in previous sections. The transition amplitude $U_{i j}(t)=\left\langle i\left|\exp \left(-i H_{G} t\right)\right| j\right\rangle$ between the two marked states can be written in the form

$$
U_{j i}(t)=\sum_{\lambda} e^{-i \lambda t} \psi_{\lambda}(i) \psi_{\lambda}(j)
$$

Here, $\psi_{\lambda}(j)=\left\langle j \mid \psi_{\lambda}\right\rangle$ are amplitudes of the eigenstates of $H_{G}$ in the $M+1$-dimensional subspace, and $\lambda$ are the corresponding eigenvalues that obey the equation

$$
\lambda=\epsilon_{0}+\sum_{j=1}^{M} \frac{V^{2}}{\lambda-\epsilon_{j}}, \quad \psi_{\lambda}(j)=\frac{V}{\lambda-\epsilon_{j}} \frac{1}{\sqrt{Z_{\lambda}}} .
$$

Here,

$$
Z(\lambda)=1+\sum_{m=0}^{M} \frac{V^{2}}{\left(\lambda-\epsilon_{m}\right)^{2}} .
$$

Instead of providing a detailed analysis of the above solution, we provide an order of magnitude estimate to extract the relevant scaling behavior. We again assume that the spread of the marked state energies, $W=t_{G}^{-1}=$ $\mathcal{O}(V \sqrt{M})$, corresponds to the inverse of the Grover time $t_{G}$ needed to find any one of the solutions with equal probability. The typical separation between the adjacent values of $\epsilon_{j}$ is $\delta \epsilon=W / M \sim V / \sqrt{M}$.

It follows from Eq. (157) that in the ordered array obtained by combining together the sets of energies $\left\{\epsilon_{j}\right\}_{j=0}^{M}$ and eigenvalues $\left\{\lambda_{m}\right\}_{m=0}^{M}$ their values appear alternatively and sequentially, e.g., $\epsilon_{j-1}<\lambda_{j}<\epsilon_{j}<\lambda_{j+1}$. The typical separation between the adjacent elements in the array is $\left|\lambda_{j}-\epsilon_{j}\right| \sim \delta \epsilon$. We observe that for a given value of $\lambda$ the sum in the expression for $Z(\lambda)$ [Eq. (158)] is dominated by the small, $\mathcal{O}(1)$, number of terms with $\left|\epsilon_{m}-\lambda\right| \sim \delta \epsilon$, each term of the order of $M$. Indeed, there are $\mathcal{O}(M)$ remaining terms corresponding to $\left|\epsilon_{m}-\lambda\right| \sim W$. The magnitude of those terms is $V^{2} / W^{2} \sim 1 / M$, and their aggregated contribution to the sum is $\mathcal{O}(1)$. Therefore, we can estimate $Z(\lambda)=\mathcal{O}(M)$, and for the amplitudes we have

$$
\psi_{\lambda}(m) \sim \frac{V}{\lambda-\epsilon_{m}} \frac{1}{\sqrt{M}}, \quad m=i, j .
$$

For a given initial state $|i\rangle$ at time $t$, we pick the final state $|j\rangle$ within the energy window $\epsilon_{i}-\epsilon_{j} \sim \Delta=1 / t$ around $\epsilon_{i}$. The sum in the expression (156) for the transition amplitude $U_{j i}(t)$ is dominated by the number of terms $\Omega=$ $\Delta / \delta \epsilon \sim \Delta \sqrt{M} / V$ corresponding to the eigenvalues $\lambda$ inside the same window of energies. For those terms $\lambda-\epsilon_{i}, \epsilon_{j}-$ $\lambda \sim \Delta$ giving the estimate for the amplitudes $\psi_{\lambda}(i), \psi_{\lambda}(j) \sim$ $1 / \Omega$ [cf. Eq. (159)]. The magnitude of the sum in Eq. (156) can be estimated as $\left|U_{i j}(t)\right| \sim \Omega\left|\psi_{\lambda}(i) \psi_{\lambda}(j)\right| \sim 1 / \Omega$. On the other hand, because ordered values of $\lambda$ and $\epsilon_{m}$ alternate in sequence, the probability $\left|U_{i j}(t)\right|^{2}$ is distributed over $\Omega$ marked states and $\left|U_{i j}(t)\right| \sim \Omega^{-1 / 2}$. By equating the above two estimates for $\left|U_{i j}(t)\right|$, we immediately obtain $\Omega \sim 1$ and, therefore, 


$$
\Delta=\frac{1}{t} \sim \delta \epsilon \sim \frac{V}{\sqrt{M}}
$$

In the case when there are only a few marked states $(M \sim 1$ and $W \sim V)$, the probability is initially localized on a given marked state $|i\rangle$, and then it spreads over to other states separated in energy by $V$ during the time $t_{G} \sim 1 / V \sim 2^{n / 2}$. In this case, the algorithm time scales with $n$ identically to that of the analog Grover search that starts at the fully symmetric state $|S\rangle$. Similar performance is achieved by the PT protocol using transverse field $B_{\perp} \gg 1$ and discussed in previous sections.

The difference from an analog Grover search starting at $|S\rangle$ from the above PT protocol using a transverse field becomes dramatic for a large number of marked states $M \gg 1$. Both an analog Grover search and the PT protocol benefit from the increase in $M$ : The algorithmic time shrinks $\propto 1 / \sqrt{M}$, and the number of marked (solution) states $\Omega$ in the number of states in the final superposition increases with $M$.

In contrast, the quantum search with $H_{G}$ starting form the marked state $|i\rangle$ does not create massive superpositions of solution states when $M$ increases. Instead, it involves a very few other states that are adjacent in energy, $\left|\epsilon_{j}-\epsilon_{i}\right| \sim V / \sqrt{M}$. The time of the algorithm increases with $M$ [Eq. (160)]. This increase happens because, unlike the Hamiltonian $H$ with a transverse field [Eq. (1)], the Hamiltonian $H_{G}$ is integrable. The wave function remains localized near the initial marked state.

\section{CONCLUSION}

In this paper, we developed the first well-controlled theoretical description of the eigenstate structure and quantum dynamics in the NEE phase in a quantum spin glass. The distinctive feature of a NEE in quantum spin glass is that the eigenstates are formed by coherent superpositions of a large number of local minima separated by extensive Hamming distances. The local minima are connected by quantum tunneling matrix elements corresponding to a sum over a large number of virtual under the barrier trajectories. Calculating such matrix elements is challenging, because contributions from different trajectories interfere with each other; technically, this interference means that looped paths have to be included in the perturbation theory expansion, making it difficult to work with. Moreover, perturbation expansion diverges in the delocalized phase. In this paper, we overcome these difficulties by developing an asymptotically exact WKB description of the tunneling matrix elements and, subsequently, a wellcontrolled cavity equation approach to describe delocalized nonergodic states.

We develop this approach considering the IB model with a "bimodal" energy function: $\mathcal{E}(z)=0$ for all states except for $M$ "marked" states $\left|z_{j}\right\rangle$ picked at random with energies forming a narrow band of the width $W$ separated by a large gap $\mathcal{O}(n)$ from the rest of the states. At a zero transverse field, this classical model demonstrates the frozen (replica symmetric) spin-glass phase characterized by Edwards-Anderson order parameter $q_{\mathrm{EA}}=1$ below the transition temperature $\beta_{f}=\ln 2-(1 / n) \ln M$. The well-controlled theory of the statistics of matrix elements allows us to uncover asymptotic orthogonality between the subspace of marked states and the rest of the Hilbert space, a qualitatively new feature of the spectrum that cannot be obtained by the leading-order perturbation theory in a transverse field. As a result, the subspace of marked states can be described with an effective downfolded $M \times M$ Hamiltonian $\mathcal{H}$ that is dense in the space of the marked states $\left|z_{j}\right\rangle$. Its off-diagonal matrix elements $\mathcal{H}_{i j}=V\left(d_{i j}\right) \cos \phi\left(d_{i j}\right)$ depend only on the Hamming distance $d$ and are obtained using the WKB method. The distribution of matrix elements $\mathcal{H}_{i j}$ has a heavy tail decaying as a cubic power for $V(d) \gg V_{\text {typ }}$. This tail is a remarkable result of the competition between the very steep decay of the off-diagonal tunneling matrix element with the Hamming distance $d$ and the steep increase in the number of marked states $M_{d} \propto\left(\begin{array}{l}n \\ d\end{array}\right)$ at distance $d$. We emphasize that such a polynomial tail in the distribution of matrix elements is possible only either in an infinite dimension or in the presence of long-range interactions (e.g., dipolar glass).

The dispersion of the diagonal elements $\mathcal{H}_{j j}=\mathcal{E}\left(z_{j}\right)$ is expected to be large: $W \sim V_{\text {typ }} M^{\gamma / 2} \gg V_{\text {typ }}$ with $\gamma \in[1,2]$. Therefore, we call $\mathcal{H}_{i j}$ a preferred basis Levi matrix (PBLM), a generalization of the Levi matrix from the random matrix theory. We demonstrate two localization transitions in the PBLM ensemble whose locations are determined by the strong hierarchy of elements of the PBLM $\mathcal{H}_{i j}$. In the range $1<\gamma<2$, there exist minibands of nonergodic delocalized eigenstates of $\mathcal{H}$. Their width is proportional to $1 / t_{\mathrm{PT}} \ll W$. Each miniband associated with a support set $\mathcal{S}$ over the marked states. If $\gamma>2$, then $W$ exceeds the largest matrix element of $\mathcal{H}_{i j}$ and the support set is empty-all eigenstates are localized. If $\gamma<1$, then $W$ is smaller than the typical largest element in a row of $\mathcal{H}_{i j}$ and the support set extends to all marked states - all eigenstates are "ergodic" within the subspace of marked states.

We find the distribution of the miniband width $\Gamma$ analytically by solving the nonlinear cavity equations for an ensemble of PBLMs. Unlike previous analyses focused on linearized cavity equations near the Anderson transition, we find the solution of the fully nonlinear cavity equations in the nonergodic delocalized phase. The distribution of miniband widths $\Gamma$ obeys the alpha-stable Levi law with tail index 1 . The typical value of $\Gamma$ and its characteristic variance exceeds the typical matrix element of $\mathcal{H}$ by a factor of $\Omega^{1 / 2}$, where $\Omega=\left(M V_{\text {typ }} / W\right)^{2}$ is the size of the support set in a typical miniband. 
The novel features of the quantum spin-glass dynamics described in this paper have important implications for quantum search and optimization algorithms. Specifically, coherent multiqubit tunneling gives rise to minibands of nonergodic delocalized quantum states which provide a coherent pathway for PT between computational states with close energies in a spin-glass energy landscape. Dynamics in the NEE regime cannot be efficiently simulated by QMC, in contrast to sequential tunneling often arising in quantum annealing algorithms.

We define a computational primitive with the objective to find bit strings $z_{j} \neq z_{i}$ inside some narrow energy window $\Delta \mathcal{E}_{\mathrm{cl}}$ around the energy of the initial bit string $z_{i}$. The problem is hard for sufficiently low starting energy $\mathcal{E}\left(z_{i}\right)$ in the region proliferated by deep local minima that are separated by large Hamming distances. This landscape is similar to that in an analog Grover search [46,65] with multiple target states and a distribution of oracle values for the targets. The best-known classical algorithm for finding another marked state has cost $O\left(2^{n} / M\right)$.

We propose to solve this problem using the following quantum PT protocol: Prepare the system in a computational state $\left|z_{j}\right\rangle$ with classical energy $\mathcal{E}\left(z_{j}\right)$ and then evolve it with the transverse-field quantum spin Hamiltonian. Classical energies $\mathcal{E}(z)$ are encoded in the problem Hamiltonian diagonal in the basis of states $|z\rangle$ similar to quantum annealing (QA) approaches [2-4]. A key difference from QA or analog quantum search Hamiltonians $[46,70]$ is that the transverse field is kept constant throughout the algorithm and is not fine-tuned to any particular value. At the final moment of PT, we projectively measure in the computational basis and check if the outcome $z$ is a "solution," i.e., $z \neq z_{j}$, and the energy $\mathcal{E}(z)$ is inside the window $\Delta \mathcal{E}_{\mathrm{cl}}$.

We demonstrate that for the IB model quantum PT finds another state within a target window of energies $\Omega$ in time $t_{\mathrm{PT}} \propto 2^{n / 2} \Omega^{-1 / 2} \exp \left[n /\left(2 B_{\perp}^{2}\right)\right]$. The scaling exponent of $t_{\mathrm{PT}}$ with $n$ differs from that in Grover's algorithm by a factor of $\propto B_{\perp}^{-2}$, which can be made small with large transverse fields $n \gg B_{\perp}^{2} \gg 1$.

Crucial distinctions between this case and the Hamiltonian in the analog version of Grover's algorithm [46] for the case of multiple target states are the nonintegrability of our model and the delocalized nature of the eigenstates within the energy band $W$. Furthermore, the analog Grover's algorithm for multiple targets is exponentially sensitive in $n$ to the weight of the driver Hamiltonian and cannot be initialized with a computational basis state.

The model (1) considered in this paper belongs to the class of $n$-local infinite range spin glasses similar to the quantum random energy model in a transverse field [71]. However, the key feature of our analysis - transport via a miniband of nonergodic delocalized states at the tail of the density of states dominated by deep local minima - is ubiquitous to a broad class of quantum spinglass models (1), such as transverse-field Sherrington Kirkpatrick, $p$-spin model [39], $K$ satisfiability, etc.

In the above models, one can identify two distinct energy scales. The second scale is the typical width of nonergodic minibands, $\Gamma \sim V^{2} \rho$, determined by typical values of matrix element $V$ between low-energy states and the density of low-energy states $\rho$. The delocalized low-energy states satisfying $V \rho>1$ form minibands that are nonergodic, i.e., are characterized by an exponentially small width and, therefore, an exponentially small fraction of density of states contributing to a single miniband $V^{2} \rho \sim \exp (-\varphi n)$ with an exponent $0<\phi<1$. The tunneling transitions between the states inside the miniband require a large number of spin flips, and, therefore, $\mathcal{E}_{\text {flip }} \gg \Gamma$. Starting from the initial state $\left|z_{i}\right\rangle$ inside the strip of energies $\Delta \mathcal{E}_{\mathrm{cl}}$, the quantum evolution is confined within the corresponding miniband. The quantum PT can be described by an effective down-folded Hamiltonian $\mathcal{H}_{i j}$ defined over a subset of computational basis states whose classical energies lie within the energy strip $\Delta \mathcal{E}_{\mathrm{cl}}$ at the tail of the density of states. We also note that analytical [72] and numerical [73] analyses of nonergodic delocalized states in random energy model have been recently reported.

\section{ACKNOWLEDGMENTS}

The authors are grateful to Edward Farhi, Lev Ioffe, Vladimir Kravtsov, Christopher Laumann, and Antonello Scardicchio for the fruitful discussions of this work. K. K. acknowledges support by NASA Academic Mission Services, Contract No. NNA16BD14C. This research is based upon work supported in part by the AFRL Information Directorate under Grant No. F4HBKC4162G001 and the Office of the Director of National Intelligence (ODNI) and the Intelligence Advanced Research Projects Activity (IARPA), via IAA 145483. The views and conclusions contained herein are those of the authors and should not be interpreted as necessarily representing the official policies or endorsements, either expressed or implied, of ODNI, IARPA, AFRL, or the U.S. Government. The U.S. Government is authorized to reproduce and distribute reprints for Governmental purpose notwithstanding any copyright annotation thereon.

\section{APPENDIX A: MATRIX ELEMENTS OF THE DOWN-FOLDED HAMILTONIAN AND THE NORMALIZATION CONDITION FOR ITS EIGENVECTORS}

We introduce eigenstates $|x\rangle$ of the transverse-field (driver) Hamiltonian

$$
H_{D}=-B_{\perp} \sum_{j=0}^{n} \sigma_{j}^{x}=\sum_{x \in\{0,1\}^{n}} H_{D}^{x}|x\rangle\langle x| .
$$


Here,

$$
|x\rangle=\left|x^{1}\right\rangle \otimes \cdots \otimes\left|x^{n}\right\rangle,
$$

where $\left|x^{k}\right\rangle$ is the state of the $k$ th qubit such that $\sigma_{x}\left|x^{k}\right\rangle=$ $\left(1-2 x_{k}\right)\left|x^{k}\right\rangle$ and $x$ bits take values $x^{k}=0,1$. Also,

$$
H_{D}^{x}=-B_{\perp}\left(n-2 h_{x}\right), \quad h_{x}=\sum_{k=1}^{n} x^{k}
$$

where $h_{x}$ is a Hamming weight of the bit string $x$ and $-B_{\perp}(n-2 h)$ and $h \in(0, n)$ are eigenvalues of $H_{D}$.

We expand the eigenstates $|\psi\rangle$ of the system Hamiltonian $H$ (1) into the basis of the eigenstates $|x\rangle$ :

$$
|\psi\rangle=\sum_{x \in\{0,1\}^{n}} \Psi(x)|x\rangle
$$

We write the Schrödinger equation $H|\psi\rangle=E|\psi\rangle$ in the form

$$
H_{D}|\psi\rangle+\sum_{j=1}^{M} \mathcal{E}\left(z_{j}\right)\left|z_{j}\right\rangle \psi\left(z_{j}\right)=E|\psi\rangle,
$$

where $\psi\left(z_{j}\right)=\left\langle z_{j} \mid \psi\right\rangle$. Then, we multiply it from the left by $\langle x|$ and obtain $\Psi(x)$ in terms of $\psi\left(z_{j}\right)$ :

$$
\Psi(x)=\frac{\sum_{j=1}^{M} \mathcal{E}\left(z_{j}\right) v_{x, j} \psi\left(z_{j}\right)}{E-H_{D}^{x}} .
$$

In Eq. (A6), the coefficients $v_{x, j}=\left\langle x \mid z_{j}\right\rangle$ equal

$$
v_{x, j}=2^{-n / 2}(-1)^{x \cdot z_{j}}, \quad x \cdot z_{j} \equiv \sum_{k=1}^{n} x^{k} z_{j}^{k},
$$

and $z_{j}^{k}=0,1$.

We now multiply Eq. (A5) from the left by $\left\langle z_{j}\right|$, where $j \in(1, M)$ enumerates marked states, and obtain

$$
\sum_{x \in\{0,1\}^{n}} H_{D}^{x} \Psi(x) v_{x, j}=\left[E-\mathcal{E}\left(z_{j}\right)\right]\left\langle z_{j} \mid \psi\right\rangle .
$$

Plugging here the expression for $\Psi(x)$ [Eq. (A6)] into the matrix eigenvalue problem [Eq. (A5)], we obtain

$$
\mathcal{E}\left(z_{i}\right) \psi\left(z_{i}\right)-\sum_{j=1}^{M} \mathcal{E}\left(z_{j}\right) c_{i j}(E) \psi\left(z_{j}\right)=E \psi\left(z_{i}\right),
$$

where

$$
c_{i j}(E)=\sum_{x \in\{0,1\}^{n}} v_{x, i} v_{x, j} \frac{H_{D}^{x}}{E-H_{D}^{x}}
$$

Because $H_{D}^{x}$ depends on a bit string $x$ only via its Hamming weight $\sum_{j=1}^{n} x^{j}$, one can perform the partial summation in Eq. (A10) to get

$c_{i j}(E) \equiv c\left(E,\left|z_{i}-z_{j}\right|\right), \quad\left|z_{i}-z_{j}\right|=\sum_{k=1}^{n}\left|z_{i}^{k}-z_{j}^{k}\right|$,

where the function $c(E, d)$ has the form

$c(E, d)=\sum_{k=0}^{n-d} \sum_{l=0}^{d}\left(\begin{array}{l}n \\ k\end{array}\right)\left(\begin{array}{c}n-d \\ l\end{array}\right) \frac{(-1)^{l} 2^{-n}}{1+\frac{E}{B_{\perp}(n-2 k-2 l)}}$.

Above, $\left|z_{i}-z_{j}\right|$ denotes the Hamming distance between the bit strings $z_{i}$ and $z_{j}$. We introduce the rescaling

$$
\psi\left(z_{i}\right)=\frac{A_{i}}{\sqrt{\mathcal{E}\left(z_{i}\right)}}, \quad i \in[1 \ldots M] .
$$

Then, Eq. (A9) can be written in the form

$$
\sum_{j=1}^{M} \mathcal{H}_{i j}(E) A_{j}=E A_{i}
$$

where $\mathcal{H}_{i j}$ is a symmetric $M \times M$ matrix

$$
\mathcal{H}_{i j}(E)=\delta_{k j} \mathcal{E}\left(z_{i}\right)+\sqrt{\mathcal{E}\left(z_{i}\right) \mathcal{E}\left(z_{j}\right)} c\left(E, d_{i j}\right),
$$

indices $k, j=1: M$, and $\delta_{k j}$ is the Kronecker delta. This nonlinear eigenproblem is given in the main text [Eq. (18)].

We note that the projections of the eigenvectors of $H$ onto the marked state subspace are not, in general, normalized, nor are they orthogonal. Let us consider the eigenstate $\left|\psi_{\beta}\right\rangle$ and the corresponding eigenvalue $E_{\beta}$ of $H$. We calculate the corresponding amplitude $\Psi_{\beta}(x)$ using Eq. (A6) and plug it into the normalization condition

$$
\sum_{x \in\{0,1\}^{n}} \Psi_{\beta}^{2}(x)=1,
$$

obtaining after partial summation

$$
\sum_{i, j=1}^{M} \mathcal{E}_{i} \mathcal{E}_{j} r\left(E_{\beta}, d_{i j}\right) \psi_{\beta}\left(z_{i}\right) \psi_{\beta}\left(z_{j}\right)=1,
$$

where the coefficient $r(E, d)$ equals

$$
r(E, d)=2^{-n} \sum_{k=0}^{n-d} \sum_{l=0}^{d} \frac{(-1)^{k}\left(\begin{array}{c}
d \\
k
\end{array}\right)\left(\begin{array}{c}
n-d \\
l
\end{array}\right)}{B_{\perp}[n-2(k+l)+E]^{2}} .
$$

It can be written in the form 


$$
r(E, d)=\frac{\partial}{\partial E}\left(\frac{c(E, d)-\delta_{d, 0}}{E}\right),
$$

where $\delta_{d, 0}$ is the Kronecker delta. We use Eq. (A15) and write

$$
r\left(E, d_{i j}\right)=\frac{1}{\sqrt{\mathcal{E}_{i} \mathcal{E}_{j}}} \frac{\partial \mathcal{H}_{i j}(E)}{\partial E} .
$$

We now define the coefficients $Q_{i j}(E)$ such that

$$
\frac{1}{Q_{i j}(E)}=\mathcal{E}_{i} \mathcal{E}_{j} r\left(E, d_{i j}\right)=\sqrt{\mathcal{E}_{i} \mathcal{E}_{j}} \frac{\partial \mathcal{H}_{i j}(E)}{\partial E}
$$

Then, Eq. (A17) takes the form

$$
\sum_{i, j} \frac{1}{Q_{i j}(E)} \psi_{\beta}\left(z_{i}\right) \psi_{\beta}\left(z_{j}\right)=1 .
$$

The above equations (A21) and (A22) correspond to Eqs. (21) and (22) of the main text.

\section{APPENDIX B: DETAILS OF THE WKB ANALYSIS OF THE COUPLING COEFFICIENTS}

In the main text, we express the coupling coefficient $c(E, d)$ in terms of the off-diagonal matrix elements of the resolvent (12) of the transverse-field Hamiltonian $H_{D}$ between the states that belong to a maximum total spin subspace $S=n / 2$. The results are given in the expressions (29) and (30) from the main text repeated below for convenience:

$$
c(E, d)=\delta_{d, 0}-\frac{E}{\sqrt{\left(\begin{array}{l}
n \\
d
\end{array}\right)}} G_{(n / 2)-d,(n / 2)}(E) .
$$

Here, the resolvent $G_{(n / 2)-d,(n / 2)}(E)$ obeys the inhomogeneous equation

$$
\begin{array}{r}
\delta_{m,(n / 2)}+\sum_{s= \pm 1} u(m-s / 2) G_{m+s,(n / 2)}=E G_{m,(n / 2)}, \\
u(m)=-B_{\perp} \sqrt{L^{2}-m^{2}}, \quad L=\frac{n+1}{2} .
\end{array}
$$

We solve the above equations for the case where the energy $E$ of the resolvent is not far from the center of the impurity band:

$$
E=-n+\Delta, \quad \Delta=\mathcal{O}\left(n^{0}\right) .
$$

The WKB solution to Eq. (B2) is sought in the exponential form

$$
G_{m,(n / 2)} \propto \exp \left(i \int^{m} d k p(k)\right)
$$

It is assumed that $\int_{0}^{m} d k p(k)=\mathcal{O}(n)$ and $|p(k)|=\mathcal{O}\left(n^{0}\right)$ so that $G_{m,(n / 2)}$ is varying steeply with $m$ changing by 1 . However, $\left|p^{\prime}(m)\right|=\mathcal{O}(1 / n)$, and $p(m)$ is varying very slowly with $m$ due to the similar property of the coefficients $u(m) / L$ in Eq. (B2). This property is at the root of the WKB approximation [57]. The quantity $p$ corresponds to the "momentum" of the effective mechanical system with coordinate $m$, energy $E$, and Hamiltonian function $u(m) \cos p$. The function $p=p(E, m)$ is obtained from the equation

$$
u(m) \cos p=E .
$$

This equation also defines the curve on the $(m, E)$ plane with $p=0$ shown in Fig. 3. Points on that curve are turning points of the classical motion with energy $E$.

For not too small transverse fields

$$
B_{\perp}>\frac{2 L}{|E|} \simeq 1
$$

Eq. (B6) has two types of WKB solution that correspond to real or imaginary momentum $p(m)$ depending on the value of $m$ relative to the turning points $m= \pm m_{0}(E)$ given below [74]:

$$
m_{0}=\sqrt{L^{2}-\left(\frac{E}{2 B_{\perp}}\right)^{2}} .
$$

In the region

$$
n / 2+m_{0}>d>n / 2-m_{0}
$$

the amplitude $G_{(n / 2)-d,(n / 2)}$ [Eq. (B5)] is rapidly oscillating with $d$ and can be written in the form

$G_{(n / 2)-d,(n / 2)}=-\mathcal{C}(E) \frac{\sin \phi(E, d)}{\left[m_{0}^{2}(E)-(n / 2-d)^{2}\right]^{1 / 4}}$,

where

$$
\phi(E, d)=\int_{n / 2-d}^{m_{0}} d k \arcsin \left(\sqrt{\frac{m_{0}^{2}-k^{2}}{L^{2}-k^{2}}}\right)-\frac{\pi}{4}
$$

is a phase of the WKB solution and $\mathcal{C}(E)$ is the constant of integration that is discussed below.

On the other hand, in the two regions

$$
d \in\left[0, n / 2-m_{0}\right] \cup\left[n / 2+m_{0}, n\right],
$$


the resolvent $G_{(n / 2)-d,(n / 2)}$ is decreasing exponentially with $d$. For example, in the left region,

$$
G_{(n / 2)-d,(n / 2)}=\frac{\mathcal{C}(E)}{2} \frac{e^{|\operatorname{Im} \phi(E, d)|}}{\left[(n / 2-d)^{2}-m_{0}^{2}(E)\right]^{1 / 4}} .
$$

We omit here for brevity the expression in the right region [Eq. (B12)].

\section{Determination of the integration constant in WKB solution}

Within the WKB approach, the integration constant $\mathcal{C}(E)$ can be obtained by matching the exponential asymptotic (B13) with the solution obtained near the boundary of the interval $d=0$. However, as discussed in Sec. VI B of the main text, for the relevant range of the model parameters, the properties of the typical sample in the ensemble of the IB Hamiltonians $\mathcal{H}$ depend only on $G_{(n / 2)-d,(n / 2)}$ in the region of its oscillatory behavior [Eq. (B9)] away from the boundaries of the interval $d=0, n$. To avoid the analysis in the region of no consequence for us, we determine $\mathcal{C}(E)$ by equating the above WKB asymptotic for $G_{(n / 2)-d,(n / 2)}$ at the center of the interval $d=n / 2$ with an expression for $G_{0,(n / 2)}$ at that point obtained in a different way.

Using Eq. (23), we write $c(E, n / 2)$ in the integral form

$$
c\left(E, \frac{n}{2}\right)=\frac{i E}{2^{n} B_{\perp}} \int_{0}^{\infty} d \tau\left(1-e^{4 i \tau}\right)^{n / 2} e^{i\left(E / B_{\perp}-n+i o\right) \tau}
$$

$(o \rightarrow+0)$. The integral can be expressed in terms of the Gamma function $\Gamma(x)$. In the region of not too small transverse fields [Eq. (B7)], it has the form

$$
c\left(E, \frac{n}{2}\right)=\frac{2^{1-n} \pi a\left(a^{2}-1\right)^{-1} \Gamma\left(\frac{n}{2}\right)}{\sin \left(\frac{\pi(a-1) n}{4 a}\right) \Gamma\left(\frac{(a+1) n}{4 a}\right) \Gamma\left(\frac{(a-1) n}{4 a}\right)},
$$

where

$$
a=-\frac{n B_{\perp}}{E}>1
$$

Using the Sterling formulas for the Gamma function, we obtain in the limit $n \gg 1, a=\mathcal{O}\left(n^{0}\right)$,

$$
\begin{aligned}
& c\left(E, \frac{n}{2}\right)=\frac{\sqrt{n \pi}}{2 a \sin \left(\frac{\pi(a-1) n}{4 a}\right)} 2^{-n / 2} e^{-n \theta(a)}, \\
& \theta(a)=\frac{2 \arctan h\left(\frac{1}{a}\right)+a \ln \left(1-a^{-2}\right)}{4 a} .
\end{aligned}
$$

For large transverse fields, $a \gg 1$, and we have $\theta \simeq a^{2} / 4$.

Using Eq. (B1), we obtain the asymptotic of the Green function at the zone center:
$G_{0,(n / 2)}(E)=\left(\frac{\pi}{8 n^{3}}\right)^{1 / 4} \frac{\exp [-n \theta(a)]}{\sqrt{B_{\perp}^{2}-1} \sin \phi(n / 2, E)}$.

Here, we use the equality for the phase WKB $\phi(E, n / 2)$ [Eq. (B11)] at the zone center:

$$
\phi(E, n / 2)=\pi \frac{(a-1) n}{4 a} .
$$

On the other hand, from the WKB expression (B10), we get

$$
G_{0, \frac{n}{2}}=-\mathcal{C}(E)\left(\frac{2}{n}\right)^{1 / 2} \frac{\sin \phi(E, n / 2)}{\left(1-B_{\perp}^{-2}\right)^{1 / 4}}
$$

By comparing Eqs. (B18) and (B20), we finally obtain the constant of integration $\mathcal{C}(E)$ :

$$
\mathcal{C}(E)=-\frac{\pi^{1 / 4}}{32 n B_{\perp}^{2}\left(B_{\perp}^{2}-1\right)^{1 / 4}} \frac{\exp [-n \theta(a)]}{[\sin \phi(E, n / 2)]^{2}} .
$$

One can use Eq. (B21) in Eqs. (B10) and (29) to obtain the expression for $c(E, d)$ in the region (B9). Before providing the result, we observe that for energies $E$ not too far from the impurity band center [cf. Eq. (B4)] the expression for $n \theta(a)$ can be expanded in powers of $1 / n$ :

$$
n \theta(a) \simeq n \theta\left(B_{\perp}\right)-\frac{E+n}{2 B_{\perp}} \operatorname{arccoth} B_{\perp}+\mathcal{O}\left(n^{-1}\right),
$$

where $E+n \equiv \Delta=\mathcal{O}\left(n^{0}\right)$.

Finally, the expression for the coupling coefficient has the form

$c(E, d)=\sqrt{A(E, d / n)} \frac{n^{\frac{1}{4}} e^{-n \theta\left(B_{\perp}\right)}}{\sqrt{\left(\begin{array}{l}n \\ d\end{array}\right)}} \times \sqrt{2} \sin \phi(E, d)$,

where the WKB phase $\phi(E, d)$ is given in Eq. (B11) and the coefficient $A(E, \rho)$ equals

$$
\begin{gathered}
A(E, \rho)=\sqrt{\frac{\pi}{32}} \frac{e^{\left[(E+n) / B_{\perp}\right] \operatorname{arccoth} B_{\perp}}}{\left(B_{\perp}^{2}-1\right) v(\rho) \sin ^{4}[\phi(E, n / 2)]}, \\
v(\rho)=\left(1-\frac{(1-2 \rho)^{2}}{1-B_{\perp}^{-2}}\right)^{1 / 2} .
\end{gathered}
$$

It is related to $A(\rho)$ in Eq. (43) of the main text as follows: $A(\rho)=A\left(E^{(0)}, \rho\right)$. The phase $\phi(E, n / 2)$ in Eq. (B24) has an explicit form:

$$
\phi(E, n / 2)=\frac{\pi}{4}\left(n\left(1-B_{\perp}^{-1}\right)+\frac{n+E}{B_{\perp}}\right) .
$$




\section{Limit of large transverse fields $B_{\perp} \gg 1$}

In the limit of large transverse fields, the tuning point $m_{0}$ [Eq. (B8)] is very close to the boundary of the interval $m=L$ so that one has a small parameter

$$
\sqrt{\frac{L-m_{0}}{L}}=\frac{1}{\sqrt{8}} \frac{|E|}{L B_{\perp}} \ll 1 .
$$

In this case, the expression for the WKB phase takes a simple form:

$$
\begin{aligned}
& \phi(E, d)=\frac{\pi d}{2}-\frac{\pi n}{4} \frac{\chi(E, d / n)}{B_{\perp}}, \\
& \chi(E, \rho)=\left(1-\frac{\Delta}{n}\right)\left(1-\frac{2}{\pi} \tan ^{-1} \frac{1-2 \rho}{\sqrt{1-(1-2 \rho)^{2}}}\right),
\end{aligned}
$$

where $\Delta=E+n=\mathcal{O}\left(n^{0}\right)$ and values of $d$ are not too close to the interval boundaries:

$$
n-d, d \gg L-m_{0} \sim \frac{n}{B_{\perp}^{2}} .
$$

We note that for large transverse fields $B_{\perp} \gg 1$ the phase is a sum of the two terms. The first term changes rapidly with $d$ with the slope $\pi / 2$, and the second term changes very little [by an amount $\mathcal{O}\left(n^{-1}\right)$ ] when $d$ is changed by 1 .

We note that, unlike the study of the WKB eigenfunctions where one has to select the WKB solution that decays into the classically forbidden region (B12), the Green function $G_{n / 2-d, n / 2}(E)$ corresponds to the solution that increases exponentially with $m=n / 2-d>m_{0}$. Using the oscillating [Eq. (B10)] and exponentially growing [Eq. (B13)] WKB solutions, one can obtain the coefficient $c(E, d)$ from the relation (29). This coefficient provides an asymptotic WKB form of $c(E, d)$ almost everywhere on the interval $d \in[0, n]$ except for the small vicinities of the turning points, $\left|n / 2-m_{0}(E)-d\right|=\mathcal{O}\left(n^{0}\right)$, and end points, $n-d, d=\mathcal{O}\left(n^{0}\right)$. In Fig. 4 , we plot the comparison between the coefficients $c(E, d)$ computed based on the exact expression (23) and the results of asymptotic WKB analysis using Eqs. (B10) and (B13).

\section{APPENDIX C: LINEARIZATION OF THE DOWN-FOLDED HAMILTONIAN NEAR THE CENTER OF THE IMPURITY BAND}

We divide the Hamiltonian $\mathcal{H}(E)$ for a given $E$ on two parts, accordingly:

$$
\mathcal{H}_{i j}(E)=\mathcal{H}_{i j}^{(0)}(E)+\mathcal{H}_{i j}^{(1)}(E),
$$

where we define

$$
\mathcal{H}_{i j}^{(0)}(E)=n[c(E, 0)-1] \delta_{i j}
$$

$\mathcal{H}_{i j}^{(1)}(E)=\delta_{i j}[1-c(E, 0)] \epsilon_{i}+n c\left(E, d_{i j}\right)\left(1-\delta_{i j}\right)$.

We write similar expansions for energies and amplitudes:

$E \approx E^{(0)}+E^{(1)}, \quad \psi\left(z_{j}\right) \approx \psi^{(0)}\left(z_{j}\right)+\psi^{(1)}\left(z_{j}\right)$,

and get

$$
\mathcal{H}(E) \approx \mathcal{H}^{(0)}\left(E^{(0)}\right)+\frac{\partial \mathcal{H}^{(0)}\left(E^{(0)}\right)}{\partial E} E^{(1)}+\mathcal{H}^{(1)}\left(E^{(0)}\right),
$$

where the parts of the Hamiltonian $\mathcal{H}^{(0,1)}$ are given above. We plug the above expansions into the system of equations (17) $\sum_{j=1}^{M} \mathcal{H}_{i j}(E) \mathcal{A}_{j}=E \mathcal{A}_{i}$ and use Eq. (A13) to express $\mathcal{A}_{j}^{(0)}=n^{1 / 2} \psi^{(0)}\left(z_{j}\right)$. Equating terms of the same order in $\epsilon_{j}$ and $c\left(E, d_{i j}\right), i \neq j$, we obtain the equation for eigenstates and eigenvalues in the zeroth order:

$$
n\left[c\left(E^{(0)}, 0\right)-1\right] \psi^{(0)}\left(z_{j}\right)=E^{(0)} \psi^{(0)}\left(z_{j}\right),
$$

$j \in[1 \ldots M]$, and in the first order:

$$
\begin{aligned}
& a \epsilon_{i} \psi^{(0)}\left(z_{j}\right)+b \sum_{j \neq i=1}^{M} n c\left(E^{(0)}, d_{i j}\right) \psi^{(0)}\left(z_{j}\right) \\
& =E^{(1)} \psi^{(0)}\left(z_{j}\right) .
\end{aligned}
$$

The above index $j$ enumerates marked states. Also, the coefficients $a$ and $b$ equal

$a=b\left[1-c\left(E^{(0)}, 0\right)\right], \quad b^{-1}=1-n \frac{\partial c\left(E^{(0)}, 0\right)}{\partial E}$.

Similarly to the above, we find from Eqs. (21) and (22) the zeroth-order approximation to the total probabilistic weight of an eigenfunction $|\psi\rangle$ over the marked state subspace $Q_{j k}^{(0)}=\delta_{j k} Q$, where

$Q_{j k}^{(0)}=\delta_{j k} Q, \quad \frac{1}{Q}=n^{2} \frac{\partial}{\partial E}\left(\frac{c(E, 0)-1}{E}\right)_{E=E^{(0)}}$.

\section{Zeroth order of the perturbation theory}

Equation (C5) admits the solution corresponding to the $M$-fold degenerate energy level that originates from the band of the marked states, $E^{(0)} \rightarrow-n$ in the limit of $B_{\perp} \rightarrow 0$. The corresponding $M$ eigenstates $\psi_{\beta}\left(z_{j}\right)$ $(\beta \in[1 \ldots M])$ have support over the part of computational basis corresponding to marked states: $\psi_{z_{j}}^{\beta} \neq 0, j \in(1, M)$. Using $c(E, 0)$ from Eq. (C16), the explicit form of Eq. (C5) 
for the eigenvalue in the zeroth order is given in the main text [Eqs. (36) and (37)], which we repeat here for convenience:

$$
\begin{gathered}
E^{(0)}=-n-\Delta_{0}, \\
\Delta_{0}=n 2^{-n} \sum_{d=0}^{n}\left(\begin{array}{l}
n \\
d
\end{array}\right) \frac{B_{\perp}(n-2 d)}{n+\Delta_{0}-B_{\perp}(n-2 d)} .
\end{gathered}
$$

Here, $\Delta_{0}$ is the root of the above transcendental equation that satisfies the condition $\lim _{B_{\perp} \rightarrow 0} \Delta_{0}=0$. In general, the sum (37) is dominated by the region of values of $d$ such that $|d-n / 2|=\mathcal{O}\left(n^{1 / 2}\right)$. We obtain $\Delta_{0}$ in the form of a series expansion in powers of $n^{-1}$ :

$$
\Delta_{0} \simeq-B_{\perp}^{2}-\frac{B_{\perp}^{4}}{n}+\mathcal{O}\left(n^{-2}\right) .
$$

Similarly, using $c(E, 0)$ from Eq. (23) in Eq. (C8) for the zeroth-order total weight over the marked state subspace, we obtain

$$
\begin{gathered}
\sum_{k=1}^{M}\left|\psi_{z_{k}}^{(0)}\right|^{2}=Q, \\
\frac{1}{Q}=\frac{1}{2^{n}} \sum_{d=0}^{n}\left(\begin{array}{l}
n \\
d
\end{array}\right) \frac{1}{\left[B_{\perp}(n-2 d)-n-\Delta_{0}\right]^{2}} .
\end{gathered}
$$

Using Eq. (38) and employing similar approximations to that from the above, we get an asymptotical expression in the large $n$ limit:

$$
Q \simeq 1-\frac{B_{\perp}^{2}}{n}-\frac{3 B_{\perp}^{4}}{n^{2}}+\mathcal{O}\left(n^{-3}\right) .
$$

We recall that in our study $n$ is asymptotically large and we always assume that the transverse field $B_{\perp}=\mathcal{O}\left(n^{0}\right)$ (but can be parametrically large, $B_{\perp} \gg 1$ ).

The denominator in Eqs. (C10) and (C12) corresponding to $d=m$ becomes zero at "resonant" transverse-field value $B_{\perp}=B_{\perp m}$ where $d=m$ denominator in the right hand side of Eq. (C10) vanishes. In the range of $B_{\perp}$ under consideration, $n / 2-m \gg n^{1 / 2}$.

Near the $m$ th resonance, the term with $d=m$ in the sum (37) becomes anomalously large due to a small denominator despite the factor $p_{m}$ being very small. We keep this term (37) along with the terms corresponding to $|n / 2-d| \sim n^{1 / 2}$ and obtain

$$
\Delta_{0} \simeq \frac{\delta_{B}}{2} \pm \sqrt{\frac{\delta_{B}^{2}}{4}+n^{2} p_{m}},
$$

where we introduce a rescaled transverse-field difference from its value at resonance:

$$
\delta_{B}=n \frac{B_{\perp}-B_{\perp m}}{B_{\perp m}^{(0)}},
$$

where $B_{\perp m}^{(0)}=n /(n-2 m)$.

Clearly, in the resonance region $\delta_{B} \sim n p_{m}^{1 / 2}$ and $\left|B_{\perp}-B_{\perp m}\right| \sim \Delta B_{\perp m}$, where $\Delta B_{\perp m} \sim 2^{-n / 2}\left(\begin{array}{c}n \\ m\end{array}\right) B_{\perp m}^{(0)}$. There, the weight factor $Q$ is decreasing dramatically (cf. Fig. 7), and the above perturbation theory breaks down. The width of the resonant regions $\Delta B_{\perp m}$ remains exponentially small in $n$ for $n / 2-m \gg n^{1 / 2}$.

In this study, we focus only on the off-resonance case, assuming the condition

$$
\Delta B_{\perp m} \ll\left|B_{\perp m}-B_{\perp}\right| \sim\left|B_{\perp m+1}-B_{\perp}\right|=\mathcal{O}\left(B_{\perp}\right) .
$$

\section{First order of the perturbation theory}

The first-order equation (C6) determines the correct zeroth-order eigenstates $\left\{\psi_{\beta}\left(z_{j}\right)\right\}_{\beta=1}^{M}$ and removes the degeneracy of the energy levels. To evaluate the coefficients $a$ and $b$ in Eq. (C6), we calculate $c(E, 0)$ away from resonance using the same approach as that in the evaluation of the sum in Eq. (37):

$$
c(E, 0) \simeq-\frac{n B_{\perp}^{2}}{E^{2}}+\mathcal{O}\left(\frac{n^{2} B_{\perp}^{4}}{E^{4}}\right) .
$$

The coefficients $a, b \simeq 1+\mathcal{O}\left(B_{\perp}^{2} / n\right)$ and in what follows are replaced by unity. Then, Eq. (C6) corresponds to the effective Hamiltonian $\mathcal{H}$ with the matrix elements $\mathcal{H}_{i i}=\epsilon_{i}$ and $\mathcal{H}_{i j \neq i}=n c\left(E^{(0)}, d_{i j}\right)$, where coupling coefficients $c$ are given in Eq. (33). Using Eqs. (36) and (37) for zerothorder energy $E^{(0)}$, the matrix $\mathcal{H}_{i j}$ can be written in the form (41).

\section{APPENDIX D: STATISTICAL INDEPENDENCE OF MATRIX ELEMENTS}

In this paper, the IB Hamiltonian $\mathcal{H}_{i j}$ is determined by the symmetric matrix of Hamming distances $d_{i j}$ between the bit strings corresponding to the marked states sampled without replacement from the set of all possible $2^{n}$ bit strings. Instead of this ensemble, one can consider a different one, where each of the $M$ bit strings is sampled with replacement from the full set $\{0,1\}^{n}$. In this ensemble, Hamming distances $d_{i j}$ for distinct pairs $i, j$ are statistically independent, allowing for much simpler statistical averaging. Indeed, for a given row $i$ of the matrix $d_{i j}$, the joint 
probability distribution of the two distinct off-diagonal matrix elements can be estimated as

$$
p_{d_{i j_{1}}, d_{i j_{2}}}-p_{d_{i j_{1}}} p_{d_{i j_{2}}} \propto \frac{1}{2^{n}} \Delta\left(d_{i j_{1}}-d_{i j_{2}}\right) p_{d_{i j_{1}}} .
$$

Here, $\Delta(d)$ denotes the Kronecker delta, $j_{1} \neq j_{2} \neq i$, and $p_{d}$ as before corresponds to the modified binomial distribution:

$$
p_{d}=\frac{1}{Z} 2^{-n}\left(\begin{array}{l}
n \\
d
\end{array}\right), \quad Z=\sum_{d=1}^{n} 2^{-n}\left(\begin{array}{l}
n \\
d
\end{array}\right)
$$

(also, $\sum_{d_{1}, d_{2}=1}^{n} p_{d_{1}, d_{2}}=1$ ). One can see that the statistical correlation between a pair of Hamming distances $d_{i j_{1}}, d_{i j_{2}}$ is exponentially small (in $n$ ) and can be neglected.

Such an ensemble allows for multiple copies of the same bit string to be sampled. However, this effect is not statistically significant for modest values of $M$ :

$$
1 \ll M \ll 2^{n / 2} .
$$

This result can be seen by comparing the number of ways to perform unordered sampling of $M$ elements from the group of $2^{n}$ elements with and without replacement. Using Stirling's formula, we write the former number as

$$
\left(\begin{array}{c}
2^{n}+M-1 \\
M
\end{array}\right) \simeq\left(\begin{array}{c}
2^{n} \\
M
\end{array}\right) \exp \left(\frac{M^{2}}{2^{n}}\right)(1+\varepsilon)
$$

where the latter number is given by $\left(\begin{array}{l}2^{n} \\ M\end{array}\right)$ with $\varepsilon \sim M 2^{-3 n / 4} \ll 1$. It is clear that when condition (D3) is satisfied the two ensembles are statistically equivalent, because repetitions can be neglected.

\section{APPENDIX E: BOUND ON THE LARGEST EIGENVALUE OF $\mathcal{V}_{i j}$ FROM GERSCHGORIN CIRCLE THEOREM}

One can use the above estimates of the typical largest matrix elements of the matrix $\mathcal{V}_{i j}$ to consider the bounds on its eigenvalues given by the Gerschgorin circle theorem [58]. For the case of real eigenvalues, the theorem states that every eigenvalue lies within at least one of the intervals $\left[\mathcal{V}_{i i}-R_{i}, \mathcal{V}_{i i}+R_{i}\right]$, where $i \in[1 \ldots M]$ and $R_{i}=\sum_{j \neq i}\left|\mathcal{V}_{i j}\right|$ is a sum of absolute values of the off-diagonal elements in the $i$ th row. For a randomly chosen row, the value of $R_{i}$ can be estimated as follows:

$$
R_{i} \simeq M \sum_{d=1}^{n} p_{d}|V(d)|
$$

where $p_{d}$ is defined in Eq. (49). From Eq. (42), one can see that the above sum is dominated by the terms satisfying $|n / 2-d| \ll n$. Using Stirling's approximation, we get
$R_{i} \sim M 2^{-n / 2} e^{-n \theta}$. For typical diagonal matrix elements, $\left|\mathcal{V}_{i i}\right|=\left|\epsilon_{i}\right| \lesssim W$. Therefore, from the Gerschgorin theorem, we conclude the eigenvalues $E_{\beta}^{(1)}$ of $\mathcal{H}$ satisfy the following bound:

$$
\left|E_{\beta}^{(1)}\right| \leq \max \left\{W, M 2^{-n / 2} e^{-n \theta}\right\} .
$$

One can see that the Gerschgorin bound in our case precisely corresponds to the typical maximum element in the matrix $\mathcal{H}_{i j}$.

\section{APPENDIX F: MEAN VALUE AND STANDARD DEVIATION OF THE OFF-DIAGONAL MATRIX ELEMENTS $\mathcal{H}_{i j}$}

The mean value of the off-diagonal matrix element

$$
\left\langle\mathcal{H}_{i j}\right\rangle=n \sum_{d=0}^{n} p_{d} c(E, d) \simeq \frac{n}{2^{n}} \frac{B_{\perp}}{B_{\perp}-1}
$$

is much smaller than its standard deviation

$$
\left\langle\left(\mathcal{H}_{i j}-\left\langle\mathcal{H}_{i j}\right\rangle\right)^{2}\right\rangle^{1 / 2} \simeq B_{\perp} \sqrt{\frac{n}{2^{n}}} .
$$

This difference is related to the symmetry $p_{d}=p_{n-d}$ and a rapid oscillation of $c\left(E^{(0)}, d\right)$ with $d$ [cf. Eqs. (33) and (B11) and Fig. 4].

We note from Eqs. (51) and (F2) that the standard deviation is exponentially larger than the typical value:

$$
\left\langle\left(\mathcal{H}_{i j}-\left\langle\mathcal{H}_{i j}\right\rangle\right)^{2}\right\rangle^{1 / 2} \sim V_{\text {typ }} e^{n \theta} .
$$

This difference can be understood by looking at the values of $d_{i j}$ that dominate the variance of $\mathcal{H}_{i j}$. We write

$$
\left\langle\left(\mathcal{H}_{i j}\right)^{2}\right\rangle=n^{2} \sum_{d=0}^{n} c^{2}\left(E^{(0)}, d\right) p_{d} .
$$

It follows from Eqs. (33) and (49) that for $d \in\left(n / 2-m_{0}\right.$, $\left.n / 2+m_{0}\right)$ the coefficient $c^{2}(E, d) \propto 1 /\left(\begin{array}{l}n \\ d\end{array}\right)$ decreases exponentially with $d$, while the distribution $p_{d} \propto\left(\begin{array}{l}n \\ d\end{array}\right)$ increases exponentially with $d$. The binomial factors cancel out, and the expression under the summation in Eq. (F3) contains a very slowly varying with $d$ (nonoscillatory) part. However, for $d \in\left(0, n / 2-m_{0}\right)$, the coefficient $c(E, d)$ grows exponentially faster than $1 /\left(\begin{array}{l}n \\ d\end{array}\right)$ with decreasing $d$ [see Eqs. (B13) and (29)]. Therefore, the variance (F3) is dominated by nonextensive values of $d=\mathcal{O}\left(n^{0}\right)$ that are much smaller than the smallest Hamming distance $d_{\text {min }}=\mathcal{O}(n)$ [Eq. (52)] in a randomly chosen row of $d_{i j}$. Therefore, the variance of $\mathcal{H}_{i j}$ is not a good statistical characteristic of the PDF of $\mathcal{H}_{i j}$. It is dominated by the extremely rare atypical instances of the ensemble. 


\section{APPENDIX G: PDF OF THE SQUARED OFF-DIAGONAL MATRIX ELEMENTS OF IMPURITY BAND HAMILTONIAN}

In this section, we provide the details of the derivation of the PDF for the nonoscillatory parts of the (squared) offdiagonal matrix elements $\mathcal{V}_{i j}^{2}$ of the IB Hamiltonian. As discussed in the main text, in the asymptotical limit of large $n \gg 1$, one can make an approximation that $n$ is a continuous variable, and we replace the summation over $d$ in Eq. (50) by an integral and Kronecker delta $\delta(x)$ by a Dirac delta. This results in Eq. (55), displayed below for convenience:

$$
P\left(\mathcal{V}_{i j}^{2}\right)=\int_{0}^{n} p_{x} \delta\left[V^{2}(x)-\mathcal{V}_{i j}^{2}\right] d x
$$

It is discussed in the main text (see also below) that the condition for this validity of this approximation is

$$
\frac{1}{n} \log _{2} M \ll 1 \text {. }
$$

It corresponds to the number of marked states $M$ that is not very large. For example, it can still scale exponentially with $n$ so that $M=2^{\mu n}, \mu=\mathcal{O}\left(n^{0}\right)$, but the coefficient $\mu$ in the exponent needs to be small, $\mu \ll 1$.

The expression (G1) is obtained using the analytical continuation $p_{x}$ of the binomial distribution $p_{d}$ [Eq. (49)] from the integer domain $d \in[0, n]$ onto the interval of a real axis $x \in[0, n]$ in terms of the Beta function $B(x, y)$ :

$$
p_{x}=2^{-n}\left(\begin{array}{l}
n \\
x
\end{array}\right)=\frac{2^{-n}}{(n+1) B(x+1, n+1-x)}
$$

and the resulting identity

$$
\int_{0}^{n} d x p_{x}=1
$$

In what follows, we study the rescaled quantities

$$
w_{i j} \equiv \frac{\mathcal{V}_{i j}^{2}}{V_{\text {typ }}^{2}}=\left(\frac{2}{\pi n}\right)^{1 / 2} \frac{1}{p_{d_{i j}}},
$$

where $i \neq j, V_{\text {typ }}$ is given in Eq. (51), and $p_{d}=2^{-n}\left(\begin{array}{l}n \\ d\end{array}\right)$. Using Stirling's approximation in the binomial coefficient

$$
\begin{aligned}
& p_{x} \equiv p_{B}(x / n), \quad p_{B}(\rho)=\frac{e^{-n \mathcal{A}(\rho)}}{\sqrt{2 \pi n \rho(1-\rho)}}, \\
& \mathcal{A}(\rho)=\rho \log \rho+(1-\rho) \log (1-\rho)+\log 2,
\end{aligned}
$$

we get from Eq. (42) for $\mathcal{V}_{i j}=V\left(d_{i j}\right)$

$$
\mathbb{w}(\rho) \equiv \frac{V^{2}(n \rho)}{V_{\mathrm{typ}}^{2}} \simeq \frac{\sqrt{4 \rho(1-\rho)}}{v(\rho)} e^{n \mathcal{A}(\rho)}
$$

where $v(\rho)$ is given in Eq. (44). Equation (G5) takes the form

$$
w_{i j}=\mathbb{w}\left(d_{i j} / n\right) .
$$

Then, the expression for the PDF for $w_{i j}$,

$$
g\left(w_{i j}\right)=V_{\mathrm{typ}}^{2} P\left(V_{\mathrm{typ}}^{2} w_{i j}\right),
$$

can be written in the form [cf. Eq. (G1)]

$$
g(w)=2 n \int_{0}^{1 / 2} p_{B}(\rho) \delta[w-\mathbb{w}(\rho)] d \rho .
$$

We note that the domain of $g(w)$ is bounded from below by $w=1$ and from above by $w=\mathcal{O}\left(2^{n}\right)$. Taking the integral in Eq. (G10), we get

$$
g(w)=2 n \frac{p_{B}\left(\rho_{w}\right)}{\left|\frac{d w(\rho)}{d \rho}\right|_{\rho=\rho_{w}}},
$$

where the rescaled Hamming distance $\rho_{w}$ is a root of the transcendental equation

$$
\mathfrak{w}\left(\rho_{w}\right)=w .
$$

In the leading order in $n \gg 1$, this equation gives

$$
\mathcal{A}\left(\rho_{w}\right)=\frac{1}{n} \log w,
$$

where $\mathcal{A}(\rho)$ is given in Eq. (G6b). Also using Eqs. (G6) and (G7) in Eq. (G11), we get

$$
g(w)=\frac{1}{w^{2} \sqrt{\pi \ell(w)}},
$$

where

$$
\ell(w)=\frac{n}{8} v^{2}\left(\rho_{w}\right)\left|\log \left(\rho_{w}^{-1}-1\right)\right|^{2} .
$$

Here, the dependence of $\ell(w)$ on $w$ is shown in Fig. 17. In the entire range, the dependence is logarithmically slow.

We note that Eq. (G13) is a valid approximation to Eq. (G12) for $\rho-\rho_{0} \gg 1 / n$, where $\rho_{0}$ is a zero of $v(\rho)$ :

$$
v\left(\rho_{0}\right)=0, \quad \rho_{0}=\frac{1}{1-\sqrt{1-B_{\perp}^{-2}}} .
$$

It corresponds to Hamming distance $n \rho_{0}=n / 2-m_{0}$ [Eq. (32)], which lies at the boundary of the interval (B9) 
where the WKB solution (33) and (42) applies (see the discussion in Sec. IV). It is assumed that $n \rho_{0}$ is smaller than the typical smallest Hamming distance $d_{\min }$ in a randomly selected row:

$$
d_{\min }-n \rho_{0} \gg 1 .
$$

Using the asymptotic expression (G6) for the binomial distribution in Eqs. (52), we get the equation for $d_{\min }$ in the form

$$
\mathcal{A}\left(d_{\min } / n\right)=\frac{1}{n} \log M
$$

The function $\mathcal{A}(\rho)$ is decreasing with $\rho$ for $\rho(0,1 / 2)$. Therefore, Eq. (G17) leads to the condition $\mathcal{A}\left(\rho_{0}\right)-$ $\mathcal{A}\left(d_{\min } / n\right) \gg 1 / n$, or

$$
\mathcal{A}\left(\rho_{0}\right)-\frac{1}{n} \log M \gg \frac{1}{n} .
$$

Using explicit forms of $\mathcal{A}(\rho)$ and $\rho_{0}$, we get in the limit of $B_{\perp} \gg 1$

$$
\begin{aligned}
\log 2-\frac{1}{n} \log M & >\frac{2 \log B_{\perp}+2 \log 2+1}{4 B_{\perp}^{2}}+\varepsilon \\
0 & <\varepsilon=\mathcal{O}\left(B_{\perp}^{-4}\right) .
\end{aligned}
$$

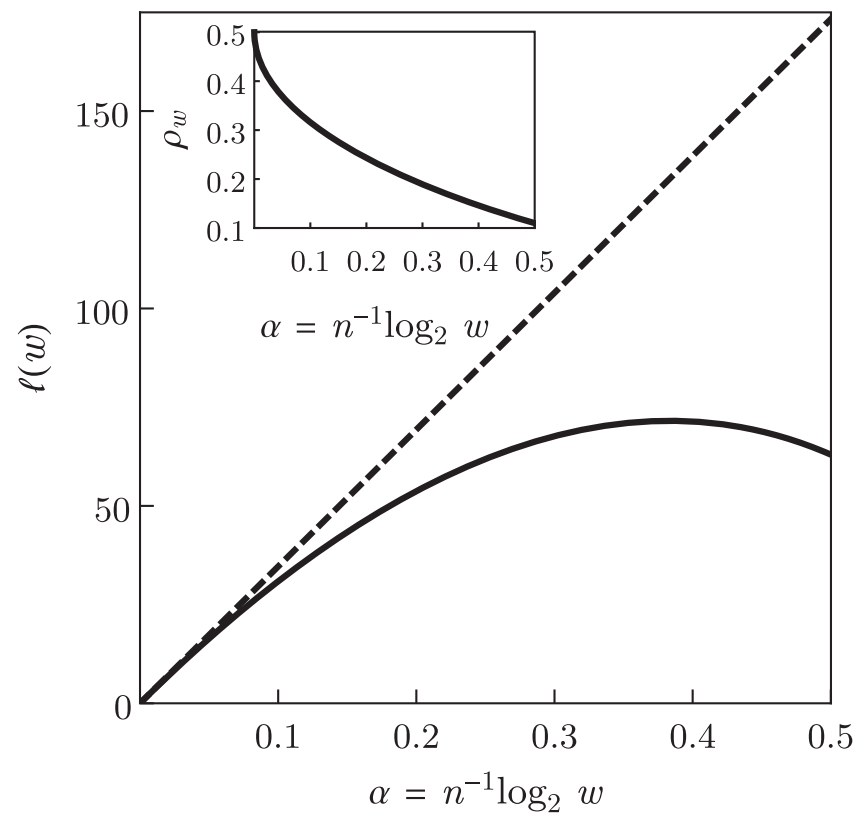

FIG. 17. The solid line shows the dependence of $\ell(w)$ on $\alpha=$ $(1 / n) \log _{2} w$ from Eq. (G15). The dashed line shows the tangent to the solid curve at the point $\alpha=0(w=1)$. This line corresponds to $\ell(w) \simeq \sqrt{\log w}$, in accordance with Eq. (G24). The inset shows the dependence of the root $\rho_{w}$ of Eq. (G12) on $\alpha=\log _{2} w^{1 / n}$. Small $\alpha \ll 1$ corresponds to Hamming distances $\rho_{w} \approx 1 / 2$. Near that point, the dependence of $\rho_{w}$ on $\alpha$ follows Eq. (G23).
This equation is the condition for Eq. (G17). Clearly, it corresponds to a much weaker constraint on the values of $M$ than the condition $(1 / n) \log M<\frac{1}{2} \log 2$ provided by the requirement of a statistical independence of matrix elements of $\mathcal{V}_{i j}$ [cf. Eq. (D3)].

\section{Case of $(1 / n) \log _{2} M \ll 1$}

The rescaled Hamming distance $\rho_{w}$ depends on $w$ via the logarithmic factor $\alpha=(1 / n) \log _{2} w$. This dependence is shown in the inset in Fig. 17. In this section, we consider

$$
\alpha=\frac{1}{n} \log _{2} w \ll 1 .
$$

Then, we get

$$
\begin{aligned}
p_{B}(\rho) & \simeq\left(\frac{2}{\pi n}\right)^{1 / 2} e^{-n \mathcal{A}(\rho)}, \\
\mathcal{A}(\rho) & \simeq 2\left(\frac{1}{2}-\rho\right)^{2} .
\end{aligned}
$$

Then, using Eq. (G13), we get

$$
\begin{gathered}
\rho_{w} \simeq \frac{1}{2}-\left(\frac{\alpha}{2}\right)^{1 / 2}, \\
\ell(w) \simeq \log w,
\end{gathered}
$$

and, finally,

$$
g(w) \simeq g_{\infty}(w)=\frac{1}{w^{2} \sqrt{\pi \log w}} .
$$

The subscript here indicates that, unlike $g(w)$, the PDF $g_{\infty}(w)$ has the upper boundary of its domain equal to infinity. It is of interest to calculate for a given $w$ the magnitude of the relative changes of $V^{2}(d)$ and of the binomial coefficient $p_{d}$ when the Hamming distance is chaining by 1 (and $\rho_{w}$ is changing by $1 / n$ ). We define as in Eqs. (G8) and (G7) $w=V^{2}(d) / V_{\text {typ }}^{2}$ and obtain

$$
\begin{gathered}
\frac{V^{2}(d+1)-V^{2}(d)}{V^{2}(d)} \simeq \frac{p_{d+1}-p_{d}}{p_{d}} \\
\simeq 4\left(\frac{1}{2}-\rho_{w}\right)=\sqrt{8 \alpha} \ll 1 .
\end{gathered}
$$

Here, we use Eqs. (G23) and (G21). The above inequality justifies using the continues approximation (G1) in Eq. (50).

In a randomly chosen row of $w_{i j}$, the PDF that the largest element equals $w$ is 
$\operatorname{PDF}\left(\max _{m} w_{m}=w\right) \simeq \frac{M e^{-M w \log w}}{w \log w}, \quad M \gg 1$.

The typical largest element in a row $\max _{1<j<i} w_{i j} \sim M$ in agreement with the results obtained earlier; cf. Eqs. (42), (52), and (G5). Therefore, in order to ensure that $\alpha \ll 1$ for all matrix elements in a typical row of $w_{i j}$, we require that $\log _{2} M \ll n$ :

$$
1 \leq w \lesssim M, \quad \frac{1}{n} \log _{2} M \ll 1
$$

The typical value of the smallest element in a randomly selected row of the rescaled matrix of Hamming distances $d_{i j} / n$ equals

$$
\rho_{\min }=\frac{d_{\min }}{n}=\frac{1}{2}-\sqrt{\frac{\log _{2} M}{2 n}} .
$$

We note that in the case we consider,

$$
n \gg n / 2-d_{\min }=\mathcal{O}(n),
$$

minimum value $d_{\min }$ is close to $n / 2$ but is still separated by an extensive distance from it.

In this paper, we use the expression for the matrix elements of the IB Hamiltonian $\mathcal{H}_{i j}$ (41) that applies only in the region $\left|n / 2-d_{i j}\right|<m_{0}$, where $m_{0}$ is given in Eq. (32). The elements in a typical row of the matrix $d_{i j}$ belong to this region if the condition $\left|n / 2-d_{\min }\right|<m_{0}$ is fulfilled. Using Eq. (G30), we can rewrite this expression as an inequality for $M$ :

$$
M<2^{\frac{n}{2}\left(1-B_{\perp}^{-2}\right)} .
$$

This inequality is satisfied under the condition (G29).

\section{APPENDIX H: CHARACTERISTIC FUNCTION OF THE PDF OF THE SQUARED OFF-DIAGONAL MATRIX ELEMENTS OF IMPURITY BAND HAMILTONIAN}

Here, we compute the characteristic function of the PDF $g_{\infty}(w)$ [Eq. (G25)] [also given in Eq. (60) of the main text]. It is defined as follows:

$$
\phi_{\infty}(u)=\int_{1}^{\infty} d w g_{\infty}(w)\left(e^{i u w}-1\right) .
$$

We are interested in the asymptotic limit of the above expression at small $|u| \ll 1$. It is convenient to calculate separately the real and imaginary parts of $\phi_{\infty}(u)$.
For the real part, we have

$$
-\frac{\sqrt{\pi}}{2} \operatorname{Re}\left[\phi_{\infty}(u)\right]=\int_{1}^{\infty} \frac{1}{x^{2} \sqrt{\log x}} \sin ^{2}\left(\frac{u x}{2}\right) .
$$

Because $\phi_{\infty}(-u)=\phi_{\infty}^{*}(u)$, we can assume that $u>0$ and break the interval of integration above in two parts:

$$
x \in[1, X / u] \cup[X / u, \infty), \quad u \ll X \ll 1 .
$$

We write

$$
-\frac{\sqrt{\pi}}{2} \operatorname{Re}\left[\phi_{\infty}(u)\right]=R_{1}(u)+R_{2}(u) .
$$

Here,

$$
\begin{aligned}
R_{1}(u) & =\int_{1}^{X / u} \frac{1}{x^{2} \sqrt{\log x}} \sin ^{2}\left(\frac{u x}{2}\right), \\
R_{2}(u) & =\int_{X / u}^{\infty} \frac{1}{x^{2} \sqrt{\log x}} \sin ^{2}\left(\frac{u x}{2}\right) .
\end{aligned}
$$

Using Eq. (H3), the asymptotic expansion of $R_{1}(u)$ has the form

$$
R_{1}(u) \simeq \frac{u X}{4[\log (1 / u)]^{1 / 2}}+\frac{u X \log (1 / X)}{8[\log (1 / u)]^{3 / 2}}+\cdots
$$

Also, after some tedious calculations, we obtain

$$
\begin{aligned}
R_{2}(u) \simeq & \frac{u}{[\log (1 / u)]^{1 / 2}}\left(\frac{\pi}{4}-\frac{X}{4}\right) \\
& +\frac{u}{2[\log (1 / u)]^{3 / 2}} \frac{\pi\left(\gamma_{\text {Euler }}-1\right)}{4}
\end{aligned}
$$

where

$$
\gamma_{\text {Euler }} \simeq 0.577
$$

is the Euler constant.

Similarly to the above, we also break the interval of integration in the imaginary part of $\phi_{\infty}(u)$ in two parts given in Eq. (H3):

$$
\operatorname{Im}\left[\phi_{\infty}(u)\right]=I_{1}(u)+I_{2}(u),
$$

where

$$
\begin{aligned}
& I_{1}(u)=\int_{1}^{X / u} \frac{\sin u x}{x^{2} \sqrt{\pi \log x}}, \\
& I_{2}(u)=\int_{X / u}^{\infty} \frac{\sin u x}{x^{2} \sqrt{\pi \log x}} .
\end{aligned}
$$


Expanding the integrand (H11) in $u$ and using condition (H3), we get

$$
I_{1}(u) \simeq \frac{2 u \sqrt{\log \frac{1}{|u|}}}{\sqrt{\pi}}-\frac{u \log \frac{1}{X}}{\sqrt{\pi \log \frac{1}{|u|}}}+\mathcal{O}\left(\frac{u \log ^{2} X}{\log ^{3 / 2}|u|}\right) .
$$

Performing a similar asymptotic expansion in $I_{2}(u)$, we obtain

$$
I_{2}(u) \simeq \frac{u\left(1-\gamma_{\text {Euler }}-\log X\right)}{\sqrt{\pi \log \frac{1}{|u|}}}+\mathcal{O}\left(\frac{u \log ^{2} X}{\log ^{3 / 2} u}\right) .
$$

Finally, we combine together Eqs. (H7) and (H8) into Eq. (H4) to obtain the first two terms in the asymptotic expansion of $\operatorname{Re}\left[\phi_{\infty}(u)\right]$ in powers of $1 / \log u \ll 1$ :

$\operatorname{Re}\left[\phi_{\infty}(u)\right]=-\frac{|u| \sqrt{\pi}}{2 \sqrt{\log |u|^{-1}}}\left(1-\frac{1-\gamma_{\text {Euler }}}{2 \log |u|^{-1}}\right)$.

We also combine together the above expressions for $I_{1}$ and $I_{2}$ to obtain a similar asymptotic expansion of $\operatorname{Im}\left[\phi_{\infty}(u)\right]$ :

$$
\operatorname{Im}\left[\phi_{\infty}(u)\right] \simeq \frac{2 u \sqrt{\log \frac{1}{|u|}}}{\sqrt{\pi}}+\frac{u\left(1-\gamma_{\text {Euler }}\right)}{\sqrt{\pi \log \frac{1}{|u|}}} .
$$

Note that in both cases the terms involving $X$ cancel out, confirming the validity of the matching procedures.

\section{APPENDIX I: GENERALIZED CENTRAL LIMIT THEOREM FOR THE SUM OF $M$ RANDOM VARIABLES $w_{m}$ THAT OBEY THE DISTRIBUTION $g_{\infty}(w)$}

In this section, we study the asymptotic PDF for the sum of the independent identically distributed (IID) random variables in Eq. (93) sampled from the probability distribution $(\mathrm{G} 25)$. We note that the variance of the random variables does not exist. The PDFs with polynomial tails at infinity are known as Pareto (heavy-tailed) distributions. According to the GCLT, the PDF of the sum of $M$ Pareto variables for $M \rightarrow \infty$ approaches its asymptotic form given by the stable law [53]. This general property coincides with the usual central limit theorem for the case when random variables in a sum have finite variances. In this case, the limiting PDF has a Gaussian form.

We note that the PDF given by Eq. (G25) is not strictly polynomial at $w \rightarrow \infty$ because of the additional logarithmic factor. We derive the asymptotic form of the sum (93) of random variables [Eq. (G25)] explicitly and compare with the standard GCLT result without the logarithmic factor.

We are interested in the PDF of the random variable $s_{M}$ such that [cf. Eqs. (60) and (93)]

$$
s_{M}=\frac{1}{M} \sum_{i=1}^{M} w_{i}, \quad g_{\infty}(w)=\frac{1}{w^{2} \sqrt{\pi \log w}} .
$$

Here, $w_{i}$ are IID random variables sampled from $g_{\infty}(w)$, and we are interested in the asymptotic limit $M \gg 1$.

Using the convolution property of a sum of statistically independent random variables, we get for the PDF of $s_{M}$

$$
\operatorname{PDF}\left(s_{M}\right)=\frac{1}{2 \pi} \int_{-\infty}^{\infty} d q\left[\varphi_{\infty}(q / M)\right]^{M} e^{-i q s_{M}},
$$

where

$$
\varphi_{\infty}(u)=1+\phi_{\infty}(u)
$$

and $\phi_{\infty}(u)$ is given in Eq. (H1). The limit $M \gg 1$ corresponds to $|u| \ll 1$. We note that

$$
\lim _{u \rightarrow 0} \phi_{\infty}(u)=0 .
$$

Taking into account that $\phi(u)$ is small in the above limit, we write

$\operatorname{PDF}\left(s_{M}\right) \simeq \frac{1}{2 \pi} \int_{-\infty}^{\infty} d q \exp \left[-i q s_{M}+M \phi_{\infty}(q / M)\right]$.

The quantity $M \phi_{\infty}(q / M)$ can be expanded in inverse powers of $\log M \gg 1$ using the asymptotic form of the characteristic function at a small argument given in Eqs. (H13) and (H14). The first few terms of expansion have the form

$$
\begin{aligned}
& M \operatorname{Re} \phi_{\infty}\left(\frac{q}{M}\right) \simeq-\frac{\pi|q|}{2 \sqrt{\log M}}+\frac{\sqrt{\pi}|q|\left(1-\gamma_{\text {Euler }}-\log |q|\right)}{4(\log M)^{3 / 2}}, \\
& M \operatorname{Im} \phi_{\infty}\left(\frac{q}{M}\right) \simeq 2 q\left(\frac{\log M}{\pi}\right)^{1 / 2}+q \frac{1-\gamma_{\text {Euler }}-\log |q|}{(\pi \log M)^{1 / 2}} \\
& +\frac{q \log |q|\left(1-\gamma_{\text {Euler }}\right)}{2 \sqrt{\pi}(\log M)^{3 / 2}},
\end{aligned}
$$

where $\gamma_{\text {Euler }}$ is the Euler constant.

It is clear from comparing individual terms in Eqs. (I5) with the exponential in the integrand in Eq. (I4) that $q=\mathcal{O}(\sqrt{\log M})$. Therefore, we can drop in Eqs. (I5) terms $\mathcal{O}\left[1 /(\log M)^{3 / 2}\right]$. We make the change of variables in the integral in Eq. (I4)

$$
q=2 \sqrt{\frac{\log M}{\pi}} t
$$

and obtain

$$
\operatorname{PDF}\left(s_{M}\right)=\frac{1}{\sigma_{M}} L_{1}^{1,1}\left(\frac{s_{M}-b_{M}}{\sigma_{M}}\right),
$$




$$
L_{1}^{1,1}(x) \equiv \frac{1}{2 \pi} \int_{-\infty}^{\infty} d t e^{-i t x-|t|-[(2 i) / \pi] t \log |t|}
$$

The function $L_{1}^{1,1}(x)$ above is a so-called Levy alpha-stable distribution $[33,61,62]$ shown in Fig. 15. The distribution is defined by its characteristic function. Parameters $b_{M}$ and $\sigma_{M}$ in Eq. (I7) are typical values that characterize the shift of the maximum of the $\operatorname{PDF}\left(s_{M}\right)$ from the origin and its overall scale, respectively. They are given in Eqs. (96) and (95) of the main text, and we also provide them for convenience below:

$$
\begin{gathered}
\sigma_{M}=\frac{\pi}{2} \frac{1}{(\pi \log M)^{1 / 2}}, \\
b_{M} \simeq \sigma_{M}^{-1}-\frac{2}{\pi} \sigma_{M} \log \left(\sigma_{M}^{-1}\right)+\frac{2}{\pi}\left(1-\gamma_{\text {Euler }}\right) \sigma_{M},
\end{gathered}
$$

where $\gamma_{\text {Euler }}$ is the Euler constant.

It is instructive to compare the above expressions with the result for the sum of random variables that obey a standard Pareto distribution [i.e., without the logarithmic factor present in $\left.g_{\infty}(w)\right]$ :

$$
s_{M}^{0}=\frac{1}{M} \sum_{i=1}^{M} w_{i}, \quad w_{i} \sim g_{0}(w)=w^{-2} .
$$

The PDF of $s_{M}^{0}$ has the same form as the PDF of $s_{M}$ given in Eq. (I7), but the expressions for the shift $b_{M}^{0}$ and the overall scale $\sigma_{M}^{0}$ are different:

$$
\sigma_{M}^{0}=\frac{\pi}{2}, \quad b_{M}^{0}=\log M+1-\gamma_{\text {Euler }}+\log \left(\frac{\pi}{2}\right) .
$$

One can see that

$$
\frac{\sigma_{M}^{0}}{\sigma_{M}} \sim \frac{b_{M}^{0}}{b_{M}} \sim(\log M)^{1 / 2} \gg 1 .
$$

The rescaling factor $(\log M)^{1 / 2}$ between the PDFs of $s_{M}$ and $s_{M}^{0}$ can be explained by a similar logarithmic factor in the ratio $g_{0}(w) / g_{\infty}(w) \sim(\log w)^{1 / 2}$, taking into account the fact that typically $w \sim M$.

\section{APPENDIX J: JUSTIFICATION OF REPLACING SUM WITH INTEGRAL IN EQ. (84)}

We note that the number of marked states $\Omega_{d}$ in a miniband [Eq. (98)] on a Hamming distance $d$ from a given marked state $\left|z_{j}\right\rangle$ decreases rapidly when $d$. There is a typical minimum Hamming distance $d \simeq d_{\min }^{\text {res }}$ such that

$$
d_{\mathrm{min}}^{\mathrm{res}}=\operatorname{argmin}\left(\Omega_{d}\right)=\mathcal{O}(1)
$$

There will be no states in the miniband located at the Hamming distances $d$ from the state $\left|z_{j}\right\rangle$ that lie inside the intervals $d \in\left[1, d_{\mathrm{min}}^{\mathrm{res}}\right) \cup\left(n-d_{\mathrm{min}}^{\mathrm{res}}, n\right]$. For those values of $d$, we have $\Gamma_{j}^{(d)}=0$. Using Eq. (G22), we get

$$
d_{\mathrm{min}}^{\mathrm{res}} \simeq \frac{n}{2}-\sqrt{\frac{n}{2} \log \frac{2 A \Omega}{\pi n^{2}}},
$$

where $A=A\left(E^{(0)}, 1 / 2\right)$ [Eq. (43)].

On the one hand, we assume throughout this paper that the number of marked states in a miniband $\Omega \gg 1$ is sufficiently large so that the number $n-2 d_{\min }^{\text {res }}$ of dominant terms in the sum (83) is much bigger than 1 . For example, using the scaling ansatz (75), we have $\Omega \sim$ $M^{2-\gamma}$ [Eq. (100)]. Then, assuming that $\gamma<2$ and $1>(1 / n) \log _{2} M=\mathcal{O}\left(n^{0}\right)$, we can see that the second term in the rhs of Eq. (J2) is of the order of $n$ and, therefore, the number $n-2 d_{\min }^{\text {res }}=\mathcal{O}(n)$.

On the other hand, we note that the number $\Omega_{d}$ [Eq. (98)] of marked states in a miniband a Hamming distance $d$ from a given marked state $\left|z_{j}\right\rangle$ is large $\left(M_{j}^{(d)}>\Omega_{d} \gg 1\right)$ for almost all $d$, aside from $\mathcal{O}\left(n^{0}\right)$ values of $d$ near the boundaries of the interval $d \in\left[d_{\min }^{\text {res }}, n-d_{\min }^{\text {res }}\right]$.

We recall that all terms in sum (83) are nearly equal to each other, and, therefore, the relative contribution to $\Gamma_{j}$ from the boundary terms is $\mathcal{O}(1 / n)$ and can be neglected in leading-order estimates of the typical quantities. For $d$ away from the interval boundaries, the function $\delta_{\eta}\left(\epsilon_{j}-\epsilon_{m}\right)$ in Eq. (84) changes little between the adjacent values of $\epsilon_{m}$ (by an amount of the order of $1 / \Omega_{d} \ll 1$ ). This result provides the justification for us to replace the sum over $m$ in (84) by an integral.

\section{APPENDIX K: PDF OF THE RANDOM VARIABLE $\boldsymbol{h}=\left\{\boldsymbol{\eta} /\left[(z-\epsilon)^{2}+\boldsymbol{\eta}^{2}\right]\right\}$}

Consider the $\operatorname{PDF} p_{\eta}(h ; z)$ introduced in Eq. (104):

$$
p_{\eta}(h ; z)=\int_{-\infty}^{\infty} \frac{1}{W} p_{A}(\epsilon / W) \boldsymbol{\delta}[h-\delta(z-\epsilon, \eta)] d \epsilon .
$$

Here, the function of two arguments $\delta(x, y)$ is defined in Eq. (82), and $\boldsymbol{\delta}[x]$ is the Dirac delta function denoted here with bold to distinguish from the above function. We also use the relation (47) for the PDF of marked state energies. Solving

$$
h=\frac{\eta}{(z-\epsilon)^{2}+\eta^{2}}
$$

for $\epsilon$ we get

$$
\epsilon_{ \pm}=z \pm \sqrt{\eta\left(h^{-1}-\eta\right)} .
$$


From here and from Eq. (K1), we get

$$
\begin{gathered}
p_{\eta}(h ; z)=\frac{\sqrt{\eta}}{2 h^{3 / 2} \sqrt{1-\eta h}}\left[\varphi_{+}(h ; z)+\varphi_{-}(h ; z)\right], \\
\varphi_{ \pm}(h ; z)=W^{-1} p_{A}[z \pm \sqrt{\eta(1 / h-\eta)}] .
\end{gathered}
$$

For $|z| \ll W$, we get $p_{\eta}(h ; \eta) \simeq p_{\eta}(h ; 0)$ :

$$
p_{\eta}(h ; 0)=\frac{\sqrt{\eta}}{h^{3 / 2} \sqrt{1-\eta h}} p_{A}[\sqrt{\eta(1 / h-\eta)}] .
$$

\section{Uniform distribution}

For the case of uniform distribution

$$
p_{A}(\epsilon)=\frac{1}{W} \theta(W / 2-\epsilon),
$$

where $\theta(x)$ is the Heaviside theta function, we have

$$
p_{\eta}(h ; 0)=\frac{1}{h^{3 / 2} \sqrt{\eta^{-1}-h}} .
$$

The domain of values of $h$ is $h \in\left[h_{\min }, h_{\text {max }}\right]$, where

$$
\begin{gathered}
h_{\min }=\frac{1}{\eta\left(1+K_{\eta}^{2}\right)}, \quad h_{\max }=\frac{1}{\eta}, \\
K_{\eta} \equiv \frac{W}{2 \eta},
\end{gathered}
$$

and the value of the PDF on the lower boundary is

$$
p_{\eta}\left(h_{\min }\right)=\eta^{2} \frac{\left(1+K_{\eta}^{2}\right)^{2}}{K_{\eta}} .
$$

In the case of delocalized nonergodic states [Eq. (101)],

$$
M \gg K_{\eta} \gg 1 .
$$

The PDF $p_{\eta}(h ; 0) \equiv p_{\eta}(h)$ is plotted in Fig. 18. The PDF reaches the local maximum on the lower boundary $h_{\min }$ corresponding to values of marked state energies $\epsilon \simeq W$ located at the edges of the IB. In the region $h \sim 1 / \eta$, the probability density reaches very small values, $p_{\eta}(h, z) \sim \eta^{2}$, corresponding to the energies of marked states $|\epsilon-z| \simeq \eta$. The maximum value of $h=1 / \eta$ corresponds to exact resonance $\epsilon=z$. The $\operatorname{PDF} p_{\eta}(h ; 0)$ has an integrable singularity at this point.

It is of interest to consider the PDF of the sum of random variables $h_{m}$ over all marked states:

$$
s_{M}^{h}=\frac{1}{M} \sum_{m=1}^{M} h_{m}, \quad h_{m}=\frac{\eta}{\left(z-\epsilon_{m}\right)^{2}+\eta^{2}} .
$$

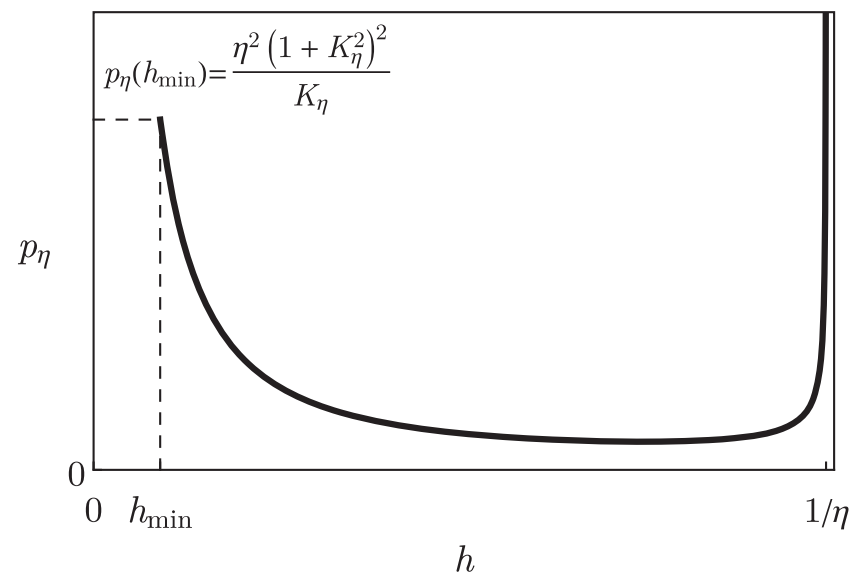

FIG. 18. Plot of the PDF of $p_{\eta}(h ; 0) \equiv p_{\eta}(h)$ given in Eq. (K6).

In the nonergodic phase $W \gg \eta$, the mean value of $h_{m}$ is much smaller than its standard deviation:

$$
\begin{gathered}
\left\langle h_{m}\right\rangle=\frac{\sum_{\sigma= \pm 1} \operatorname{arccot}\left(\frac{2 \eta}{W-2 \sigma z}\right)}{W} \simeq \frac{\pi}{W}, \\
\left\langle h_{m}^{2}\right\rangle \simeq \frac{\pi}{2 W \eta} \gg\left\langle h_{m}\right\rangle^{2} .
\end{gathered}
$$

Note that the mean is dominated by small marked state energies $\epsilon_{m} \sim \eta$, while the standard deviation is dominated by $\epsilon_{m} \sim W$.

However, for sufficiently large $M$, the mean value of the sum $\sum_{m=1}^{M} h_{m}$ is much greater than its standard deviation provided that $\delta \epsilon \ll \eta$ :

$$
\frac{\left\langle s_{M}^{h}\right\rangle^{2}-\left\langle s_{M}^{h}\right\rangle^{2}}{\left\langle s_{M}^{h}\right\rangle^{2}} \simeq \frac{1}{2 \pi} \frac{\delta \epsilon}{\eta} \ll 1 .
$$

Therefore, in the delocalized phase

$$
\eta \gg \delta \epsilon=\frac{W}{M},
$$

the sum $\sum_{m=1}^{M} h_{m}$ is self-averaging.

It is convenient to introduce rescaled variables

$$
y_{m}=\sqrt{h_{m} \eta}
$$

Their PDF has the form

$$
\mathfrak{p}_{\eta}(y)=\frac{1}{K_{\eta} y^{2} \sqrt{1-y^{2}}} .
$$

Boundaries of the domain of $\mathfrak{p}_{\eta}(y)$ are

$$
y_{\min }=\frac{1}{\sqrt{1+K_{\eta}^{2}}} \leq y<y_{\max }=1 .
$$




\section{APPENDIX L: PDF OF THE IMAGINARY PART OF SELF-ENERGY IN SELF-CONSISTENT BORN APPROXIMATION}

In this section, we provide details of calculations of the self-consistent Born approximation presented in Sec. XI A 2 of the main text. We study the PDF of the sum

$$
\Sigma^{\prime \prime}=V_{\mathrm{typ}}^{2} \sum_{m=1}^{M} \frac{w_{m} \eta}{\left(z-\epsilon_{m}\right)^{2}+\eta^{2}},
$$

where $w_{m}=\mathcal{V}^{2}\left(d_{0 m}\right) / V_{\text {typ }}^{2}$ [see Eqs. (G7) and (G8)] are random variables sampled from the distribution $g_{\infty}(w)$ [Eq. (60)] and marked state energies $\epsilon_{m}$ obey the distribution $p_{A}(\epsilon / W) / W$ [Eq. (47)]. The sum in Eq. (L1) can be written in the form

$$
\Sigma^{\prime \prime}=\frac{V_{\mathrm{typ}}^{2}}{\eta} \sum_{m=1}^{M} x_{m}, \quad x_{m}=w_{m} y_{m}^{2},
$$

where $y_{m}$ are random variables [Eq. (K18)] sampled from the distribution $\mathfrak{p}_{\eta}(y)$ [Eq. (K19)]. For $|z| \ll W$, random variables $x_{m}$ obey the PDF $g_{\eta}(x)$ such that

$$
g_{\eta}(x)=\int_{y_{\min }}^{1} d y \int_{1}^{\infty} d w \mathfrak{p}_{\eta}(y) g_{\infty}(w) \delta\left(x-w y^{2}\right)
$$

Using Eqs. (K19) and (K20), one can show that [cf. also Eq. (L7)]

$$
\lim _{\eta \rightarrow \infty} g_{\eta}(x)=g_{\infty}(x)
$$

In order to calculate the PDF of the sum $\Sigma^{\prime \prime}$ [Eq. (L1)] in the limit $M \rightarrow \infty$, we use the GCLT following the same approach as that in Appendix I. The PDF of the random variable $\Sigma^{\prime \prime}$ equals

$$
\operatorname{PDF}\left(\Sigma^{\prime \prime}\right) \simeq \frac{1}{2 \pi} \int_{-\infty}^{\infty} d k e^{-i k \Sigma^{\prime \prime}+M \phi_{\eta}\left(k V_{\mathrm{typ}}^{2} / \eta\right)}
$$

where $\phi_{\eta}(u)$ is the characteristic function of the PDF $g_{\eta}(x)$ [Eq. (L7)]:

$$
\phi_{\eta}(u)=\int_{\left[1 /\left(1+K_{\eta}^{2}\right)\right]}^{\infty} d x g_{\eta}(x)\left(e^{i u x}-1\right) .
$$

\section{PDF of individual terms in the sum}

After some transformations, we get from Eq. (L3)

$$
\begin{aligned}
g_{\eta}(x)= & \frac{1}{x^{2} K_{\eta} \sqrt{2 \pi}} \\
& \times \int_{1 / \sqrt{1+K_{\eta}^{2}}}^{\min (1, \sqrt{x})} \frac{d y}{\sqrt{\left(1-y^{2}\right)\left(\log x^{1 / 2}-\log y\right)}} .
\end{aligned}
$$

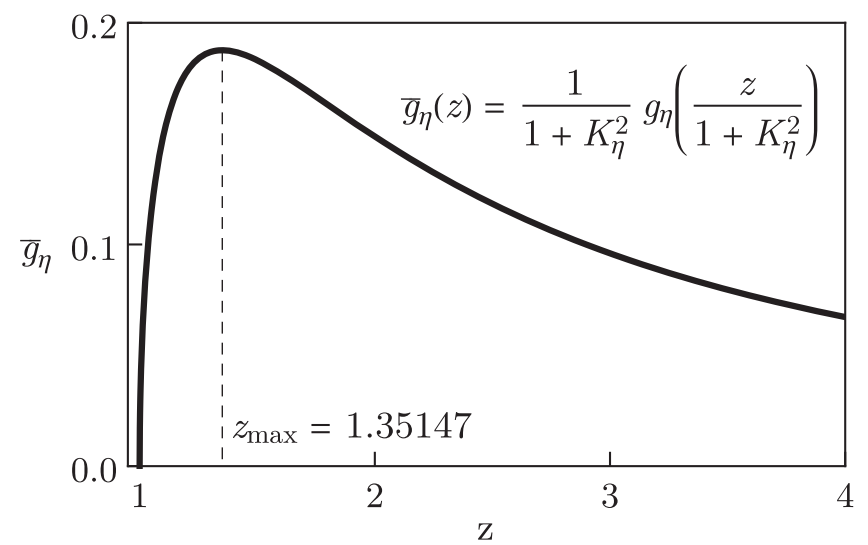

FIG. 19. Plot of $\bar{g}_{\eta}(z)$ given in Eq. (L8) for $K_{\eta}=\sqrt{30}$.

The PDF is plotted in Fig. 16. Its maximum lies very close to the left boundary of its domain $x \in\left[1 /\left(1+K_{\eta}^{2}\right), \infty\right)$. For $x \ll 1$, the PDF $g_{\eta}(x)$ depends on $x$ in terms of the rescaled parameter $z=x\left(1+K_{\eta}^{2}\right)$, whose PDF is

$$
\bar{g}_{\eta}(z) \simeq \frac{\operatorname{erf}\left(\sqrt{\frac{1}{2} \log z}\right)}{z^{3 / 2} \sqrt{2}} .
$$

The plot of $\bar{g}_{\eta}(z)$ is given in Fig. 19, and its maximum $z_{\max } \simeq 1.35$. Typical values of $x_{m} \simeq z_{\max } / K_{\eta}^{2} \ll 1$ correspond to $w_{m} \sim 1$ and to a broad PDF of marked state energies, $\left|z-\epsilon_{m}\right| \sim W$.

We are interested in the limits [cf. Eq. (K12)]

$$
x \gg 1, \quad K_{\eta} \gg 1 .
$$

We note that $\log x \gg|\log y|$ in the denominator of Eq. (L7) for all $y$ except for the small interval

$$
\frac{1}{\sqrt{1+K_{\eta}^{2}}} \leq y \lesssim \frac{1}{x},
$$

whose contribution to the integral is neglected. Expanding the integrand in powers of $(\log x)^{-1 / 2}$, we get

$$
g_{\eta}(x) \simeq \frac{\pi}{2 K_{\eta}} g_{\infty}(x)-\frac{\pi \log 2}{2 K_{\eta} \pi^{1 / 2} x^{2} \log ^{\frac{3}{2}} x},
$$

where function $g_{\infty}(x)$ is defined in Eq. (60). We observe from Eqs. (K10) and (K14) that $\eta\left\langle h_{m}\right\rangle=\left[\pi /\left(2 K_{\eta}\right)\right]$. Using the expressions for $g_{\infty}$ [Eq. (60)] and $\langle h\rangle$ [Eq. (K14)], we obtain under the condition (L9)

$$
g_{\eta}(x) \simeq \eta\langle h\rangle g_{\infty}\left(\frac{x}{\eta\langle h\rangle}\right), \quad x \gg 1 .
$$

Given a large deviation of $x_{m}$ satisfying Eq. (L9), the conditional PDF of $\eta h_{m}$ is narrowly peaked around its 
mean value corresponding to $\left|\epsilon_{m}-z\right| \sim \eta$. In contrast, typical values of $x_{m}$ correspond to a much broader PDF of $\epsilon_{m} \sim W$. This correspondence gives rise to a small factor $\pi / 2 K_{\eta} \sim \eta / W$ in the leading-order term in Eq. (L10).

\section{Characteristic function of the PDF of the elements in the sum}

The relation between the characteristic functions $\phi_{\eta}(u)$ and $\phi_{\infty}(u)$ [Eq. (H1)] in the limit

$$
|u| \ll 1
$$

should be the same as the relation (L10) between the corresponding PDFs $g_{\eta}(x)$ and $g_{\infty}(x)$ in the limit of large $x$ [Eq. (L9)]. Here, we establish this relation directly. We break $\phi_{\eta}(u)$ in two parts

$$
\phi_{\eta}(u)=\phi_{\eta}^{1}(u)+\phi_{\eta}^{2}(u),
$$

where

$$
\begin{gathered}
\phi_{\eta}^{1}(u)=\int_{1 /\left(1+K_{\eta}^{2}\right)}^{1} d w g_{\eta}(w)\left(e^{i u w}-1\right), \\
\phi_{\eta}^{2}(u)=\int_{1}^{\infty} d w g_{\eta}(w)\left(e^{i u w}-1\right) .
\end{gathered}
$$

Expanding $\phi_{\eta}^{1}(u)$ in $u$, we get

$$
\phi_{\eta}^{1}(u) \simeq \frac{\pi}{2 K_{\eta}} i \zeta_{1} u,
$$

where

$$
\zeta_{1}=\frac{2}{\pi^{3 / 2}} \int_{0}^{1} \frac{d x}{x} \int_{0}^{\sqrt{x}} d y \frac{1}{\sqrt{\left(1-y^{2}\right) \log \left(x / y^{2}\right)}} .
$$

To calculate $\phi_{\eta}^{2}(u)$ in the limit of small $|u|$, we introduce $X \gg 1$ such that

$$
|u| \ll X|u| \ll 1
$$

and write

$$
\phi_{\eta}^{2}(u)=\phi_{\eta}^{2,-}(u)+\phi_{\eta}^{2,+}(u) .
$$

Here,

$$
\begin{aligned}
& \phi_{\eta}^{2,-}(u)=\int_{1}^{x} d x g_{\eta}(x)\left(e^{i u x}-1\right), \\
& \phi_{\eta}^{2,+}(u)=\int_{X}^{\infty} d x g_{\eta}(x)\left(e^{i u x}-1\right) .
\end{aligned}
$$

We use Eq. (L18) and expand $\phi_{\eta}^{2,-}(u)$ in $u$ :

$$
\phi_{\eta}^{2,-}(u) \simeq i u \int_{1}^{x} g_{\eta}(x) d x
$$

To calculate the term $\phi_{K}^{2,+}(u)$, we use the approximation (L10) and write

$$
\begin{array}{r}
\phi_{\eta}^{2,+}(u)=\frac{\pi}{2 K_{\eta}} \phi_{\infty}(u)-i u \frac{\pi}{2 K_{\eta}} \int_{1}^{X} g_{\infty}(x) x d x \\
-\frac{\pi \log 2}{2 K_{\eta}} \int_{X}^{\infty} d x \frac{e^{i u x}-1}{\sqrt{\pi} x^{2}(\log x)^{3 / 2}},
\end{array}
$$

where the characteristic function $\phi_{\infty}$ is defined in Eq. (H1).

Combining $\phi_{\eta}^{2, \pm}(u)$ together and taking the limit $X \rightarrow \infty$, we get after some transformations

$$
\begin{aligned}
\phi_{\eta}^{2}(u) & \simeq \frac{\pi}{2 K_{\eta}}\left[\phi_{\infty}(u)-i \zeta_{2} u\right], \\
\zeta_{2} & =\left(\frac{32}{\pi^{3}}\right)^{1 / 2} \int_{0}^{1} d y\left(\frac{\log (1 / y)}{1-y^{2}}\right)^{1 / 2} .
\end{aligned}
$$

After some transformations, one can show that $\zeta_{1}=\zeta_{2}$. Therefore, terms $\sim u$ in $\phi_{\eta}^{1}(u)$ and $\phi_{\eta}^{2}(u)$ cancel each other. Combining these two quantities together in Eq. (L13), we finally get

$$
\phi_{\eta}(u) \simeq \frac{\pi}{2 K_{\eta}} \phi_{\infty}(u)+\mathcal{O}\left(\frac{|u|}{K_{\eta}|\log u|^{3 / 2}}\right) .
$$

As expected, this relation corresponds to the relation (L10) between the PDFs $g_{k}$ and $g_{\infty}$.

\section{GCLT for the sum}

We now revisit the expression (L5) for the PDF of the variable $\Sigma^{\prime \prime}$ [Eq. (L1)]. In the limit $M \rightarrow \infty$, the integral over $k$ in the rhs of Eq. (L5) is dominated by small values of the argument in $\phi_{\eta}\left(k V_{\mathrm{typ}}^{2} / \eta\right)$. Then, using Eqs. (L26) and (K10), we get after the change of a variable of integration in Eq. (L5)

$$
\operatorname{PDF}\left(\Sigma^{\prime \prime}\right)=\frac{1}{2 \pi \Sigma_{*}^{\prime \prime}} \int_{-\infty}^{\infty} d q e^{-i q\left(\Sigma^{\prime \prime} / \Sigma_{*}^{\prime \prime}\right)+\Omega_{\eta} \phi_{\infty}\left(q / \Omega_{\eta}\right),}
$$

where $\Sigma_{*}^{\prime \prime}$ [Eq. (92) [is the characteristic value of the imaginary part of the self-energy of marked states obtained in the FGR-based calculation in Sec. VIII, and the quantity $\Omega_{\eta}$ equals

$$
\Omega_{\eta}=\frac{\pi M}{2 K_{\eta}}=\frac{\pi \eta}{\delta \epsilon}
$$

It has a meaning of the typical number of marked states within the nonergodic miniband of the width $\eta$ [cf. Eq. (98) and Fig. 14]. 
We make a self-consistent assumption [cf. Eq. (134) in the main text] and set

$$
\eta=\Sigma_{*}^{\prime \prime}
$$

Then, one can immediately see that

$$
\Omega_{\eta}=\Omega_{\Sigma_{*}^{\prime \prime}}=\Omega,
$$

where $\Omega$ is the typical number of marked states in a miniband defined in Eq. (113).

Comparing the expression (L27) with Eqs. (I4) and (L30), we represent the random variable $\Sigma^{\prime \prime}$ in the form

$$
\Sigma^{\prime \prime} \stackrel{d}{=} \sigma_{\Omega} \Sigma_{*}^{\prime \prime} x+b_{\Omega} \Sigma_{*}^{\prime \prime}, \quad \operatorname{PDF}(x)=L_{1}^{1,1}(x) .
$$

Here, random variable $x$ obeys a Levy alpha-stable distribution [Eq. (I8)] shown in Fig. 15. The quantities $b_{\Omega}$ and $\sigma_{\Omega}$ are given as

$$
\begin{gathered}
\sigma_{\Omega}=\sqrt{\frac{\pi}{4 \log \Omega}}, \\
b_{\Omega} \simeq \sigma_{\Omega}^{-1}-\frac{2}{\pi} \sigma_{\Omega} \log \left(\sigma_{\Omega}^{-1}\right)+\frac{2}{\pi}\left(1-\gamma_{\text {Euler }}\right) \sigma_{\Omega} .
\end{gathered}
$$

Their dependence on $\Omega$ is given in the main text [Eqs. (96) and (95)], where we should replace $M$ with $\Omega$.

\section{APPENDIX M: NUMERICAL SIMULATIONS}

In this section, we provide details of the numerical analysis of the ensemble of Hamiltonians introduced in Sec. VI in addition to the results in Sec. VII.

\section{Numerical justification of cavity equations}

The application of the cavity method to the case of the ensemble of dense matrices considered in this paper (see Sec. VI) exploits the similarity between the local structure of the adjacency graph of the Hamiltonian $\mathcal{H}$ and the Bethe lattice. The derivation of the cavity equations (102a) and (102b) for the case of $\mathcal{H}$ outlined in Sec. X neglects off-diagonal terms $Y$ in comparison to diagonal $X$, which is justified for graphs with an extensive number of neighbors [33], where

$$
\begin{gathered}
X=\frac{1}{M} \sum_{j} \mathcal{H}_{1 j}^{2} G_{j j}(z), \\
Y=\frac{2}{M} \sum_{j \neq k} \mathcal{H}_{1 j} \mathcal{H}_{1 k} G_{j k}(z),
\end{gathered}
$$

where $G_{i j}$ is the single-particle Green function corresponding to the Hamiltonian $\mathcal{H}$ at an energy near the center of the band, introduced in Sec. X. It has been shown for Levy

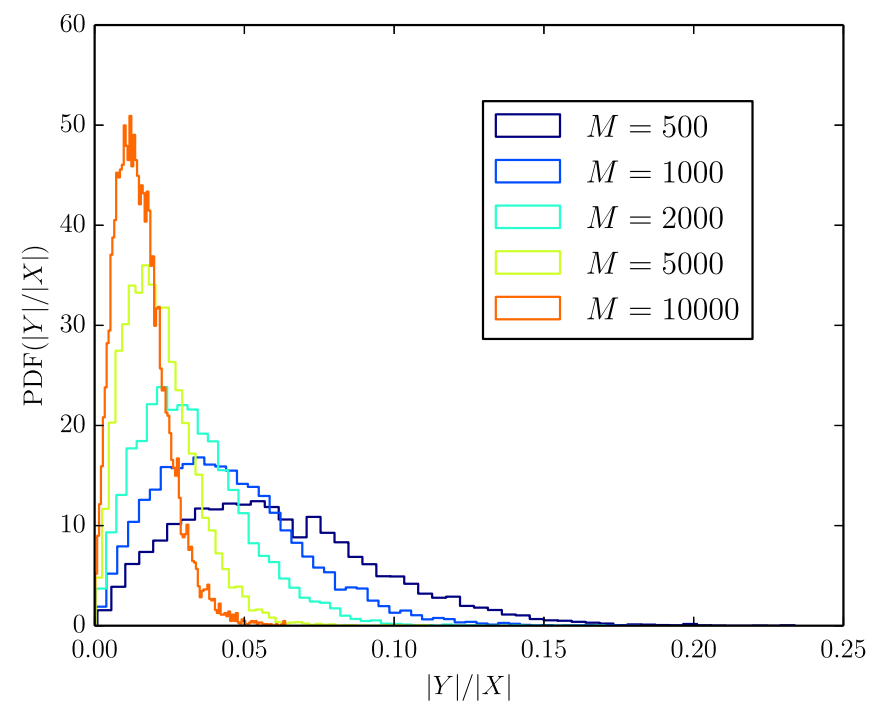

FIG. 20. Probability distribution of the ratio $|Y / X|$ defined in Eqs. (M1) and (M2) for $\gamma=0.6$.

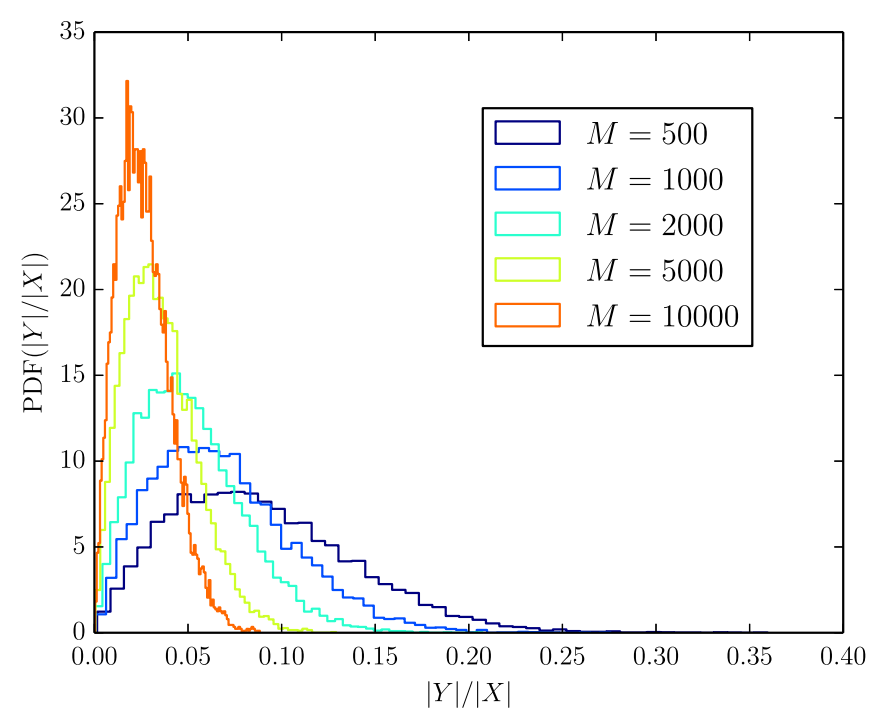

FIG. 21. The same as in Fig. 20 but with $\gamma=1.2$.

matrices [33] that the ratio $|Y / X|$ scales to zero with growing matrix size $M$ and, therefore, can be neglected. This argument could be extended to PBLMs considered in this paper. We confirm the validity of this approximation numerically by analyzing the probability distribution of the ratio $|Y / X|$ as a function of the matrix size $M$. In Figs. 20-22, the distribution of $|Y / X|$ scales towards a high weight at vanishing values of $|Y / X|$ with growing $M$.

\section{Numerical analysis of population transfer time \\ a. Population transfer time from the dynamical correlator}

In addition to Fig. 13 in Sec. VII of the main text, we perform a similar collapse of the dynamical correlator 


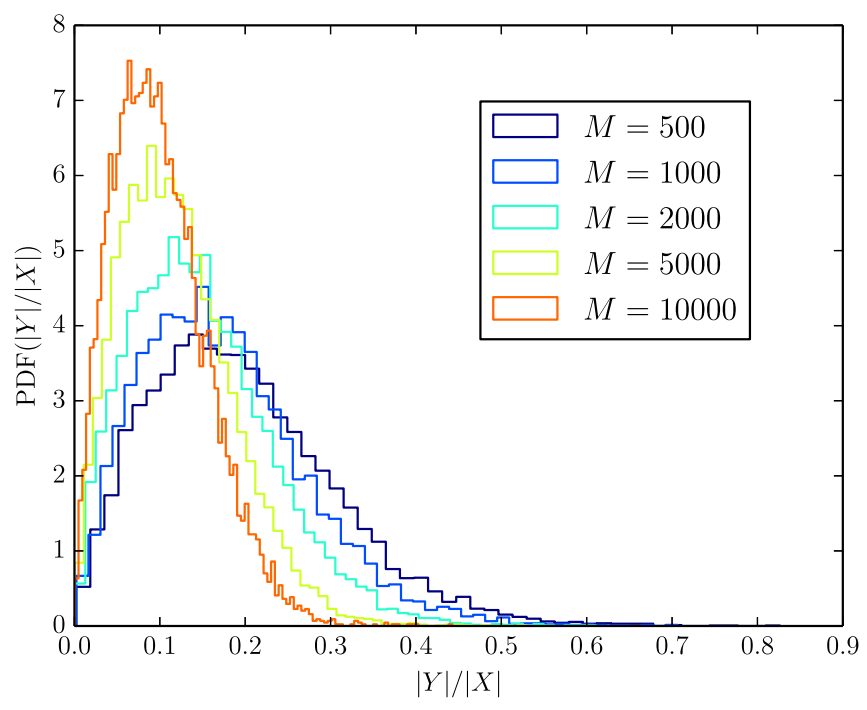

FIG. 22. The same as in Fig. 20 but for $\gamma=1.6$.

frequency dependence for different matrix sizes $M$ for a range of different values of $\gamma$. In Figs. 23-26, the characteristic energy scale extracted from each set of plots using this procedure $\Gamma_{\varepsilon}=\Gamma_{\text {typ }} M^{\varepsilon}$ corresponds to the typical miniband width with the respective value of the parameter $\gamma$. The fitting parameter in the scaling exponent $\varepsilon$ is small for all $\gamma$ we consider and is consistent with the finite size effect.

\section{b. Population transfer probability as a function of time}

In the main text, we analyze the complexity of the PT protocol using the solution of the full nonlinear cavity equations for the size of the typical miniband and estimate the number of states in the miniband using the classical value of the level spacing $W / M$. In this section, we analyze the scaling of the population transfer time using exact

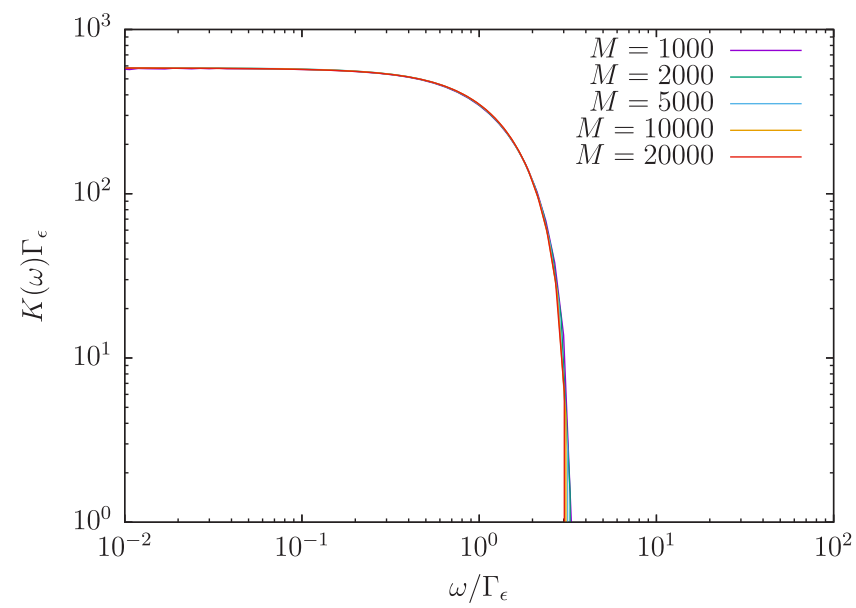

FIG. 23. $K(\omega)$ rescaled with the characteristic energy $\Gamma_{\varepsilon}=$ $2 \Sigma_{\text {typ }}^{\prime \prime} M^{\varepsilon}$ where the typical miniband width is given by Eq. (128). Here, $\gamma=1$ with fitting exponent $\varepsilon=-0.025$.

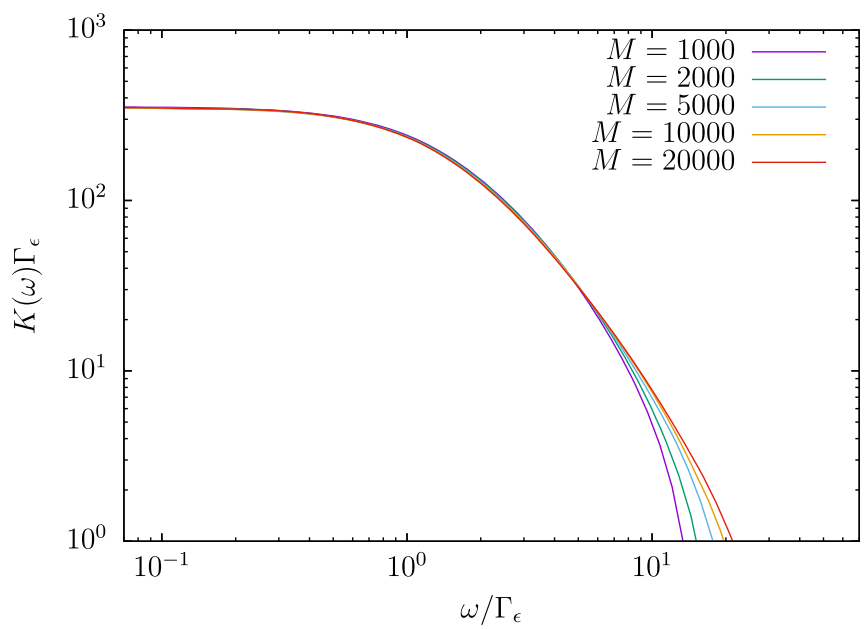

FIG. 24. The same as in Fig. 23 but with $\gamma=1.4$ and fitting exponent $\varepsilon=0.04$.

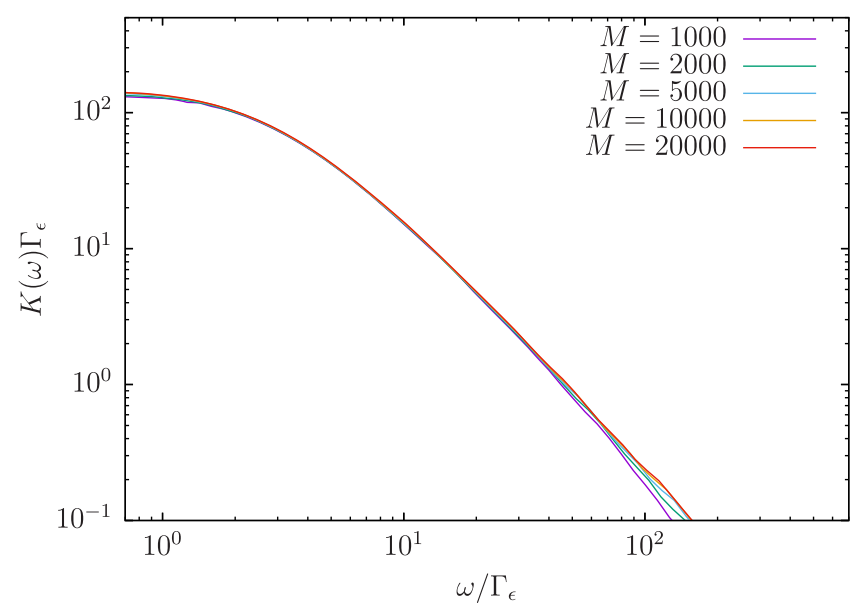

FIG. 25. The same as in Fig. 23 but with $\gamma=1.8$ and fitting exponent $\varepsilon=-0.05$.

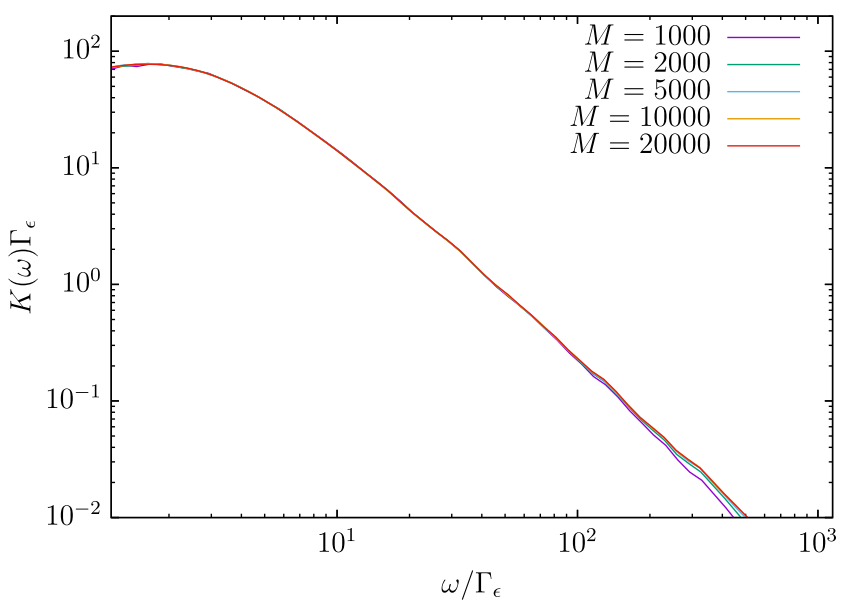

FIG. 26. The same as in Fig. 23 but with $\gamma=2$ and fitting exponent $\varepsilon=-0.055$. 


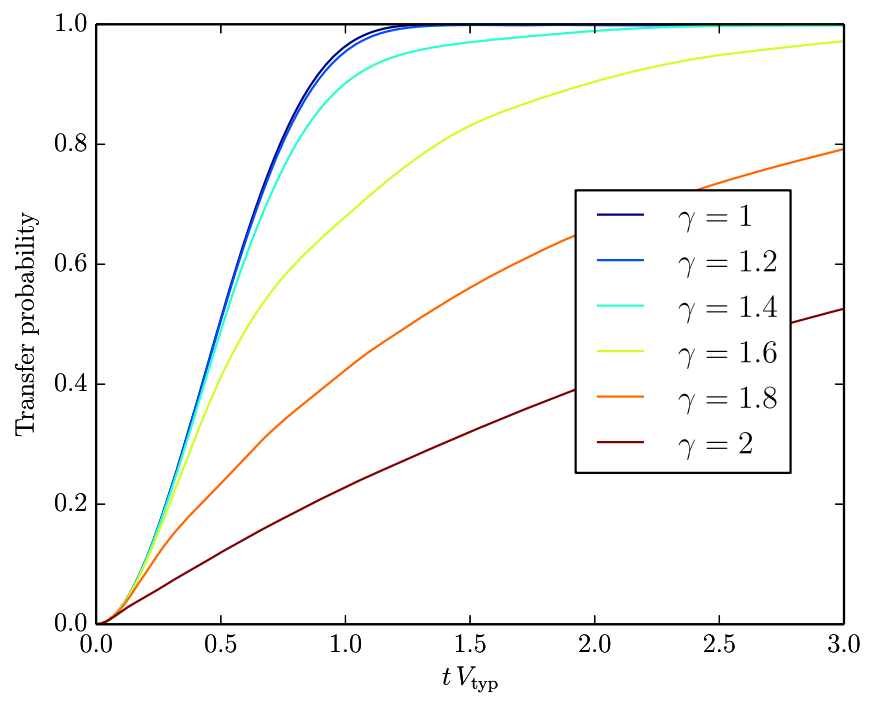

FIG. 27. Population transfer probability as a function of time $t$ in units of $1 / V_{\text {typ }}$ for various values of parameter $\gamma=2 a$.

numerical time evolution. We contrast the population transfer time obtained from the characteristic energy scale of the frequency dependence of the dynamical correlator in Figs. 23-26 with the time dependence of the transfer probability:

$$
p(t)=|\langle i \mid \psi(t)\rangle|^{2},
$$

where $|i\rangle$ is the initial bit string and $|\psi(t)\rangle$ is the wave function resulting from the evolution with the impurity band Hamiltonian in transverse field $\mathcal{H}$ (see Sec. VI) for a time $t$, which is the quantity directly observed experimentally. Note that in Fig. 27 the timescale at which the transfer

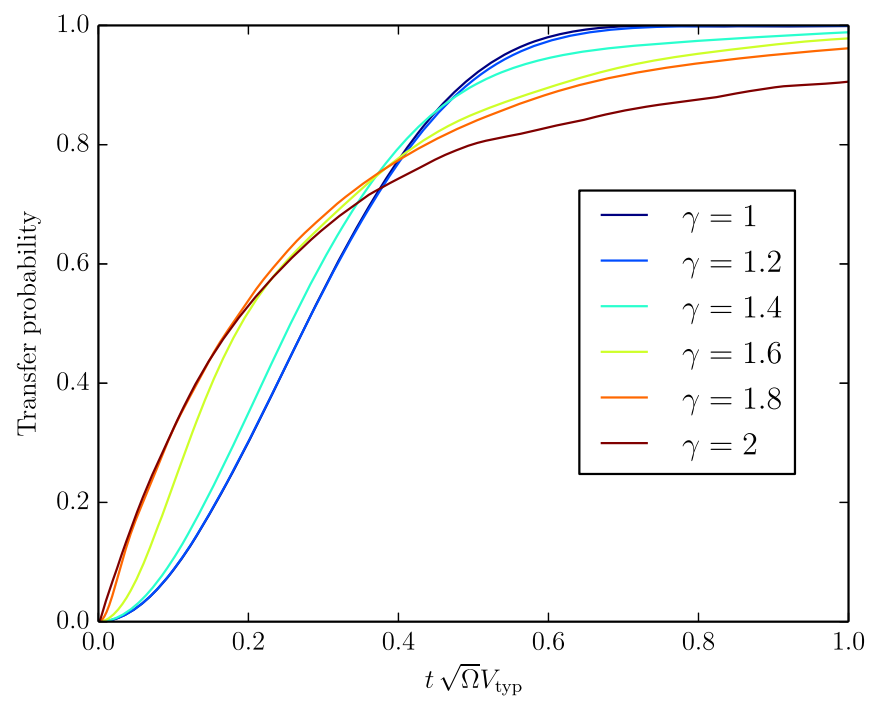

FIG. 28. Population transfer probability as a function of time rescaled with the effective miniband width $\sqrt{\Omega}$ where the number of states in the miniband is estimated using Fermi's golden rule $\Omega=M^{2-\gamma}$; see Eq. (100) of the main text. probability becomes of the order of one depends strongly on the parameter $\gamma$, reflecting the fact that the characteristic time is determined by the size of the many-body miniband $\Gamma$ rather than the typical off-diagonal matrix element $V_{\text {typ }}$. To verify this result, in Fig. 28 we rescale the unit of time with the square root of the number of states in the miniband $\sqrt{\Omega}$, a good approximation for the scaling of the miniband; see Sec. VIII for a qualitative discussion and Sec. XI for rigorous results. We observe an approximate collapse of the curves for different values of $\gamma$ corroborating the PT run-time scaling presented in the main text as well as the estimate of the number of states in the miniband.

[1] L. K. Grover, Quantum Mechanics Helps in Searching for a Needle in a Haystack, Phys. Rev. Lett. 79, 325 (1997).

[2] T. Kadowaki and H. Nishimori, Quantum Annealing in the Transverse Ising Model, Phys. Rev. E 58, 5355 (1998).

[3] E. Farhi, J. Goldstone, S. Gutmann, J. Lapan, A. Lundgren, and D. Preda, A Quantum Adiabatic Evolution Algorithm Applied to Random Instances of an $\mathrm{np}$-Complete Problem, Science 292, 472 (2001).

[4] J. Brooke, D. Bitko, G. Aeppli et al., Quantum Annealing of a Disordered Magnet, Science 284, 779 (1999).

[5] V. N. Smelyanskiy, U.v. Toussaint, and D. A. Timucin, Dynamics of Quantum Adiabatic Evolution Algorithm for Number Partitioning, arXiv:quant-ph/0202155.

[6] S. Boixo, T. F. Rønnow, S. V. Isakov, Z. Wang, D. Wecker, D. A. Lidar, J. M. Martinis, and M. Troyer, Evidence for Quantum Annealing with More than One Hundred Qubits, Nat. Phys. 10, 218 (2014).

[7] S. Knysh, Zero-Temperature Quantum Annealing Bottlenecks in the Spin-Glass Phase, Nat. Commun. 7, 12370 (2016).

[8] S. Boixo, V. N. Smelyanskiy, A. Shabani, S. V. Isakov, M. Dykman, V. S. Denchev, M. H. Amin, A. Y. Smirnov, M. Mohseni, and H. Neven, Computational Multiqubit Tunnelling in Programmable Quantum Annealers, Nat. Commun. 7, 10327 (2016).

[9] V. S. Denchev, S. Boixo, S. V. Isakov, N. Ding, R. Babbush, V. Smelyanskiy, J. Martinis, and H. Neven, What Is the Computational Value of Finite-Range Tunneling?, Phys. Rev. X 6, 031015 (2016).

[10] T. Albash and D. A. Lidar, Adiabatic Quantum Computation, Rev. Mod. Phys. 90, 015002 (2018).

[11] E. Farhi, J. Goldstone, and S. Gutmann, A Quantum Approximate Optimization Algorithm, arXiv:1411.4028.

[12] M. Mézard, G. Parisi, and M. Virasoro, Spin Glass Theory and Beyond: An Introduction to the Replica Method and Its Applications (World Scientific, Singapore, 1987), Vol. 9.

[13] Y. Fu and P. W. Anderson, Application of Statistical Mechanics to np-Complete Problems in Combinatorial Optimisation, in Spin Glass Theory and Beyond: An Introduction to the Replica Method and Its Applications (World Scientific, Singapore, 1987), pp. 357-372.

[14] B. Altshuler, H. Krovi, and J. Roland, Anderson Localization Makes Adiabatic Quantum Optimization Fail, Proc. Natl. Acad. Sci. U.S.A. 107, 12446 (2010). 
[15] P. W. Anderson, Absence of Diffusion in Certain Random Lattices, Phys. Rev. 109, 1492 (1958).

[16] D. Basko, I. Aleiner, and B. Altshuler, Metal-Insulator Transition in a Weakly Interacting Many-Electron System with Localized Single-Particle States, Ann. Phys. (Amsterdam) 321, 1126 (2006).

[17] V. Oganesyan and D. A. Huse, Localization of Interacting Fermions at High Temperature, Phys. Rev. B 75, 155111 (2007).

[18] C. R. Laumann, A. Pal, and A. Scardicchio, Many-Body Mobility Edge in a Mean-Field Quantum Spin Glass, Phys. Rev. Lett. 113, 200405 (2014).

[19] G. Mossi and A. Scardicchio, Ergodic and Localized Regions in Quantum Spin Glasses on the Bethe Lattice, Phil. Trans. R. Soc. A 375, 20160424 (2017).

[20] S. Knysh and V. Smelyanskiy, On the Relevance of Avoided Crossings away from Quantum Critical Point to the Complexity of Quantum Adiabatic Algorithm, arXiv: 1005.3011.

[21] V. E. Kravtsov, I. M. Khaymovich, E. Cuevas, and M. Amini, A Random Matrix Model with Localization and Ergodic Transitions, New J. Phys. 17, 122002 (2015).

[22] B. L. Altshuler, E. Cuevas, L. B. Ioffe, and V. E. Kravtsov, Nonergodic Phases in Strongly Disordered Random Regular Graphs, Phys. Rev. Lett. 117, 156601 (2016).

[23] K.S. Tikhonov and A. D. Mirlin, Fractality of Wave Functions on a Cayley Tree: Difference between Tree and Locally Treelike Graph without Boundary, Phys. Rev. B 94, 184203 (2016).

[24] K. S. Tikhonov, A. D. Mirlin, and M. A. Skvortsov, Anderson Localization and Ergodicity on Random Regular Graphs, Phys. Rev. B 94, 220203(R) (2016).

[25] M. Sonner, K. S. Tikhonov, and A. D. Mirlin, Multifractality of Wave Functions on a Cayley Tree: From Root to Leaves, Phys. Rev. B 96, 214204 (2017).

[26] Recently, the FSA was used to analyze quantum spin-glass dynamics [18,27-29]. This approach relies on the small parameter $B_{\perp} \ll 1$ such that the matrix element between bit strings separated by the Hamming distance (number of spin flips) $d$ is given by the leading-order perturbation theory $\Delta(d) \propto d !\left(B_{\perp}\right)^{d}$, the matrix element of a single spin flip times the number of trajectories connecting the two bit strings at distance $d$ !. Note that $\Delta(d)$ is growing with $B_{\perp}$. This dependence cannot proceed to arbitrary large field $B_{\perp} \gtrsim 1$. In the class of models characterized by uncorrelated energies such as the IB in Eq. (3) or the random energy model [30], there is a natural limit for the growth of the matrix element. A tunneling matrix element between two typical bit strings, which are separated by Hamming distance $d=n / 2$ with high probability, proceeds via ergodic virtual states near the maximum of the density of states which are independent random unitary vectors, in which case $\Delta(n / 2) \sim \sum_{\psi}\langle z \mid \psi\rangle\left\langle\psi \mid z^{\prime}\right\rangle \sim 2^{-n / 2}$, where $\psi$ are independent random vectors. This limit clearly cannot be obtained from the strictly growing FSA expression for $\Delta(d)$. Moreover, as we show below, even at small $B_{\perp} \ll 1$, the asymptotically exact expression for the matrix element differs from the FSA by an exponential factor; see Eq. (24) in the main text.
[27] C. L. Baldwin, C. R. Laumann, A. Pal, and A. Scardicchio, The Many-Body Localized Phase of the Quantum Random Energy Model, Phys. Rev. B 93, 024202 (2016).

[28] C. L. Baldwin, C. R. Laumann, A. Pal, and A. Scardicchio, Clustering of Nonergodic Eigenstates in Quantum Spin Glasses, Phys. Rev. Lett. 118, 127201 (2017).

[29] C. L. Baldwin and C. R. Laumann, Quantum Algorithm for Energy Matching in Hard Optimization Problems, Phys. Rev. B 97, 224201 (2018).

[30] B. Derrida, Random-Energy Model: An Exactly Solvable Model of Disordered Systems, Phys. Rev. B 24, 2613 (1981).

[31] B. I. Shklovskii and A. L. Efros, Electronic Properties of Doped Semiconductors (Springer Science, New York, 2013), Vol. 45.

[32] R. Abou-Chacra, D. Thouless, and P. Anderson, A SelfConsistent Theory of Localization, J. Phys. C 6, 1734 (1973).

[33] P. Cizeau and J.-P. Bouchaud, Theory of Lévy Matrices, Phys. Rev. E 50, 1810 (1994).

[34] F. L. Metz, I. Neri, and D. Bollé, Localization Transition in Symmetric Random Matrices, Phys. Rev. E 82, 031135 (2010).

[35] E. Tarquini, G. Biroli, and M. Tarzia, Level Statistics and Localization Transitions of Levy Matrices, Phys. Rev. Lett. 116, 010601 (2016).

[36] D. Facoetti, P. Vivo, and G. Biroli, From Non-Ergodic Eigenvectors to Local Resolvent Statistics and Back: A Random Matrix Perspective, Europhys. Lett. 115, 47003 (2016).

[37] C. Monthus, Localization Transition in Random Lévy Matrices: Multifractality of Eigenvectors in the Localized Phase and at Criticality, J. Stat. Mech. (2016) 093304.

[38] D. Sherrington and S. Kirkpatrick, Solvable Model of a Spin-Glass, Phys. Rev. Lett. 35, 1792 (1975).

[39] T. R. Kirkpatrick and D. Thirumalai, p-Spin-Interaction Spin-Glass Models: Connections with the Structural Glass Problem, Phys. Rev. B 36, 5388 (1987).

[40] M. Mézard, G. Parisi, and R. Zecchina, Analytic and Algorithmic Solution of Random Satisfiability Problems, Science 297, 812 (2002).

[41] G. E. Santoro, R. Martonak, E. Tosatti, and R. Car, Theory of Quantum Annealing of an Ising Spin Glass, Science 295, 2427 (2002).

[42] B. Heim, T. F. Rønnow, S. V. Isakov, and M. Troyer, Quantum versus Classical Annealing of Ising Spin Glasses, Science 348, 215 (2015).

[43] S. V. Isakov, G. Mazzola, V. N. Smelyanskiy, Z. Jiang, S. Boixo, H. Neven, and M. Troyer, Understanding Quantum Tunneling through Quantum Monte Carlo Simulations, Phys. Rev. Lett. 117, 180402 (2016).

[44] Z. Jiang, V. N. Smelyanskiy, S. V. Isakov, S. Boixo, G. Mazzola, M. Troyer, and H. Neven, Scaling Analysis and Instantons for Thermally Assisted Tunneling and Quantum Monte Carlo Simulations, Phys. Rev. A 95, 012322 (2017).

[45] K. Fisher and J. Hertz, Spin Glasses (Cambridge University Press, Cambridge, England, 1991).

[46] E. Farhi and S. Gutmann, Analog Analogue of a Digital Quantum Computation, Phys. Rev. A 57, 2403 (1998). 
[47] H. Neven, Enhancing Simulated Annealing with Quantum Annealing, U.S. Patent Application Publication No. PCT/ US2016/068 400, 2015, https://patents.google.com/patent/ WO2017117016A1.

[48] N. Chancellor, Modernizing Quantum Annealing Using Local Searches, New J. Phys. 19, 023024 (2017).

[49] B. Altshuler, L. Ioffe, and V. Kravtsov, Multifractal States in Self-Consistent Theory of Localization: Analytical Solution, arXiv:1610.00758.

[50] The same plateau width characterizes the frequency dependence of the eigenfunction overlap correlation coefficient $K(\omega)=M \sum_{j=1}^{M} \sum_{\beta, \beta^{\prime}}\left|\left\langle j \mid \psi_{\beta}\right\rangle\right|^{2}\left|\left\langle j \mid \psi_{\beta^{\prime}}\right\rangle\right|^{2} \delta\left(\omega-E_{\beta}+E_{\beta^{\prime}}\right)[21]$.

[51] N. Rosenzweig and C. E. Porter, "Repulsion of Energy Levels” in Complex Atomic Spectra, Phys. Rev. 120, 1698 (1960).

[52] Z. Burda, J. Jurkiewicz, M. A. Nowak, G. Papp, and I. Zahed, Free Random Lévy and Wigner-Lévy Matrices, Phys. Rev. E 75, 051126 (2007).

[53] B. Gnedenko and A. Kolmogorov, Limit Distributions for Sums of Independent (Addison-Wesley, 1954).

[54] A. Garg, Application of the Discrete Wentzel-KramersBrillouin Method to Spin Tunneling, J. Math. Phys. (N.Y.) 39, 5166 (1998).

[55] A. Garg and M. Stone, Bohr-Sommerfeld Quantization of Spin Hamiltonians, Phys. Rev. Lett. 92, 010401 (2004).

[56] M. Novaes and M. A. M. de Aguiar, Semiclassical Husimi Functions for Spin Systems, Phys. Rev. A 71, 012104 (2005).

[57] P. Braun, Discrete Semiclassical Methods in the Theory of Rydberg Atoms in External Fields, Rev. Mod. Phys. 65, 115 (1993).

[58] R. S. Varga, Geř̌gorin and His Circles (Springer Science, New York, 2010), Vol. 36.

[59] A. De Luca, A. Scardicchio, V. E. Kravtsov, and B. L. Altshuler, Support Set of Random Wave-Functions on the Bethe Lattice, arXiv:1401.0019.

[60] G. D. Mahan, Many-Particle Physics (Springer Science, New York, 2013).

[61] Wikipedia, Stable Distribution, https://en.wikipedia.org/ wiki/Stable_distribution.

[62] J. Voit, The Statistical Mechanics of Financial Markets (Springer Science, New York, 2013).
[63] T. Rogers, I. P. Castillo, R. Kühn, and K. Takeda, Cavity Approach to the Spectral Density of Sparse Symmetric Random Matrices, Phys. Rev. E 78, 031116 (2008).

[64] T. Tao, Topics in Random Matrix Theory (American Mathematical Society, Providence, RI, 2012), Vol. 132.

[65] E. Farhi, J. Goldstone, S. Gutmann, and M. Sipser, Quantum Computation by Adiabatic Evolution, arXiv:quant-ph/ 0001106.

[66] A. M. Childs, E. Deotto, E. Farhi, J. Goldstone, S. Gutmann, and A. J. Landahl, Quantum Search by Measurement, Phys. Rev. A 66, 032314 (2002).

[67] G.-L. Long, X. Li, and Y. Sun, Phase Matching Condition for Quantum Search with a Generalized Initial State, Phys. Lett. A 294, 143 (2002).

[68] N. Shenvi, K. R. Brown, and K. B. Whaley, Effects of a Random Noisy Oracle on Search Algorithm Complexity, Phys. Rev. A 68, 052313 (2003).

[69] E. Kogan, On the Analytic Structure of Green's Function for the Fano-Anderson Model, arXiv:quant-ph/0611043.

[70] A. M. Childs and J. Goldstone, Spatial Search by Quantum Walk, Phys. Rev. A 70, 022314 (2004).

[71] Y. Y. Goldschmidt, Solvable Model of the Quantum Spin Glass in a Transverse Field, Phys. Rev. B 41, 4858 (1990).

[72] V. N. Smelyanskiy, K. Kechedzhi, S. Boixo, H. Neven, and B. Altshuler, Intermittency of Dynamical Phases in a Quantum Spin Glass, arXiv:1907.01609.

[73] L. Faoro, M. V. Feigelman, and L. Ioffe, Non-Ergodic Extended Phase of the Quantum Random Energy Model, Ann. Phys. (Amsterdam) 409, 167916 (2019).

[74] The expression (B8) for $m_{0}(E)$ should be evaluated for the energy $E=E^{0)} \simeq-n-B_{\perp}^{2}$ corresponding to the eigenvalues of impurity band [Eqs. (36) and (38)]. In this case, the $\mathcal{O}\left(n^{0}\right)$ corrections in the rhs of Eq. (B8) vanish:

$$
m_{0}\left(E^{(0)}\right) \simeq \frac{n}{2} \sqrt{1-B_{\perp}^{-2}}+\frac{1-3 B_{\perp}^{2}}{4 n \sqrt{1-B_{\perp}^{-2}}} .
$$

The terms $\mathcal{O}(1 / n)$ above can be neglected in the exponents of the WKB solutions (B10) and (B13). 